\title{
ON THE UNIFORM COMPUTATIONAL CONTENT OF RAMSEY'S THEOREM
}

\author{
VASCO BRATTKA AND TAHINA RAKOTONIAINA
}

\begin{abstract}
We study the uniform computational content of Ramsey's theorem in the Weihrauch lattice. Our central results provide information on how Ramsey's theorem behaves under product, parallelization and jumps. From these results we can derive a number of important properties of Ramsey's theorem. For one, the parallelization of Ramsey's theorem for cardinality $n \geq 1$ and an arbitrary finite number of colors $k \geq 2$ is equivalent to the $n$-th jump of weak König's lemma. In particular, Ramsey's theorem for cardinality $n \geq 1$ is $\boldsymbol{\Sigma}_{n+2}^{0}-$ measurable in the effective Borel hierarchy, but not $\boldsymbol{\Sigma}_{n+1}^{0}$-measurable. Secondly, we obtain interesting lower bounds, for instance the $n$-th jump of weak König's lemma is Weihrauch reducible to (the stable version of) Ramsey's theorem of cardinality $n+2$ for $n \geq 2$. We prove that with strictly increasing numbers of colors Ramsey's theorem forms a strictly increasing chain in the Weihrauch lattice. Our study of jumps also shows that certain uniform variants of Ramsey's theorem that are indistinguishable from a non-uniform perspective play an important role. For instance, the colored version of Ramsey's theorem explicitly includes the color of the homogeneous set as output information, and the jump of this problem (but not the uncolored variant) is equivalent to the stable version of Ramsey's theorem of the next greater cardinality. Finally, we briefly discuss the particular case of Ramsey's theorem for pairs, and we provide some new separation techniques for problems that involve jumps in this context. In particular, we study uniform results regarding the relation of boundedness and induction problems to Ramsey's theorem, and we show that there are some significant differences with the nonuniform situation in reverse mathematics.
\end{abstract}

Keywords: computable analysis, Weihrauch lattice, Ramsey's theorem.

\section{Contents}

1. Introduction

2. Preliminaries

3. The Uniform Scenario, Lower Bounds and Products

4. Jumps, Increasing Cardinality and Color and Upper Bounds

5. Ramsey's Theorem for Pairs

6. Separation Techniques for Jumps

7. Boundedness and Induction

8. Conclusion

9. Acknowledgments

References

Date: June 21, 2017. 


\section{INTRODUCTION}

In this paper we study uniform computational properties of Ramsey's theorem for cardinality $n$ and $k$ colors. We briefly recall some basic definitions.

By $[M]^{n}:=\{A \subseteq M:|A|=n\}$ we denote the set of subsets of $M$ with exactly $n$ elements. We identify $k$ with the set $\{0, \ldots, k-1\}$ for every $k \in \mathbb{N}$. We also allow the case $k=\mathbb{N}$. Any map $c:[\mathbb{N}]^{n} \rightarrow k$ with finite range is called a coloring $\left(\right.$ of $\left.[\mathbb{N}]^{n}\right)$. A subset $M \subseteq \mathbb{N}$ is called homogeneous (for $c$ ) if there is some $i \in k$ such that $c(A)=i$ for every $A \in[M]^{n}$. In this situation we write $c(M)=i$, which is understood to imply that $M$ is homogeneous. Frank P. Ramsey proved the following theorem [35].

Theorem 1.1 (Ramsey's theorem $1930[35])$. For every coloring $c:[\mathbb{N}]^{n} \rightarrow k$ with $n, k \geq 1$ there exists an infinite homogeneous set $M \subseteq \mathbb{N}$.

We will abbreviate Ramsey's theorem for cardinality $n$ and $k$ colors by $\mathrm{RT}_{n, k} 1$ The computability theoretic study of Ramsey's theorem started when Specker proved that there exists a computable counterexample for Ramsey's theorem for pairs [38, which shows that Ramsey's theorem cannot be proved constructively.

Theorem 1.2 (Specker $1969[38]$ ). There exists a computable coloring $c:[\mathbb{N}]^{2} \rightarrow k$ without a computable infinite homogeneous set $M \subseteq \mathbb{N}$.

Jockusch provided a very simple proof of Specker's theorem, and he improved Specker's result by showing the following [26].

Theorem 1.3 (Jockusch $1972[26]$ ). For every computable coloring $c:\left[\mathbb{N}^{n} \rightarrow 2\right.$ with $n \geq 1$ there exists an infinite homogeneous set $M \subseteq \mathbb{N}$ such that $M^{\prime} \leq_{\mathrm{T}} \emptyset^{(n)}$. However, there exists a computable coloring $c:[\mathbb{N}]^{n} \rightarrow 2$ for each $n \geq 2$ without an infinite homogeneous set $M \subseteq \mathbb{N}$ that is computable in $\emptyset^{(n-1)}$.

Another cornerstone in the study of Ramsey's theorem was the cone avoidance theorem (Theorem [36. Theorem 2.1]) that was originally proved by Seetapun.

Theorem 1.4 (Seetapun $1995[36]$ ). Let $c:[\mathbb{N}]^{2} \rightarrow 2$ be a coloring that is computable in $B \subseteq \mathbb{N}$, and let $\left(C_{i}\right)_{i}$ be a sequence of sets $C_{i} \subseteq \mathbb{N}$ such that $C_{i} \not_{\mathrm{T}} B$ for all $i \in \mathbb{N}$. Then there exists an infinite homogeneous set $M$ for $c$ such that $C_{i} \mathbb{Z}_{\mathrm{T}} M$ for all $i \in \mathbb{N}$.

This theorem was generalized by Cholak, Jockusch and Slaman who proved in particular the following version [12, Theorem 12.2].

Theorem 1.5 (Cholak, Jockusch and Slaman 2001 [12]). For every computable coloring $c:[\mathbb{N}]^{n} \rightarrow k$ there exists an infinite homogeneous set $M \subseteq \mathbb{N}$ such that $\emptyset^{(n)} \mathbb{Z}_{\mathrm{T}} M^{\prime}$.

Cholak, Jockusch and Slaman also improved Jockusch's theorem (Theorem 1.3) for the case of Ramsey's theorem for pairs [12, 13].

Theorem 1.6 (Cholak, Jockusch and Slaman 2001 [12]). For every computable coloring $c:[\mathbb{N}]^{2} \rightarrow 2$ there exists an infinite homogeneous set $M \subseteq \mathbb{N}$, which is low , i.e., such that $M^{\prime \prime} \leq_{\mathrm{T}} \emptyset^{\prime \prime}$.

We will make use of these and other earlier results in our uniform study of Ramsey's theorem. A substantial number of results in this article are based on the second author's PhD thesis 34. The first uniform results on Ramsey's theorem were published by Dorais, Dzhafarov, Hirst, Mileti and Shafer [16] and by Dzhafarov [17, 18. Among other things they proved the following squashing theorem [16, Theorem 2.5]

\footnotetext{
${ }^{1}$ We do not use the more common abbreviation $\mathrm{RT}_{k}^{n}$ since we will use upper indices to indicate the number of jumps or products.
} 


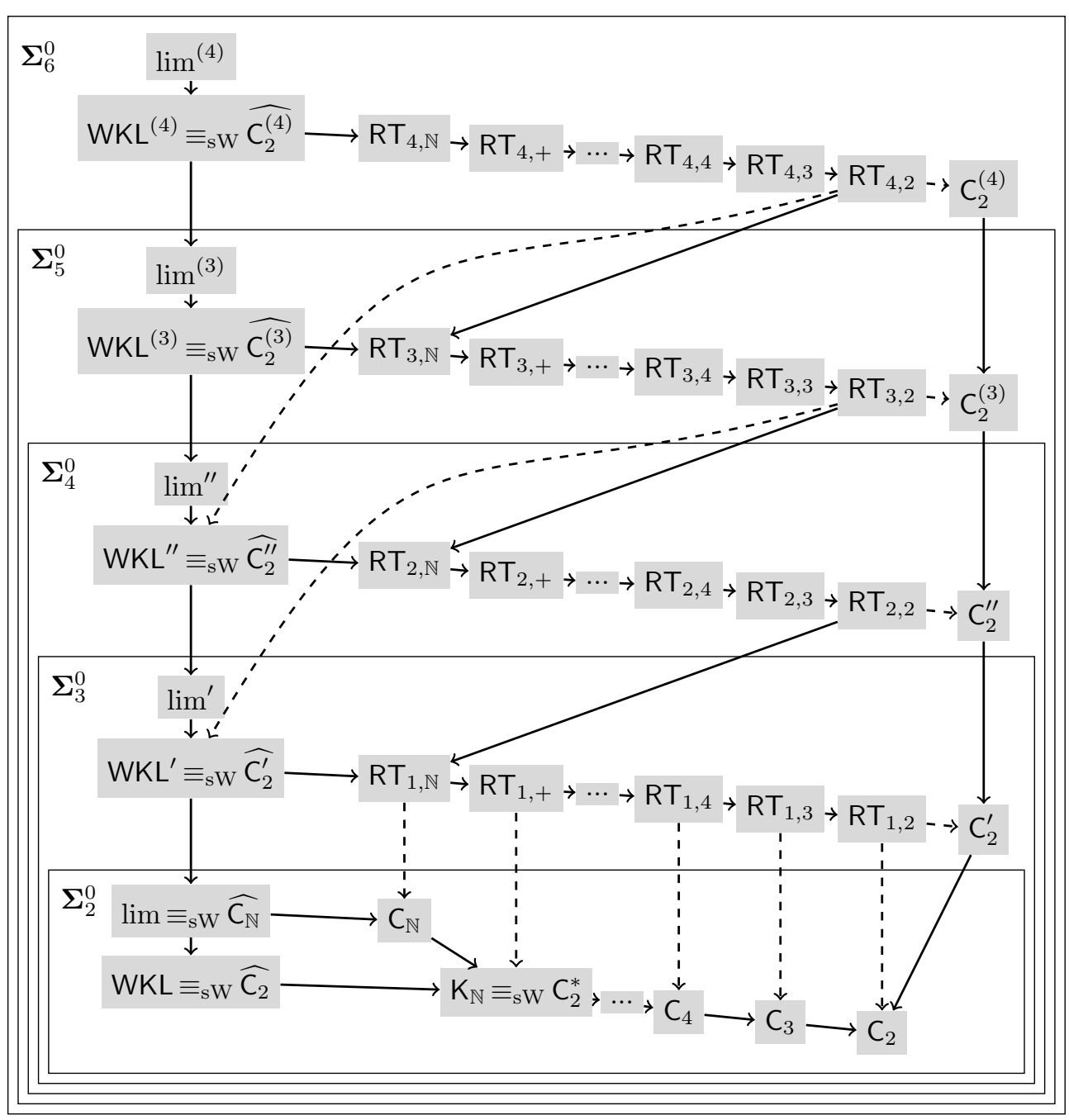

Figure 1. Ramsey's theorem for different cardinalities and colors in the Weihrauch lattice: all solid arrows indicate strong Weihrauch reductions against the direction of the arrow, all dashed arrows indicate ordinary Weihrauch reductions.

that establishes a relation between products and parallelization for problems such as Ramsey's theorem.

Here a problem $f: \subseteq \mathbb{N}^{\mathbb{N}} \rightrightarrows \mathbb{N}^{\mathbb{N}}$, i.e., a partial multi-valued function, is called finitely tolerant if there is a computable partial function $T: \subseteq \mathbb{N}^{\mathbb{N}} \rightarrow \mathbb{N}^{\mathbb{N}}$ such that for all $p, q \in \operatorname{dom}(f)$ and $k \in \mathbb{N}$ with $(\forall n \geq k)(p(n)=q(n))$ it follows that $r \in f(q)$ implies $T\langle r, k\rangle \in f(p)$. By $\langle$,$\rangle we denote a standard pairing function on Baire space$ $\mathbb{N}^{\mathbb{N}}$. Intuitively, finite tolerance means that for two almost identical inputs and a solution for one of these inputs we can compute a solution for the other input. The squashing theorem relates products $g \times f$ to parallelizations $\widehat{g}$ of problems (these notions will be defined in the next section).

Theorem 1.7 (Squashing theorem [16]). For $f, g: \subseteq \mathbb{N}^{\mathbb{N}} \rightrightarrows \mathbb{N}^{\mathbb{N}}$ we obtain:

(1) If $\operatorname{dom}(f)=\mathbb{N}^{\mathbb{N}}$ and $f$ is finitely tolerant, then $g \times f \leq_{\mathrm{W}} f \Longrightarrow \widehat{g} \leq_{\mathrm{W}} f$.

(2) If $\operatorname{dom}(f)=2^{\mathbb{N}}$ and $f$ is finitely tolerant, then $g \times f \leq_{\mathrm{sW}} f \Longrightarrow \widehat{g} \leq_{\mathrm{sW}} f$. 
This theorem allowed the authors of [16] to prove that Ramsey's theorem for strictly increasing numbers of colors forms a strictly increasing chain with respect to strong Weihrauch reducibility [16, Theorem 3.1]:

$$
\mathrm{RT}_{n, 2}<_{\mathrm{sW}} \mathrm{RT}_{n, 3}<_{\mathrm{sW}} \mathrm{RT}_{n, 3}<_{\mathrm{sW}} \ldots
$$

However, they left it an open question [16, Question 7.1] whether an analogous statement also holds for ordinary Weihrauch reducibility. We will be able to use our Theorem 3.24 on products to answer this question in the affirmative (see Theorem 4.22). Independently, similar results were obtained by Hirschfeldt and Jockusch [23] and Patey [32. Altogether, the diagram in Figure 1 displays how Ramsey's theorem for different cardinalities and colors is situated in the Weihrauch lattice.

We briefly describe the further organization of this article. In the following Section 2 we briefly present the basic concepts and definitions related to the Weihrauch lattice, and we collect a number of facts that are useful for our study. In Section 3 we introduce the uniform versions of Ramsey's theorem that we are going to consider in this study, and we establish a core lower bound in Theorem 3.5 that will be used to derive almost all other lower bound results. We also prove key results on products in Theorem 3.24 and on parallelization in Theorem 3.28 that also lead to our main lower bound results, which are formulated in Corollary 3.27 and Corollary 3.30. In Section 4 we discuss jumps of Ramsey's theorem together with upper bound results. In particular, we prove Theorem 4.3 , which is our main result on jumps and shows that the stable version of Ramsey's theorem can be seen as the jump of the colored version of the next smaller cardinality. These results lead to Corollary 4.14, which shows that composition with one jump of weak Konnig's lemma is sufficient to bring Ramsey's theorem from one cardinality to the next greater cardinality. From this result we can conclude our main upper bound result in Corollary 4.15, which shows that Ramsey's theorem of cardinality $n$ is reducible to the $n$-th jump of weak König's lemma. Together with our lower bound results this finally leads to the classification of the parallelization of Ramsey's theorem of cardinality $n$ as the $n$-th jump of weak König's lemma in Corollary 4.18 and to the classification of the exact Borel degree of Ramsey's theorem in Corollary 4.19. We also use these results to obtain the above mentioned result on increasing numbers of colors. In Section 5 we briefly discuss the special case of Ramsey's theorem for pairs, we summarize some known results, provide some new insights and formulate some open questions. In Section 6 we develop separation techniques for jumps, and we apply them to separate some uniform versions of Ramsey's theorem. Finally, in Section 7 we discuss the relation of closed and compact choice problems to Ramsey's theorem, which corresponds to the discussion of boundedness and induction principles in reverse mathematics.

\section{Preliminaries}

We use the theory of the Weihrauch lattice (as it has been developed in 3, 4, 5, 6, [7, 8]) as a framework for the uniform classification of the computational content of mathematical problems. We present a very brief introduction of the main concepts and refer the reader to [6] and [8] for a more detailed introduction.

Formally, the Weihrauch lattice is formed by equivalence classes of partial multivalued functions $f: \subseteq X \rightrightarrows Y$ on represented spaces $X, Y$, as defined below. We will simply call such functions problems here, and they are, in fact, computational challenges in the sense that for every $x \in \operatorname{dom}(f)$ the goal is to find some $y \in f(x)$. In this case $\operatorname{dom}(f)$ contains the admissible instances $x$ of the problem, and for each instance $x$ the set $f(x)$ contains the corresponding solutions.

For problems $f: \subseteq X \rightrightarrows Y, g: \subseteq Y \rightrightarrows Z$ we define the composition $g \circ f: \subseteq X \rightrightarrows Z$ by $g \circ f(x):=\{z \in Z:(\exists y \in f(x)) z \in g(y)\}$, where it is crucial to use the domain 
$\operatorname{dom}(g \circ f):=\{x \in X: f(x) \subseteq \operatorname{dom}(g)\}$. We also denote the composition briefly by $g f$.

A represented space $(X, \delta)$ is a set $X$ together with a representation, i.e., a surjective partial map $\delta: \subseteq \mathbb{N}^{\mathbb{N}} \rightarrow X$ that assigns names $p \in \mathbb{N}^{\mathbb{N}}$ to points $\delta(p)=x \in X$. Given a problem $f: \subseteq X \rightrightarrows Y$ on represented spaces $\left(X, \delta_{X}\right)$ and $\left(Y, \delta_{Y}\right)$ we say that a partial function $F: \subseteq \mathbb{N}^{\mathbb{N}} \rightarrow \mathbb{N}^{\mathbb{N}}$ realizes $f$, in symbols $F \vdash f$, if $\delta_{Y} F(p) \in f \delta_{X}(p)$ for all $p \in \operatorname{dom}\left(f \delta_{X}\right)$. Notions such as computability and continuity can now be transferred from Baire space to represented spaces via realizers. For instance, $f$ is called computable, if it admits a computable realizer $F$. We refer the reader to [9, 39. for further details.

The intuition behind Weihrauch reducibility is that $f \leq_{\mathrm{W}} g$ holds if there is a computational procedure for solving $f$ during which a single application of the computational resource $g$ is allowed. There are actually two slightly different formal versions of this reduction, which are both needed.

Definition 2.1 (Weihrauch reducibility). Let $f: \subseteq X \rightrightarrows Y$ and $g: \subseteq W \rightrightarrows Z$ be problems.

(1) $f$ is called Weihrauch reducible to $g$, in symbols $f \leq_{\mathrm{W}} g$, if there are computable $K: \subseteq X \rightrightarrows W, H: \subseteq X \times Z \rightrightarrows Y$ such that $\emptyset \neq H(x, g K(x)) \subseteq f(x)$ for all $x \in \operatorname{dom}(f)$.

(2) $f$ is called strongly Weihrauch reducible to $g$, in symbols $f \leq_{\mathrm{sW}} g$, if there are computable $K: \subseteq X \rightrightarrows W, H: \subseteq Z \rightrightarrows Y$ such that $\emptyset \neq H g K(x) \subseteq f(x)$ for all $x \in \operatorname{dom}(f)$.

In terms of realizers and functions on Baire space (strong) Weihrauch reducibility can also be rephrased as follows [20, Lemma 4.5]:

- $f \leq_{\mathrm{W}} g \Longleftrightarrow\left(\exists\right.$ computable $\left.H, K: \subseteq \mathbb{N}^{\mathbb{N}} \rightarrow \mathbb{N}^{\mathbb{N}}\right)(\forall G \vdash g) H\langle$ id, $G K\rangle \vdash f$.

- $f \leq_{\mathrm{sW}} g \Longleftrightarrow\left(\exists\right.$ computable $\left.H, K: \subseteq \mathbb{N}^{\mathbb{N}} \rightarrow \mathbb{N}^{\mathbb{N}}\right)(\forall G \vdash g) H G K \vdash f$.

Here id $: \mathbb{N}^{\mathbb{N}} \rightarrow \mathbb{N}^{\mathbb{N}}$ denotes the identity of Baire space. A relation between Weihrauch reducibility and strong Weihrauch reducibility can be established with the notion of a cylinder. A problem $f$ is called a cylinder if id $\times f \leq_{\mathrm{sW}} f$, which is equivalent to the property that $g \leq_{\mathrm{W}} f \Longleftrightarrow g \leq_{\mathrm{sW}} f$ holds for all problems $g$, 5 , Corollary 3.6].

Weihrauch reducibility induces a lattice with a rich and very natural algebraic structure. We briefly summarize some of these algebraic operations for problems $f: \subseteq X \rightrightarrows Y$ and $g: \subseteq W \rightrightarrows Z:$

- $f \times g: \subseteq X \times W \rightrightarrows Y \times Z$ is the product of $f$ and $g$ and represents the parallel evaluation of problem $f$ on some input $x$ and $g$ on some input $w$.

- $f \sqcup g: \subseteq X \sqcup W \rightrightarrows Y \sqcup Z$ is the coproduct of $f$ and $g$ and represents the alternative evaluation of $f$ on some input $x$ or $g$ on some input $w$ (where $X \sqcup W$ and $Y \sqcup Z$ denote the disjoint unions).

- $f * g:=\sup \left\{f_{0} \circ g_{0}: f_{0} \leq_{\mathrm{W}} f\right.$ and $\left.g_{0} \leq_{\mathrm{W}} g\right\}$ is the compositional product and represents the consecutive usage of the problem $f$ after the problem $g$.

- $f^{*}:=\bigsqcup_{n=0}^{\infty} f^{n}$ is the finite parallelization and allows an evaluation of the $n$-fold product $f^{n}$ for some arbitrary given $n \in \mathbb{N}$.

- $\widehat{f}: \subseteq X^{\mathbb{N}} \rightrightarrows Y^{\mathbb{N}}$ is the parallelization of $f$ and allows a parallel evaluation of countably many instances of $f$.

- $f^{\prime}: \subseteq X \rightrightarrows Y$ denotes the jump of $f$, which is formally the same problem as $f$, but the input representation $\delta_{X}$ of $X$ is replaced by its jump $\delta_{X}^{\prime}:=\delta_{X} \circ \lim$.

Here $\lim : \subseteq \mathbb{N}^{\mathbb{N}} \rightarrow \mathbb{N}^{\mathbb{N}},\left\langle p_{0}, p_{1}, p_{2}, \ldots\right\rangle \mapsto \lim _{i \rightarrow \infty} p_{i}$ is the limit map on Baire space for $p_{i} \in \mathbb{N}^{\mathbb{N}}$, where \langle\rangle denotes a standard infinite tupling function on Baire space. We recall that $\langle n, k\rangle:=\frac{1}{2}(n+k+1)(n+k)+k$, and inductively this can be extended to finite tuples by $\left\langle i_{n+1}, i_{n}, \ldots, i_{0}\right\rangle:=\left\langle i_{n+1},\left\langle i_{n}, \ldots, i_{0}\right\rangle\right\rangle$ for all $n \geq 1$ and $i_{0}, \ldots, i_{n+1} \in \mathbb{N}$. 
Likewise, we define $\left\langle p_{0}, p_{1}, p_{2}, \ldots\right\rangle \in \mathbb{N}^{\mathbb{N}}$ for $p_{i} \in \mathbb{N}^{\mathbb{N}}$ by $\left\langle p_{0}, p_{1}, p_{2}, \ldots\right\rangle\langle n, k\rangle:=p_{n}(k)$. Similarly as lim, we define the limit $\lim _{2^{\mathbb{N}}}: \subseteq 2^{\mathbb{N}} \rightarrow 2^{\mathbb{N}}$ on Cantor space $2^{\mathbb{N}}$. For finite sets $X \subseteq \mathbb{N}$ we denote by $\lim _{X}: \subseteq X^{\mathbb{N}} \rightarrow X$ the ordinary limit operation with respect to the discrete topology.

By $f^{(n)}$ we denote the $n$-fold jump of $f$, and we denote the $n$-fold compositional product of $f$ with itself by $f^{[n]}$, i.e., $f^{[0]}=\mathrm{id}, f^{[n+1]}:=f^{[n]} * f$. By $f^{n}: \subseteq X^{n} \rightrightarrows Y^{n}$ we denote the $n$-fold product $f \times \ldots \times f$ of $f$.

One can also define a sum operation $\sqcap$ that play the role of the infimum for ordinary Weihrauch reducibility $\leq_{\mathrm{w}}$. But we are not going to use this operation here. Altogether, $\leq_{\mathrm{W}}$ induces a lattice structure, and the resulting lattice is called Weihrauch lattice. This lattice is not complete as infinite suprema do not need to exist, but the special supremum $f * g$ is even a maximum that always exists [11, Proposition 31], and the compositional product $*$ is associative [11, Corollary 17]. Finite parallelization and parallelization are closure operators in the Weihrauch lattice. Further information on the algebraic structure can be found in [1].

For metric spaces $X$ we denote by $\boldsymbol{\Sigma}_{n}^{0}$ the corresponding class of Borel subsets of $X$ of level $n \geq 1$ [27]. We recall that a partial function $f: \subseteq X \rightarrow Y$ on metric spaces $X$ and $Y$ is called $\boldsymbol{\Sigma}_{n}^{0}-$ measurable, if for every open set $U \subseteq Y$ there exists a set $V \in \boldsymbol{\Sigma}_{n}^{0}$ such that $f^{-1}(U)=V \cap \operatorname{dom}(f)$, i.e., such that $f^{-1}(U)$ is a $\boldsymbol{\Sigma}_{n}^{0}$-set relative to the domain of $f$. In the case of $n=1$ we obtain exactly continuity. Likewise we define $f$ to be effectively $\boldsymbol{\Sigma}_{n}^{0}$-measurable if a corresponding $V$ can be uniformly computed from a given $U$ [2, Definition 3.5]. In this case we obtain exactly computability for $n=1$. The notion of (effective) $\boldsymbol{\Sigma}_{n}^{0}$-measurability can be transferred to problems $f: \subseteq X \rightrightarrows Y$ via realizers, i.e., $f$ is called (effectively) $\boldsymbol{\Sigma}_{n}^{0}-$ measurable, if it has a realizer $F: \subseteq \mathbb{N}^{\mathbb{N}} \rightarrow \mathbb{N}^{\mathbb{N}}$ that is (effectively) $\boldsymbol{\Sigma}_{n}^{0}$-measurable 2 It is easy to see that Weihrauch reducibility preserves (effective) $\boldsymbol{\Sigma}_{n}^{0}$-measurability downwards.

Fact 2.2. Let $f, g$ be problems and $n, k \geq 1$. Then

(1) $f$ (effectively) $\boldsymbol{\Sigma}_{n}^{0}-$ measurable and $g$ (effectively) $\boldsymbol{\Sigma}_{k}^{0}$-measurable $\Longrightarrow f * g$ (effectively) $\boldsymbol{\Sigma}_{n+k-1}^{0}-$ measurable.

(2) $f, g$ (effectively) $\boldsymbol{\Sigma}_{n}^{0}-$ measurable $\Longrightarrow f \times g$ (effectively) $\boldsymbol{\Sigma}_{n}^{0}-$ measurable.

(3) $f \leq_{\mathrm{W}} g$ and $g$ (effectively) $\boldsymbol{\Sigma}_{n}^{0}$-measurable $\Longrightarrow f$ (effectively) $\boldsymbol{\Sigma}_{n}^{0}$-measurable.

(4) $f \leq_{\mathrm{W}} \lim ^{[n]} \Longleftrightarrow f$ is effectively $\boldsymbol{\Sigma}_{n+1}^{0}{ }^{-}$measurable.

Proof. (1) and (2) follow form [2, Proposition 3.8]. (3) follows from (1) and (2) since $H\langle$ id, $G K\rangle$ is (effectively) $\boldsymbol{\Sigma}_{n}^{0}$-measurable, if $G: \subseteq \mathbb{N}^{\mathbb{N}} \rightarrow \mathbb{N}^{\mathbb{N}}$ is so and the functions

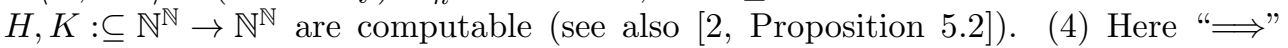
follows from (3) since lim is effectively $\boldsymbol{\Sigma}_{2}^{0}$-measurable [2, Proposition 9.1], and hence $\lim ^{[n]}$ is effectively $\boldsymbol{\Sigma}_{n+1}^{0}-$ measurable by (1). The inverse implication "£" follows with the help of the effective Banach-Hausdorff-Lebesgue theorem [2, Corollary 9.6] applied to the corresponding realizers on Baire space.

The last mentioned item can also be expressed such that $\lim ^{[n]}$ is effectively $\boldsymbol{\Sigma}_{n+1}^{0}{ }^{-}$ complete in the Weihrauch lattice.

An important problem in the Weihrauch lattice is closed choice $C_{X}: \subseteq \mathcal{A}_{-}(X) \rightrightarrows X$, $A \mapsto A$, which maps every closed set $A \subseteq X$ to its points. By $\mathcal{A}_{-}(X)$ we denote the set of closed subsets of a (computable) metric space $X$ with respect to negative information. This means that a closed set is essentially described by enumerating basic open balls that exhaust its complement. Intuitively, closed choice $C_{X}$ is the following problem: given a closed set $A$ by a description that lists everything that does not belong to $A$, find a point $x \in A$ (see 3 for further information and precise definitions).

\footnotetext{
${ }^{2}$ This definition yields a conservative extension of the notion of (effective) measurability to multivalued functions on represented space. It coincides for single-valued functions on computable Polish spaces with the usual notion of (effective) measurability as it is known from descriptive set theory [2].
} 
By BWT BW $_{X} \subseteq X^{\mathbb{N}} \rightrightarrows X$ we denote the Bolzano-Weierstraß theorem for the space $X$, which is the problem: given a sequence $\left(x_{n}\right)_{n}$ with a compact closure, find a cluster point of this sequence. This problem has been introduced and studied in detail in [7. If $X$ itself is compact, then this problem is total. We are particularly interested in finite versions of the Bolzano-Weierstraß theorem here. We recall that we identify $k \in \mathbb{N}$ with the set $X=\{0, \ldots, k-1\}$ in the following. By WKL we denote weak König's lemma, which is the problem WKL $: \subseteq \operatorname{Tr} \rightarrow 2^{\mathbb{N}}, T \mapsto[T]$ that maps every infinite binary tree $T$ to the set of its infinite paths. We summarize a number of facts that are of particular importance for us. Here and in the following we will occasionally use that jumps are monotone with respect to strong Weihrauch reducibility, i.e., $f \leq_{\mathrm{sW}} g \Longrightarrow f^{\prime} \leq_{\mathrm{sW}} g^{\prime}$ [7, Proposition 5.6(2)].

Fact 2.3. We obtain

(1) $\mathrm{BWT}_{k}^{(n-1)} \equiv{ }_{\mathrm{sW}} \mathrm{C}_{k}^{(n)}$ for all $k \in \mathbb{N}, n \geq 1$,

(2) $\mathrm{WKL}^{(n)} \equiv_{\mathrm{sW}} \widehat{\mathrm{C}_{k}^{(n)}}$ for all $k \geq 2$ and $n \in \mathbb{N}$,

(3) $\lim ^{(n)} \equiv_{\mathrm{sW}} \widehat{\lim _{k}^{(n)}}$ for all $k \geq 2, k=\mathbb{N}$ and $n \in \mathbb{N}$,

(4) $\lim _{k}^{(n)}<_{\mathrm{sW}} \mathrm{BWT}_{k}^{(n)}$ and $\lim _{k}^{(n)}<\mathrm{W} \mathrm{BWT}_{k}^{(n)}$ for all $k \geq 2, k=\mathbb{N}$ and $n \in \mathbb{N}$,

(5) $\mathrm{WKL}^{(n)}<_{\mathrm{sW}} \lim ^{(n)}<_{\mathrm{sW}} \mathrm{WKL}^{(n+1)}$ and $\mathrm{WKL}^{(n)}<_{\mathrm{W}} \lim ^{(n)}<_{\mathrm{W}} \mathrm{WKL}^{(n+1)}$ for all $n \in \mathbb{N}$,

(6) $\left(\mathrm{WKL}^{\prime}\right)^{[n]} \equiv_{\mathrm{W}} \mathrm{WKL}^{(n)}$ and $\lim ^{[n]} \equiv_{\mathrm{W}} \lim ^{(n-1)}$ for all $n \geq 1$.

Proof. (1) follows from [7, Theorem 9.4] (noting that the cluster point problem used there is identical to the Bolzano-Weierstraß theorem in the finite case). (2) follows since $\mathrm{WKL} \equiv_{\mathrm{sW}} \widehat{\mathrm{C}_{k}}$, which has essentially been proved in [5, Theorem 8.2], where LLPO is just a reformulation of $\mathrm{C}_{2}$ and from the fact that jumps and parallelizations commute by [7, Proposition 5.7(3)]. For (3) see [7, Fact 3.5]. (4) The reductions follow since the limit can be seen as a special case of the Bolzano-Weiertraß theorem [7, Proposition 11.21] and they are strict since $\lim _{k}^{(n)}$ is $\boldsymbol{\Sigma}_{n+2}^{0}-$ measurable, but $\mathrm{BWT}_{k}^{(n)}$ for $k \geq 2$ is not, see the proof of [6, Proposition 9.1]. The positive parts of the reductions in (5) are easy to see, and the strictness follows from (relativized versions) of the low basis theorem and (relativized versions) of the Kleene tree construction, respectively (see also [3, 7]). (6) The statement for WKL follows by induction from $\mathrm{WKL}^{(n+1)} \equiv_{\mathrm{W}} \mathrm{WKL}^{(n)} * \mathrm{WKL}^{\prime}$, where " $\leq_{\mathrm{W}}$ " holds since $\lim \leq_{\mathrm{W}} \mathrm{WKL}^{\prime}$ and the converse reduction follows from [7, Theorem 8.13]. The statement for lim follows for instance from [7, Corollary 5.16] since $\lim ^{(n)}$ is a cylinder.

\section{The Uniform Scenario, Lower Bounds and Products}

In this section we plan to introduce the uniform versions of Ramsey's theorem that we are going to study, and we will prove some basic facts about them. While in non-uniform settings such as reverse mathematics 37] certain information on infinite homogeneous sets can just be assumed to be available, we need to make this more explicit. For instance, it turned out to be useful to consider an enriched version $\mathrm{CRT}_{n, k}$ of Ramsey's theorem that provides additional information on the color of the produced infinite homogeneous set. In [12] the stable version of Ramsey's theorem $\mathrm{SRT}_{n, k}$ was introduced, which is a restriction of Ramsey's theorem that we consider too.

We need to introduce some notation first. For every $n \geq 1$ we assume that we use some total standard numbering $\vartheta_{n}: \mathbb{N} \rightarrow[\mathbb{N}]^{n}$ that can be defined, for instance, by $\vartheta_{n}\left\langle i_{0}, i_{1}, \ldots, i_{n-1}\right\rangle:=\left\{k+\sum_{j=0}^{k} i_{j}: k=0, \ldots, n-1\right\}$ for all $i_{0}, \ldots, i_{n-1} \in \mathbb{N}$, i.e., the set $\vartheta_{n}\left\langle i_{0}, i_{1}, \ldots, i_{n-1}\right\rangle$ contains the numbers $i_{0}<i_{0}+i_{1}+1<i_{0}+i_{1}+i_{2}+2<$ $\ldots<i_{0}+i_{1}+i_{n-1}+n-1$. However, we will not make any technical use of this 
specific definition of $\vartheta_{n}$. Occasionally, we use the notation $\left\{i_{0}<i_{1}<\ldots<i_{n-1}\right\}$ for a set $\left\{i_{0}, i_{1}, \ldots, i_{n-1}\right\} \in[\mathbb{N}]^{n}$ with the additional property that $i_{0}<i_{1}<\ldots<i_{n-1}$. By $\mathcal{C}_{n, k}$ we denote the set of colorings $c:[\mathbb{N}]^{n} \rightarrow k$ which is represented such that $p \in \mathbb{N}^{\mathbb{N}}$ is a name for $c$, if $p(i)=c\left(\vartheta_{n}(i)\right)$ (this is equivalent to using the natural function space representation $\left[\vartheta_{n} \rightarrow \mathrm{id}_{k}\right]$ as known in computable analysis [9, 39]). By $\mathcal{C}_{n, \mathbb{N}}$ we denote the set of all colorings $c:[\mathbb{N}]^{n} \rightarrow \mathbb{N}$ with a finite range, represented analogously. By $\mathcal{H}_{c}$ we denote the set of infinite homogeneous sets $M \subseteq \mathbb{N}$ for the coloring $c$. A coloring $c:[\mathbb{N}]^{n} \rightarrow k$ is called stable, if $\lim _{i \rightarrow \infty} c(A \cup\{i\})$ exists for all $A \in[\mathbb{N}]^{n-1}$. The expression $k \geq a, \mathbb{N}$ with $a \in \mathbb{N}$ is means $k \in\{x \in \mathbb{N}: x \geq a\} \cup\{\mathbb{N}\}$.

Definition 3.1 (Uniform variants of Ramsey's theorem). For all $n \geq 1$ and $k \geq 1, \mathbb{N}$ we define

(1) $\mathrm{RT}_{n, k}: \mathcal{C}_{n, k} \rightrightarrows 2^{\mathbb{N}}, \mathrm{RT}_{n, k}(c):=\mathcal{H}_{c}$,

(2) $\mathrm{CRT}_{n, k}: \mathcal{C}_{n, k} \rightrightarrows k \times 2^{\mathbb{N}}, \mathrm{CRT}_{n, k}(c):=\left\{(c(M), M): M \in \mathcal{H}_{c}\right\}$,

(3) $\mathrm{SRT}_{n, k}: \subseteq \mathcal{C}_{n, k} \rightrightarrows 2^{\mathbb{N}}, \mathrm{SRT}_{n, k}(c):=\mathrm{RT}_{n, k}(c)$, where $\operatorname{dom}\left(\mathrm{SRT}_{n, k}\right):=\left\{c \in \mathcal{C}_{n, k}: c\right.$ stable $\}$,

(4) $\mathrm{CSRT}_{n, k}: \subseteq \mathcal{C}_{n, k} \rightrightarrows k \times 2^{\mathbb{N}}, \mathrm{CSRT}_{n, k}(c):=\left\{(c(M), M): M \in \mathcal{H}_{c}\right\}$, where $\operatorname{dom}\left(\operatorname{CSRT}_{n, k}\right):=\left\{c \in \mathcal{C}_{n, k}: c\right.$ stable $\}$,

(5) $\mathrm{RT}_{n,+}:=\bigsqcup_{k \geq 1} \mathrm{RT}_{n, k}, \mathrm{RT}:=\bigsqcup_{n \geq 1} \mathrm{RT}_{n,+}$.

All formalized versions of Ramsey's theorem mentioned here are well-defined by Ramsey's theorem 1.1. We call $n$ the cardinality of the respective version of Ramsey's theorem. Here $\mathrm{CRT}_{n, k}$ enriches $\mathrm{RT}_{n, k}$ by the information on the color of the resulting infinite homogeneous set, and $\mathrm{SRT}_{n, k}$ is a restriction of $\mathrm{RT}_{n, k}$ defined only for stable colorings. The coproduct $\mathrm{RT}_{n,+}$ as well as $\mathrm{RT}_{n, \mathbb{N}}$ can both be seen as different uniform versions of the principle $(\forall k) \mathrm{RT}_{n, k}$ that is usually denoted by $\mathrm{RT}_{<\infty}^{n}$ in reverse mathematics (see, e.g., [22]). In the case of $\mathrm{RT}_{n, \mathbb{N}}$ the finite number of colors is left unspecified, whereas in the case of $\mathrm{RT}_{n,+}$, the number of colors is an input parameter.

We emphasize that we use Cantor space $2^{\mathbb{N}}$, i.e., the infinite homogeneous sets $M \in \mathcal{H}_{c}$ are represented via their characteristic functions $\chi_{M}: \mathbb{N} \rightarrow\{0,1\}$. However, by definition any infinite subset $A \subseteq M$ of an infinite homogeneous set $M \in \mathcal{H}_{c}$ is in $\mathcal{H}_{c}$ too, and given an enumeration of an infinite set $M$, we can find a characteristic function $\chi_{A}$ of an infinite subset $A \subseteq M$. This means that we can equivalently think about sets in $2^{\mathbb{N}}$ as being represented via enumerations 3

We obtain the following obvious strong reductions between the different versions of Ramsey's theorem.

Lemma $\quad 3.2 \quad$ (Basic reductions). $\quad \mathrm{SRT}_{n, k} \leq_{\mathrm{sW}} \mathrm{RT}_{n, k} \leq_{\mathrm{sW}} \mathrm{CRT}_{n, k}$ and $\mathrm{SRT}_{n, k} \leq_{\mathrm{sW}} \mathrm{CSRT}_{n, k} \leq_{\mathrm{sW}} \mathrm{CRT}_{n, k}$ for all $n \geq 1$ and $k \geq 1, \mathbb{N}$.

We note that the colored versions of Ramsey's theorem are Weihrauch equivalent to the corresponding uncolored versions. This is because given a coloring $c$ and an infinite homogeneous set $M \in \mathcal{H}_{c}$, we can easily compute $c(M)$ by choosing some points $i_{0}<i_{1}<\ldots<i_{n-1}$ in $M$ and by computing $c\left\{i_{0}, i_{1}, \ldots, i_{n-1}\right\}$. Hence, we obtain the following corollary.

Corollary 3.3. $\mathrm{RT}_{n, k} \equiv_{\mathrm{W}} \mathrm{CRT}_{n, k}$ and $\mathrm{SRT}_{n, k} \equiv_{\mathrm{W}} \mathrm{CSRT}_{n, k}$ for all $n \geq 1, k \geq 1, \mathbb{N}$.

The diagram in Figure2 illustrates the situation, and it displays further information on lower and upper bounds that is justified by proofs that we will only provide step by step. The diagram illustrates the non-trivial case $n \geq 2, k \geq 2, \mathbb{N}$ whereas the bottom cases where $n=1$ or $k=1$ are described in the following result.

\footnotetext{
${ }^{3}$ More formally, we could equivalently consider the output space of $\mathrm{RT}_{n, k}$ and its variants as $\mathcal{A}_{+}(\mathbb{N})$ or $\mathcal{A}(\mathbb{N})$, i.e., as space of subsets of $\mathbb{N}$ equipped with positive or full information, respectively, which corresponds topologically to the lower Fell topology and the Fell topology, respectively.
} 


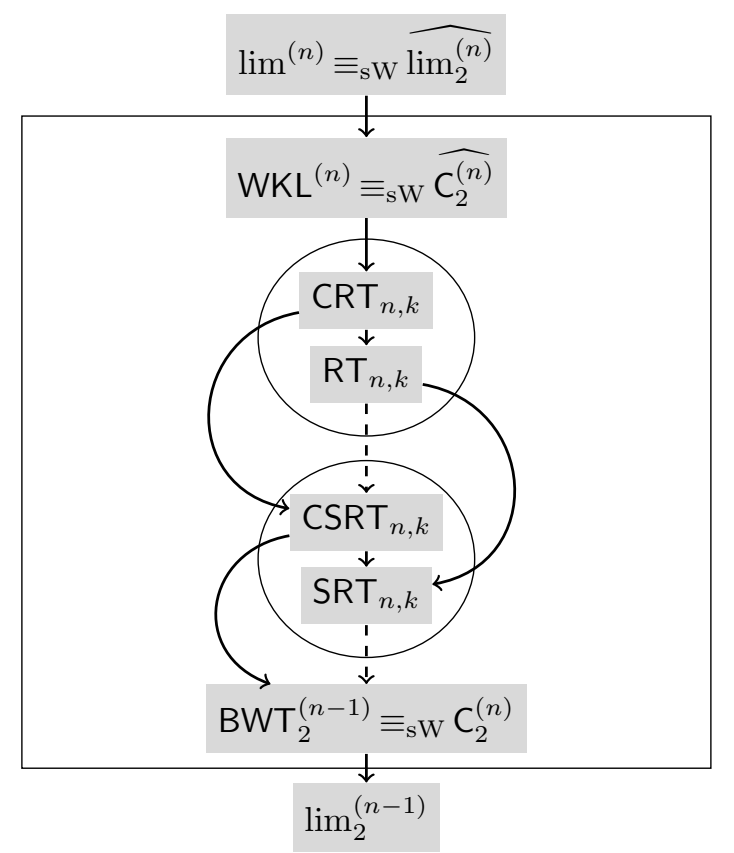

FiguRE 2. The degree of Ramsey's theorem for fixed $n \geq 2$ and $k \geq 2, \mathbb{N}$ in the Weihrauch lattice: all solid arrows indicate strong Weihrauch reductions against the direction of the arrow, all dashed arrows indicate ordinary Weihrauch reductions; the circle indicates what falls into a single Weihrauch degree, and the square box indicates what falls into a single parallelized Weihrauch degree.

Proposition 3.4 (The bottom cases). Let $n \geq 1$ and $k \geq 2, \mathbb{N}$. Then we obtain

(1) $\mathrm{CSRT}_{n, 1} \equiv_{\mathrm{sW}} \mathrm{SRT}_{n, 1} \equiv_{\mathrm{sW}} \mathrm{RT}_{n, 1} \equiv_{\mathrm{sW}} \mathrm{CRT}_{n, 1}$ are computable and not cylinders,

(2) $\lim _{k} \equiv_{\mathrm{W}} \mathrm{SRT}_{1, k}<\mathrm{W} \mathrm{BWT}_{k} \equiv_{\mathrm{W}} \mathrm{RT}_{1, k}$,

(3) $\lim _{k} \leq_{\mathrm{sW}} \mathrm{CSRT}_{1, k}$ and $\mathrm{BWT}_{k} \leq_{\mathrm{sW}} \mathrm{CRT}_{1, k}$.

Proof. We note that $\mathrm{CSRT}_{n, 1}, \mathrm{SRT}_{n, 1}, \mathrm{RT}_{n, 1}$ and $\mathrm{CRT}_{n, 1}$ can yield any infinite subset of $\mathbb{N}$ (together with the color 1 in the case of $\operatorname{CSRT}_{n, 1}, \mathrm{CRT}_{n, 1}$ ), and hence they are all constant as multi-valued functions, hence computable and strongly equivalent to each other. Since the identity cannot be strongly reduced to constant multi-valued problems, it follows that all the aforementioned problems are not cylinders. It is easy to see that $\lim _{k} \equiv_{\mathrm{W}} \mathrm{SRT}_{1, k}$ and $\mathrm{BWT}_{k} \equiv_{\mathrm{W}} \mathrm{RT}_{1, k}$, whereas $\lim _{k}<\mathrm{W} \mathrm{BWT}_{k}$ is given by Fact 2.3 (4). The strong reductions $\lim _{k} \leq_{\mathrm{sW}} \mathrm{CSRT}_{1, k}$ and $\mathrm{BWT}_{k} \leq_{\mathrm{sW}} \mathrm{CRT}_{1, k}$ are also clear.

This result demonstrates that the Ramsey theorem for cardinality 1 and $k$ colors $\mathrm{RT}_{1, k}$ is equivalent to the Bolzano-Weierstraß $\mathrm{BWT}_{k}$ for the space $k$, and hence the general Ramsey theorem can be seen as a generalization of the Bolzano-Weierstraß theorem for finite spaces. Next we want to prove the following interesting lower bound on the complexity of Ramsey's theorem.

Theorem 3.5 (Lower bound). $\mathrm{BWT}_{2}^{(n-1)} \equiv_{\mathrm{sW}} \mathrm{C}_{2}^{(n)} \leq_{\mathrm{sW}} \mathrm{CSRT}_{n, 2}$ for all $n \geq 2$. 
Proof. We note that by Fact $2.3 \mathrm{C}_{2}^{(n)} \equiv_{\mathrm{sW}} \mathrm{BWT}_{2}^{(n-1)} \equiv_{\mathrm{sW}} \mathrm{BWT}_{2} \circ \lim _{2^{\mathbb{N}}}^{[n-1]}$. Let $p \in$ $\operatorname{dom}\left(\mathrm{BWT}_{2} \circ \lim _{2^{\mathbb{N}}}^{[n-1]}\right)$ and $q:=\lim _{2^{\mathbb{N}}}^{[n-1]}(p)$. Then

$$
q\left(i_{0}\right)=\lim _{i_{1} \rightarrow \infty} \lim _{i_{2} \rightarrow \infty} \ldots \lim _{i_{n-1} \rightarrow \infty} p\left\langle i_{n-1}, \ldots, i_{0}\right\rangle
$$

for all $i_{0} \in \mathbb{N}$. We compute the coloring $c:[\mathbb{N}]^{n} \rightarrow 2$ with

$$
c\left\{i_{0}<i_{1}<\ldots<i_{n-1}\right\}:=p\left\langle i_{n-1}, i_{n-2}, \ldots, i_{1}, i_{0}\right\rangle .
$$

It is clear that $c$ is a stable coloring, and with the help of $\operatorname{CSRT}_{n, 2}$ we can compute $(c(M), M) \in \mathrm{CSRT}_{n, 2}(c)$. We claim that $c(M) \in \mathrm{BWT}_{2}(q)$. This proves $\mathrm{BWT}_{2}^{(n-1)} \leq_{\mathrm{sW}} \mathrm{CSRT}_{n, 2}$. We still need to prove the claim.

1. Case: $q$ is a convergent binary sequence, i.e., $x:=\lim _{2}(q) \in\{0,1\}$ exists.

In this case $q\left(i_{0}\right)=x$ for all sufficiently large $i_{0}$ and $\operatorname{BWT}_{2}(q)=\{x\}$. Since $M$ is infinite, there will be such a sufficiently large $i_{0} \in M$. Since $q=\lim _{2^{\mathbb{N}}}^{[n-1]}(p)$ it follows that there will be sufficiently large $i_{n-1}>\ldots>i_{1}>i_{0}$ in $M$ such that $p\left\langle i_{n-1}, i_{n-2}, \ldots, i_{1}, i_{0}\right\rangle=x$, and hence $c(M)=x \in\{x\}=\mathrm{BWT}_{2}(q)$.

2. Case: $q$ is not a convergent binary sequence.

In this case $\mathrm{BWT}_{2}(q)=\{0,1\}$ and $c(M) \in \mathrm{BWT}_{2}(q)$ hold automatically.

It is clear that Ramsey's theorem for any number of colors $k$ can be reduced to Ramsey's theorem for any greater number of colors. This is because any coloring $c:[\mathbb{N}]^{n} \rightarrow k$ can be seen as a coloring for any number $m \geq k$ of colors, and this idea applies to all versions of Ramsey's theorem that we have considered.

Lemma 3.6 (Increasing colors). For all $n, k \geq 1$ we obtain:

(1) $\mathrm{SRT}_{n, k} \leq_{\mathrm{sW}} \mathrm{SRT}_{n, k+1} \leq_{\mathrm{sW}} \mathrm{SRT}_{n, \mathbb{N}}$,

(2) $\mathrm{CSRT}_{n, k} \leq_{\mathrm{sW}} \mathrm{CSRT}_{n, k+1} \leq_{\mathrm{sW}} \mathrm{CSRT}_{n, \mathbb{N}}$,

(3) $\mathrm{RT}_{n, k} \leq_{\mathrm{sW}} \mathrm{RT}_{n, k+1} \leq_{\mathrm{sW}} \mathrm{RT}_{n,+} \leq_{\mathrm{sW}} \mathrm{RT}_{n, \mathbb{N}}$,

(4) $\mathrm{CRT}_{n, k} \leq_{\mathrm{sW}} \mathrm{CRT}_{n, k+1} \leq_{\mathrm{sW}} \mathrm{CRT}_{n, \mathbb{N}}$.

We will later come back to the question whether these reductions are strict. In particular, with Theorem 3.5 and Lemma 3.6 we have now established the lower bound that is indicated in the diagram in Figure 2

Corollary 3.7 (Lower bound). $\mathrm{BWT}_{2}^{(n-1)} \leq_{\mathrm{sW}} \mathrm{CSRT}_{n, k}$ for all $n \geq 2, k \geq 2, \mathbb{N}$ and $\lim _{2}^{(n-1)} \leq_{\mathrm{sW}} \mathrm{CSRT}_{n, k}$ for all $n \geq 1$ and $k \geq 2, \mathbb{N}$.

The second statement follows from the first one by Fact 2.3 in the case of $n \geq 2$ and follows from Proposition 3.4 in the case of $n=1$. We note that by Corollary 3.15below $\mathrm{CSRT}_{n, k}$ cannot be replaced by SRT ${ }_{n, k}$ in this result. It follows from Theorem 1.5 that the binary limit $\lim _{2}$ cannot be replaced by the limit on Baire space in the previous result. We recall that $\lim ^{(n-1)} \equiv_{\mathrm{sW}} \lim ^{[n]}$.

Corollary 3.8 (Limit avoidance). $\lim ^{(n-1)} \not \underline{\mathrm{W}}_{\mathrm{W}} \lim * \mathrm{RT}_{n, \mathbb{N}}$ for all $n \geq 2$.

Proof. Let us assume that $\lim ^{(n-1)} \leq_{\mathrm{W}} \lim * \mathrm{RT}_{n, \mathbb{N}}$ holds for some $n \geq 2$. Then $\lim ^{(n-1)}$ maps some computable $p \in \mathbb{N}^{\mathbb{N}}$ to $\emptyset^{(n)}$, and the reduction maps $p$ to a computable coloring $c:[\mathbb{N}]^{n} \rightarrow \mathbb{N}$ with finite range $(c)$. By Theorem 1.5 there exists an infinite homogeneous set $M \subseteq \mathbb{N}$ for $c$ such that $\emptyset^{(n)} \not_{\mathrm{T}} M^{\prime}$. Now the assumption is that there is a limit computation performed on $p$ and $M$ that produces $\emptyset^{(n)}$. But any result produced by such a limit computation can also be computed from $M^{\prime}$ since $p$ is computable (and for all computable functions $F, G: \subseteq \mathbb{N} \rightarrow \mathbb{N}$ there is a computable function $H: \subseteq \mathbb{N} \rightarrow \mathbb{N}$ such that $F \circ \lim \circ G=H \circ \mathrm{J})$. Hence, $\emptyset^{(n)} \leq_{\mathrm{T}} M^{\prime}$, which is a contradiction.

We obtain the following corollary since $\lim * \lim ^{(n-2)} \equiv_{\mathrm{W}} \lim ^{(n-1)}$, which in turn follows from [7, Corollary 5.17] since $*$ is associative. 
Corollary 3.9 (Limit avoidance). $\lim ^{(n-2)} \not_{\mathrm{W}} \mathrm{RT}_{n, \mathbb{N}}$ for all $n \geq 2$.

We recall that a problem $f$ is called parallelizable, if $f \equiv_{\mathrm{W}} \widehat{f}$. We can conclude that Ramsey's theorem is not parallelizable.

Corollary 3.10 (Parallelizability). $\mathrm{RT}_{n, k}$ and $\mathrm{SRT}_{n, k}$ are not parallelizable for all $n \geq 1$ and $k \geq 2, \mathbb{N}$.

Proof. By Corollaries 3.7 and 3.3 we have $\lim _{2}^{(n-1)} \leq_{\mathrm{W}} \mathrm{SRT}_{n, k}$ for $n \geq 1, k \geq 2, \mathbb{N}$. Let us assume that $\mathrm{SRT}_{n, k}$ is parallelizable. Since parallelization is a closure operator, this implies by Lemma 3.6

$$
\lim ^{(n-2)} \leq_{\mathrm{W}} \lim ^{(n-1)} \equiv_{\mathrm{W}} \widehat{\lim _{2}^{(n-1)}} \leq_{\mathrm{W}} \widehat{\mathrm{SRT}_{n, k}} \equiv_{\mathrm{W}} \mathrm{SRT}_{n, k} \leq_{\mathrm{W}} \mathrm{RT}_{n, \mathbb{N}},
$$

where the first equivalence holds by Fact 2.3 (3). This is a contradiction to Corollary 3.9. The proof for $\mathrm{RT}_{n, k}$ follows analogously.

We recall that a problem $f$ is called idempotent, if $f \equiv_{\mathrm{W}} f \times f$. The squashing theorem (Theorem 1.7) implies that every total, finitely tolerant and idempotent problem is parallelizable. Hence we can draw the following conclusion from Corollary 3.10 (this has also been stated in [16, Lemma 3.3]).

Corollary 3.11 (Idempotency). $\mathrm{RT}_{n, k}$ is not idempotent for all $n \geq 1$ and $k \geq 2$.

Another noticeable consequence of Corollary 3.7 is the following.

Corollary 3.12 (Cylinders). $\widehat{\mathrm{CSRT}_{n, k}^{(m)}}$ and $\widehat{\mathrm{CRT}_{n, k}^{(m)}}$ are cylinders for all $n \geq 1$, $k \geq 2, \mathbb{N}$ and $m \geq 0$.

The claim follows since id $\leq_{\mathrm{sW}} \lim \equiv_{\mathrm{sW}} \widehat{\lim _{2}} \leq_{\mathrm{sW}} \mathrm{CSRT}_{n, k}$ holds by Corollary 3.7 and Fact 2.3. The next lemma now formulates a simple finiteness condition that Ramsey's theorem satisfies and implies that Ramsey's theorem has very little uniform computational power.

Lemma 3.13 (Finite Intersection Lemma). Let $c_{i}:[\mathbb{N}]^{n} \rightarrow k$ be colorings for $i=$ $1, \ldots, m$ with $m, n \geq 1, k \geq 1, \mathbb{N}$. Then we obtain $\bigcap_{i=1}^{m} \mathrm{RT}_{n, k}\left(c_{i}\right) \neq \emptyset$.

Proof. We first consider $k \geq 1$. We use a bijection $\alpha:\{0,1, \ldots, k-1\}^{m} \rightarrow\left\{0,1, \ldots, k^{m}-\right.$ $1\}$ in order to construct a map $f:\left(\mathcal{C}_{n, k}\right)^{m} \rightarrow \mathcal{C}_{n, k^{m}}$ by

$$
f\left(c_{1}, \ldots, c_{m}\right)(A):=\alpha\left(c_{1}(A), \ldots, c_{m}(A)\right)
$$

for all colorings $c_{1}, \ldots, c_{m} \in \mathcal{C}_{n, k}$ and $A \in[\mathbb{N}]^{n}$. Given $c_{1}, \ldots, c_{m} \in \mathcal{C}_{n, k}$ we consider $c:=f\left(c_{1}, \ldots, c_{m}\right)$, and we claim that $\mathrm{RT}_{n, k^{m}}(c) \subseteq \bigcap_{i=1}^{m} \mathrm{RT}_{n, k}\left(c_{i}\right)$. To this end, let $M \in \mathrm{RT}_{n, k^{m}}(c)$ and $x:=c(M)$. If $\left(x_{1}, \ldots, x_{m}\right):=\alpha^{-1}(x)$, then we obtain $c_{i}(A)=x_{i}$ for all $i=1, \ldots, m$ and $A \in[M]^{n}$, and hence $M$ is homogeneous for all $c_{1}, \ldots, c_{m}$. This proves the claim. It follows by Ramsey's theorem 1.1 that $\mathrm{RT}_{n, k^{m}}(c) \neq \emptyset$.

We now consider the case $k=\mathbb{N}$. In this case we use Cantor's tupling function $\alpha: \mathbb{N}^{m} \rightarrow \mathbb{N}$ in order to construct a map $f:\left(\mathcal{C}_{n, \mathbb{N}}\right)^{m} \rightarrow \mathcal{C}_{n, \mathbb{N}}$ analogously as above. We obtain $\mathrm{RT}_{n, \mathbb{N}}(c) \subseteq \bigcap_{i=1}^{m} \mathrm{RT}_{n, \mathbb{N}}\left(c_{i}\right)$. It follows by Ramsey's theorem 1.1 that $\mathrm{RT}_{n, \mathbb{N}}(c) \neq \emptyset$.

In any case this proves the claim of the lemma.

This result could be generalized and proved in different possibly simpler ways. The specific construction used in our proof will be reused for the proof of Corollary 3.18,

We recall that for a multi-valued function $f: \subseteq X \rightrightarrows Y$ we have defined \# $f$, the cardinality of $f$, in [6] as the supremum of all cardinalities of sets $M \subseteq \operatorname{dom}(f)$ such that $\{f(x): x \in M\}$ is pairwise disjoint. The cardinality yields an invariant for strong Weihrauch reducibility, i.e., $f \leq_{\mathrm{sW}} g$ implies $\# f \leq \# g$ [6, Proposition 3.6]. Lemma 3.13 now implies the following perhaps surprising fact. 
Corollary 3.14 (Cardinality). $\# \mathrm{RT}_{n, k}^{(m)}=\# \widehat{\mathrm{RT}_{n, k}^{(m)}}=1$ for all $n \geq 1, k \geq 1, \mathbb{N}$ and $m \geq 0$.

In the case of the parallelization we only need to apply Lemma 3.13 pairwise to any component of the sequence. We recall that a multi-valued function $f$ was called discriminative in [8] if $\mathrm{C}_{2} \leq_{\mathrm{W}} f$ and indiscriminative otherwise. Likewise, we could call $f$ strongly discriminative if $\mathrm{C}_{2} \leq_{\mathrm{sW}} f$. We obtain $\# \mathrm{C}_{2}=2$ since $\{0\}$ and $\{1\}$ are in the domain of $\mathrm{C}_{2}$. Hence it follows that Corollary 3.14 implies in particular that Ramsey's theorem is not strongly discriminative, not even in its parallelized form.

Corollary 3.15 (Strong discrimination). $\mathrm{C}_{2} \underline{\mathrm{sW}}_{\mathrm{sW}} \widehat{\mathrm{RT}_{n, k}^{(m)}}$ for all $n \geq 1, k \geq 1, \mathbb{N}$ and $m \geq 0$.

Since $\mathrm{C}_{2}^{\prime} \equiv_{\mathrm{sW}} \mathrm{BWT}_{2}$ by Fact 2.3 , we can conclude that CSRT cannot be replaced by SRT in Theorem 3.5 and Corollary 3.7 (without simultaneously replacing the strong reduction by an ordinary one) We can also conclude from Corollary 3.14 that the parallelized uncolored versions of Ramsey's theorem are not cylinders since $\# \mathrm{id}=\left|\mathbb{N}^{\mathbb{N}}\right|$.

Corollary 3.16 (Cylinders). $\widehat{\mathrm{RT}_{n, k}^{(m)}}$ and $\widehat{\mathrm{SRT}_{n, k}^{(m)}}$ are not cylinders for all $n \geq 1$, $k \geq 1, \mathbb{N}$ and $m \geq 0$.

Since \#CSRT ${ }_{n, k} \geq k$ holds (because there are monochromatic colorings for each color), we can also conclude from Corollary 3.14 that the colored versions of Ramsey's theorem are not strongly equivalent to the uncolored ones (in the case of at least two colors).

Corollary 3.17. $\mathrm{CSRT}_{n, k} \not_{\mathrm{sW}} \widehat{\mathrm{RT}_{n, k}^{(m)}}$ for all $n \geq 1, k \geq 2, \mathbb{N}$ and $m \geq 0$.

The following result is a consequence of Lemma 3.13 and its proof. For a finite sequence $\left(f_{i}\right)_{i \leq m}$ of multi-valued functions $f_{i}: \subseteq X \rightrightarrows Y$ we denote the intersection by $\bigcap_{i=1}^{m} f_{i}: \subseteq X \rightrightarrows Y, x \mapsto \bigcap_{i=1}^{m} f_{i}(x)$, where $\operatorname{dom}\left(\bigcap_{i=1}^{m} f_{i}\right)$ contains all points $x \in X$ such that $\bigcap_{i=1}^{m} f_{i}(x) \neq \emptyset$. We recall that $f^{m}$ denotes the $m$-fold product of $f$ with respect to $\times$.

Corollary 3.18 (Products). For all $n, m, k \geq 1$ we obtain

(1) $\mathrm{RT}_{n, k}^{m} \leq_{\mathrm{sW}} \bigcap_{i=1}^{m} \mathrm{RT}_{n, k} \leq_{\mathrm{sW}} \mathrm{RT}_{n, k^{m}}$,

(2) $\mathrm{SRT}_{n, k}^{m} \leq_{\mathrm{sW}} \bigcap_{i=1}^{m} \mathrm{SRT}_{n, k} \leq_{\mathrm{sW}} \mathrm{SRT}_{n, k^{m}}$,

(3) $\mathrm{RT}_{n, \mathbb{N}}^{m} \leq_{\mathrm{sW}} \bigcap_{i=1}^{m} \mathrm{RT}_{n, \mathbb{N}} \leq_{\mathrm{sW}} \mathrm{RT}_{n, \mathbb{N}}$,

(4) $\mathrm{SRT}_{n, \mathbb{N}}^{m} \leq_{\mathrm{sW}} \bigcap_{i=1}^{m} \mathrm{SRT}_{n, \mathbb{N}} \leq_{\mathrm{sW}} \mathrm{SRT}_{n, \mathbb{N}}$.

Proof. The functions $f:\left(\mathcal{C}_{n, k}\right)^{m} \rightarrow \mathcal{C}_{n, k^{m}}$ and $f:\left(\mathcal{C}_{n, \mathbb{N}}\right)^{m} \rightarrow \mathcal{C}_{n, \mathbb{N}}$ constructed in the proof of Lemma 3.13 are computable, and hence they yield the reductions $\bigcap_{i=1}^{m} \mathrm{RT}_{n, k} \leq_{\mathrm{sW}} \mathrm{RT}_{n, k^{m}}$ and $\bigcap_{i=1}^{m} \mathrm{RT}_{n, \mathbb{N}} \leq_{\mathrm{sW}} \mathrm{RT}_{n, \mathbb{N}}$, respectively. Additionally, both maps $f$ have the property that they map stable colorings $c_{1}, \ldots, c_{m}$ to stable colorings $c:=f\left(c_{1}, \ldots, c_{m}\right)$, hence they also yield the corresponding reductions in the stable case. The other reductions follow from Lemma 3.13

We note that by [16, Proposition 2.1] we also have $\mathrm{RT}_{n, k} \times \mathrm{RT}_{n, l} \leq_{\mathrm{sW}} \mathrm{RT}_{n, k l}$ for all $n, k, l \geq 1$. We also note that the following result is implicitly included in [16. Section 1] (the proof given there is for the case $l=1$ and $k=2$ and can be generalized straightforwardly).

\footnotetext{
${ }^{4}$ It follows from Corollary 5.23 below that in Corollary $3.15 \mathrm{C}_{2}$ cannot be replaced by $\mathrm{ACC}_{\mathbb{N}}$, as defined in 8 .
} 
Proposition 3.19 (Compositional products). $\mathrm{RT}_{n, k+l} \leq_{\mathrm{W}} \mathrm{RT}_{n, k} * \mathrm{RT}_{n, l+1}$ for all $n, k, l \geq 1$.

From Corollary 3.18 we can directly conclude that Ramsey's theorem for an unspecified finite number of colors is idempotent.

Corollary 3.20 (Idempotency). $\mathrm{RT}_{n, \mathbb{N}}$ and $\mathrm{SRT}_{n, \mathbb{N}}$ are idempotent for all $n \geq 1$.

Since the sequence $\left(f_{m}\right)_{m}$ of maps $f_{m}:\left(\mathcal{C}_{n, \mathbb{N}}\right)^{m} \rightarrow \mathcal{C}_{n, \mathbb{N}}$ from the proof of Corollary 3.18 is uniformly computable, we obtain the following corollary.

Corollary 3.21 (Finite parallelization). $\mathrm{RT}_{n, k}^{*} \leq{ }_{\mathrm{W}} \mathrm{RT}_{n,+}$ and $\mathrm{SRT}_{n, k}^{*} \leq{ }_{\mathrm{W}} \mathrm{SRT}_{n,+}$ for all $n \geq 1$ and $k \geq 1, \mathbb{N}$.

We note that $\mathrm{RT}_{n, k}^{*}=\bigsqcup_{m>0} \mathrm{RT}_{n, k}^{m}$, where $\mathrm{RT}_{n, k}^{0}=\mathrm{id}$, and hence we obtain only an ordinary Weihrauch reduction in the previous result. Corollary 3.21 leads to the obvious question, whether additional factors can make up for color increases.

Question 3.22 (Colors and factors). Does $\mathrm{RT}_{n, k}^{*} \equiv_{\mathrm{W}} \mathrm{RT}_{n,+}$ hold for $n, k \geq 2$ ?

We note that the equivalence is known to hold in the case of $n=1$.

Proposition 3.23 (Colors and factors). $\mathrm{RT}_{1, n+1} \leq_{\mathrm{W}} \mathrm{RT}_{1,2}^{n}$ for all $n \geq 1$ and, in particular, $\mathrm{RT}_{1,2}^{*} \equiv_{\mathrm{W}} \mathrm{RT}_{1,+}$.

Proof. In [33, Theorem 32] it was proved that $\mathrm{C}_{n+1} \leq_{\mathrm{sW}} \mathrm{C}_{2}^{n}$ holds for all $n \geq 1$ (only " $\leq_{\mathrm{W}}$ " was claimed but the proof shows " $\leq_{\mathrm{sW}}$ "). By Fact 2.3 (1) this implies $\mathrm{BWT}_{n+1} \leq_{\mathrm{sW}} \mathrm{BWT}_{2}^{n}$, since jumps are monotone with respect to $\leq_{\mathrm{sW}}$. Hence, with Proposition 3.4 we obtain $\mathrm{RT}_{1, n+1} \equiv_{\mathrm{W}} \mathrm{BWT}_{n+1} \leq_{\mathrm{W}} \mathrm{BWT}_{2}^{n} \equiv_{\mathrm{W}} \mathrm{RT}_{1,2}^{n}$. Since this reduction is uniform in $n$, we can conclude that

$$
\mathrm{RT}_{1,+}=\bigsqcup_{k \geq 1} \mathrm{RT}_{1, k} \leq \mathrm{W} \bigsqcup_{k \geq 1} \mathrm{RT}_{1,2}^{k-1} \equiv_{\mathrm{W}} \mathrm{RT}_{1,2}^{*}
$$

The other direction follows since the reductions in Corollary 3.18 are uniform and hence

$$
\mathrm{RT}_{1,2}^{*}=\bigsqcup_{m \in \mathbb{N}} \mathrm{RT}_{1,2}^{m} \leq \mathrm{W} \bigsqcup_{m \in \mathbb{N}} \mathrm{RT}_{1,2^{m}} \leq \mathrm{W} \bigsqcup_{k \geq 1} \mathrm{RT}_{1, k}=\mathrm{RT}_{1,+} \cdot
$$

As a consequence of the next result we obtain that any unspecified finite number of colors can be reduced to two colors for the price of an increase of the cardinality. Simultaneously, the following theorem also gives us a handle to show that the complexity of Ramsey's theorem increases with increasing numbers of colors.

Theorem $3.24 \quad$ (Products). $\mathrm{RT}_{n, \mathbb{N}} \times \mathrm{RT}_{n+1, k} \leq_{\mathrm{sW}} \mathrm{RT}_{n+1, k+1}$ and $\mathrm{SRT}_{n, \mathbb{N}} \times \mathrm{SRT}_{n+1, k} \leq_{\mathrm{sW}} \mathrm{SRT}_{n+1, k+1}$ for all $n \geq 1$ and $k \geq 1, \mathbb{N}$.

Proof. We start with the first reduction. Given a coloring $c_{1}:[\mathbb{N}]^{n} \rightarrow \mathbb{N}$ with finite range and a coloring $c_{2}:[\mathbb{N}]^{n+1} \rightarrow k$ we construct a coloring $c^{+}:[\mathbb{N}]^{n+1} \rightarrow k+1$ as follows:

$$
c^{+}(A):= \begin{cases}c_{2}(A) & \text { if } A \text { is homogeneous for } c_{1} \\ k & \text { otherwise }\end{cases}
$$

for all $A \in[\mathbb{N}]^{n+1}$. Let $M \in \mathrm{RT}_{n+1, k+1}\left(c^{+}\right)$and let $p: \mathbb{N} \rightarrow \mathbb{N}$ be the principal function of $M$. Then we define a coloring $c_{p}:[\mathbb{N}]^{n} \rightarrow \mathbb{N}$ by $c_{p}(A):=c_{1}(p(A))$ for all $A \in[\mathbb{N}]^{n}$. By construction, $c_{p}$ has finite range too. Let $B \in \mathrm{RT}_{n, \mathbb{N}}\left(c_{p}\right)$. Then $p(B)$ is homogeneous for $c_{1}$ and $p(B) \subseteq M$. Hence any $A \in[p(B)]^{n+1}$ is also homogeneous for $c_{1}$, which implies $c^{+}(A)=c_{2}(A)$ and hence $c^{+}(M)=c_{2}(A)<k$. This implies $M \in \mathrm{RT}_{n+1, k}\left(c_{2}\right)$ and all $A \in[M]^{n+1}$ are homogeneous for $c_{1}$. We claim that this implies $M \in \mathrm{RT}_{n, \mathbb{N}}\left(c_{1}\right)$. The claim yields $\mathrm{RT}_{n+1, k+1}\left(c^{+}\right) \subseteq \mathrm{RT}_{n, \mathbb{N}}\left(c_{1}\right) \cap \mathrm{RT}_{n+1, k}\left(c_{2}\right)$, and hence the desired reduction follows. 
We still need to prove the claim. To this end we show that all $A \in[M]^{n+1}$ are homogeneous for $c_{1}$ with respect to one fixed color. Firstly, we note that for every two $A, B \in[M]^{n+1}$ there is a finite sequence $A_{1}, \ldots, A_{l} \in[M]^{n+1}$ such that $A_{1}=A$, $A_{l}=B$ and $\left|A_{i} \cap A_{i+1}\right| \geq n$ for all $i=1, \ldots, l-1$. This is because each element of $A$ can be replaced step by step by one element of $B$. Now we fix some $i \in\{1, \ldots, l-1\}$. Since $A_{i}$ and $A_{i+1}$ are homogeneous for $c_{1}$ by assumption and they share an $n$-element subset, it is clear that $c_{1}\left(A_{i}\right)=c_{1}\left(A_{i+1}\right)$. Since this holds for all $i \in\{1, \ldots, l-1\}$, we obtain $c_{1}(A)=c_{1}(B)$. This means that all $A \in[M]^{n+1}$ are homogeneous for $c_{1}$ with respect to the same fixed color, and hence, in particular, all $A \in[M]^{n}$ share the same color with respect to $c_{1}$, i.e., $M \in \mathrm{RT}_{n, \mathbb{N}}\left(c_{1}\right)$.

Now we still show that the same construction also proves the second reduction regarding stable colorings. For this it suffices to show that $c^{+}:[\mathbb{N}]^{n+1} \rightarrow k+1$ is stable for all stable colorings $c_{1}:[\mathbb{N}]^{n} \rightarrow \mathbb{N}$ and $c_{2}:[\mathbb{N}]^{n+1} \rightarrow k$. For this purpose let $c_{1}, c_{2}$ be stable, and let $A \in[\mathbb{N}]^{n}$. Then $[A]^{n-1}=\left\{B_{0}, B_{1}, \ldots, B_{n-1}\right\}$ and for each $i=0, \ldots, n-1$ there is some $l_{i} \geq \max \left(B_{i}\right)$ and some $x_{i} \in \mathbb{N}$ such that $c_{1}\left(B_{i} \cup\{j\}\right)=x_{i}$ for $j>l_{i}$, since $c_{1}$ is stable. There is also some $l_{n} \geq \max (A)$ and some $x_{n} \in k$ such that $c_{2}(A \cup\{j\})=x_{n}$ for $j>l_{n}$, since $c_{2}$ is stable. Let $l:=\max \left\{l_{0}, \ldots, l_{n-1}, l_{n}\right\}$. Then

$$
c^{+}(A \cup\{j\})= \begin{cases}c_{2}(A \cup\{j\}) & \text { if } c_{1}(A)=x_{0}=x_{1}=\ldots=x_{n-1} \\ k & \text { otherwise }\end{cases}
$$

for all $j>l$. Hence $c^{+}$is stable.

We note that in the case of $k=1$ we get the following corollary.

Corollary 3.25 (Color reduction). $\mathrm{RT}_{n, \mathbb{N}} \leq_{\mathrm{sW}} \mathrm{RT}_{n+1,2}$ and $\mathrm{SRT}_{n, \mathbb{N}} \leq_{\mathrm{sW}} \mathrm{SRT}_{n+1,2}$ for all $n \geq 1$.

We note that the increase of the cardinality from $n$ to $n+1$ in this corollary is necessary in the case of RT by Corollaries 3.11 and 3.21

We mention that the proof of Theorem 3.24 also shows that the coloring $c^{+}$constructed therein has only infinite homogeneous sets of colors other than $k$. In the case of $k=1$ this means that only infinite homogeneous sets of color 0 occur. We denote by $0-\mathrm{SRT}_{n+1,2}$ the stable version $\mathrm{SRT}_{n+1,2}$ of Ramsey's theorem restricted to colorings that only admit infinite homogeneous sets of color 0 . We obtain the following corollary, which we consider only as a technical step towards the proof of Corollary 4.5 in the next section (and hence we do not phrase it for RT in place of SRT).

Corollary 3.26 (Color reduction). $\mathrm{SRT}_{n, \mathbb{N}} \leq_{\mathrm{sW}} 0-\mathrm{SRT}_{n+1,2}$ for all $n \geq 1$.

With the help of Proposition 3.4(2) in the case $k=\mathbb{N}$ we obtain the following corollary of Corollary 3.25 .

Corollary 3.27 (Discrete lower bounds). $\lim _{\mathbb{N}} \leq_{\mathrm{W}} \mathrm{SRT}_{2,2}$ and $\mathrm{BWT}_{\mathbb{N}} \leq_{\mathrm{W}} \mathrm{RT}_{2,2}$.

Since Ramsey's theorem is not parallelizable by Corollary 3.10, it is clear that some increase in the cardinality is necessary in order to accommodate the parallelization. We will show that it is sufficient and necessary to increase the cardinality by 2 . The next result shows that this is sufficient, and it uses the ideas that have already been applied in the proofs of Corollary 3.18 and Theorem 3.24 .

Theorem $\quad \mathbf{3 . 2 8} \quad$ (Delayed Parallelization). $\widehat{\mathrm{RT}_{n, k}} \leq_{\mathrm{sW}} \mathrm{RT}_{n+2,2}$ and $\widehat{\mathrm{SRT}_{n, k}} \leq{ }_{\mathrm{sW}} \mathrm{SRT}_{n+2,2}$ for all $n \geq 1$ and $k \geq 1, \mathbb{N}$.

Proof. We start with the reduction $\widehat{\mathrm{RT}_{n, k}} \leq_{\mathrm{sW}} \mathrm{RT}_{n+2,2}$ for $k \geq 1$. Given a sequence $\left(c_{i}\right)_{i}$ of colorings $c_{i}:[\mathbb{N}]^{n} \rightarrow k$, we want to determine infinite homogeneous sets $M_{i}$ for all of them in parallel, using $\mathrm{RT}_{n+2,2}$. The sequence $\left(f_{m}\right)_{m}$ of functions $f_{m}:\left(\mathcal{C}_{n, k}\right)^{m} \rightarrow \mathcal{C}_{n, k^{m}}$, defined as in the proof of Lemma 3.13 , is computable, and we 
use it to compute a sequence $\left(d_{m}\right)_{m}$ of colorings $d_{m} \in \mathcal{C}_{n, k^{m}}$ by $d_{m}:=f_{m}\left(c_{0}, \ldots, c_{m-1}\right)$. Given the sequence $\left(d_{m}\right)_{m}$, we can compute a sequence $\left(d_{m}^{+}\right)_{m}$ of colorings $d_{m}^{+}$: $[\mathbb{N}]^{n+1} \rightarrow 2$ by

$$
d_{m}^{+}(A):= \begin{cases}0 & \text { if } A \text { is homogeneous for } d_{m} \\ 1 & \text { otherwise }\end{cases}
$$

for all $A \in[\mathbb{N}]^{n+1}$. Now, in a final step we compute a coloring $c:[\mathbb{N}]^{n+2} \rightarrow 2$ with

$$
c(\{m\} \cup A):=d_{m}^{+}(A)
$$

for all $A \in[\mathbb{N}]^{n+1}$ and $m<\min (A)$. Given an infinite homogeneous set $M \in$ $\mathrm{RT}_{n+2,2}(c)$ we determine a sequence $\left(M_{i}\right)_{i}$ as follows: for each fixed $i \in \mathbb{N}$ we first search for a number $m>i$ in $M$, and then we let $M_{i}:=\{x \in M: x>m\}$. It follows from the definition of $c$ that $M_{i}$ is homogeneous for $d_{m}^{+}$, and following the reasoning in the proof of Theorem 3.24, we obtain that $M_{i}$ is also homogeneous for $d_{m}$. Following the reasoning in the proof of Lemma 3.13, we finally conclude that $M_{i} \in \bigcap_{j=0}^{m-1} \mathrm{RT}_{n, k}\left(c_{j}\right)$, hence, in particular, $M_{i} \in \mathrm{RT}_{n, k}\left(c_{i}\right)$, which was to be proved.

We note that the entire construction preserves stability. As shown in the proof of Corollary 3.18, the function $f$ preserves stability. Hence, given a sequence $\left(c_{i}\right)_{i}$ of stable colorings, also the sequence $\left(d_{m}\right)_{m}$ consists of stable colorings. Likewise, it was shown in the proof of Theorem 3.24 that in this case also the sequence $\left(d_{m}^{+}\right)_{m}$ consists of stable colorings. It follows immediately from the construction of $c$ that also $c$ is stable, since

$$
\lim _{j \rightarrow \infty} c(\{m\} \cup A \cup\{j\})=\lim _{j \rightarrow \infty} d_{m}(A \cup\{j\})
$$

for all $A \in[\mathbb{N}]^{n}$ and $m<\min (A)$. Altogether, this proves $\widehat{\mathrm{SRT}_{n, k}} \leq_{\mathrm{sW}} \mathrm{SRT}_{n+2,2}$. The case $k=\mathbb{N}$ can be handled analogously.

Again the observation made after Corollary 3.25] applies: the colorings $d_{m}^{+}$can only have infinite homogeneous sets of color 0 , and hence also $c$ can only have infinite homogeneous sets of color 0 . This yields the following corollary, which we consider as a technical step towards the proof of Corollary 4.6 in the next section (hence we do not phrase it for RT in place of SRT).

Corollary 3.29 (Delayed Parallelization). $\widehat{\mathrm{SRT}_{n, k}} \leq_{\mathrm{sW}} 0-\mathrm{SRT}_{n+2,2}$ for all $n \geq 1$ and $k \geq 1, \mathbb{N}$.

Theorem 3.28 in combination with some other results also yields the following lower bounds of versions of Ramsey's theorem.

Corollary $\quad 3.30 \quad$ (Lower bounds). $\quad \lim \leq_{\mathrm{W}} \mathrm{SRT}_{3,2}, \quad \mathrm{WKL}^{\prime} \leq_{\mathrm{W}} \mathrm{RT}_{3,2} \quad$ and $\mathrm{WKL}^{(n)} \leq_{\mathrm{W}} \mathrm{SRT}_{n+2,2}$ for all $n \geq 2$.

Proof. With the help of Fact 2.3, Corollary 3.3 and Theorem 3.28 we obtain:

- $\lim \equiv_{\mathrm{W}} \widehat{\lim _{2}} \equiv_{\mathrm{W}} \widehat{\mathrm{SRT}_{1,2}} \leq{ }_{\mathrm{W}} \mathrm{SRT}_{3,2}$,

- $\mathrm{WKL}^{\prime} \equiv_{\mathrm{W}} \widehat{\mathrm{C}_{2}^{\prime}} \leq_{\mathrm{W}} \widehat{\mathrm{RT}_{1,2}} \leq_{\mathrm{W}} \mathrm{RT}_{3,2}$,

- $\mathrm{WKL}^{(n)} \equiv_{\mathrm{W}} \widehat{\mathrm{C}_{2}^{(n)}} \leq{ }_{\mathrm{W}} \widehat{\mathrm{SRT}_{n, 2}} \leq{ }_{\mathrm{W}} \mathrm{SRT}_{n+2,2}$ for $n \geq 2$.

For the first statement we have additionally used Proposition 3.4 and in the latter two cases Theorem 3.5

Corollary [3.30] generalizes [23. Corollary 2.3] (see Corollary [5.14). Corollary [3.9 together with Corollary 3.30 show that both corollaries are optimal in the sense that $\lim ^{(n-2)}$ cannot be replaced by $\lim ^{(n-3)}$ in the statement of the Corollary 3.9. and $\mathrm{WKL}^{(n)}$ cannot be replaced by $\lim ^{(n)}$ in the statement of Corollary 3.30. In particular, $n+2$ in Theorem 3.28 is also optimal and cannot be replaced by $n+1$. However, the following question remains open. 
Question 3.31. Does $\mathrm{WKL}^{\prime} \leq_{\mathrm{W}} \mathrm{SRT}_{3,2}$ hold?

As a combination of Corollaries 3.27 and 3.30 we obtain the following result on versions of Ramsey's theorem above the limit and the Bolzano-Weierstraß theorem for $\{0,1\}, \mathbb{N}$ and $\mathbb{N}^{\mathbb{N}}$, respectively.

Corollary 3.32 (Cones). $\lim _{2} \leq_{\mathrm{sW}} \mathrm{BWT}_{2} \leq_{\mathrm{W}} \mathrm{RT}_{1,2}, \lim _{\mathbb{N}} \leq_{\mathrm{sW}} \mathrm{BWT}_{\mathbb{N}} \leq_{\mathrm{W}} \mathrm{RT}_{2,2}$ and $\lim \leq_{\mathrm{sW}} \mathrm{BWT}_{\mathbb{N}^{\mathbb{N}}} \leq_{\mathrm{W}} \mathrm{RT}_{3,2}$.

Proof. We note that $\lim _{X} \leq_{\mathrm{W}}$ BWT $_{X}$ holds for arbitrary computable metric spaces $X$ by [7, Proposition 11.21]. Hence the first reduction chain follows with Proposition 3.4. the second one follows from Corollary 3.27 and the third one from Corollary 3.30 with the extra observation that $\mathrm{WKL}^{\prime} \equiv_{\mathrm{W}} \mathrm{BWT}_{\mathbb{N}^{\mathbb{N}}}$ by [7, Corollaries 11.6 and 11.7].

\section{Jumps, Increasing Cardinality and Color and Upper Bounds}

The purpose of this section is to provide a useful upper bound on Ramsey's theorem. Simultaneously, we will demonstrate how the complexity of Ramsey's theorem increases with increasing cardinality. The proof is subdivided into several steps. The first and crucial step made by Theorem 4.3 is interesting by itself and connects the jump of the colored version of Ramsey's theorem with the stable version of the next greater cardinality. This is one result where the usage of the colored version of Ramsey's theorem is essential. We start with a result that prepares the first direction of this theorem.

Proposition 4.1 (Jumps). $\mathrm{CRT}_{n, k}^{\prime} \leq_{\mathrm{sW}} \mathrm{CSRT}_{n+1, k}$ for all $n \geq 1$ and $k \geq 1, \mathbb{N}$.

Proof. Let $\left(c_{i}\right)_{i}$ be a sequence that converges to a coloring $c_{\infty}:[\mathbb{N}]^{n} \rightarrow k$. Without loss of generality we can assume that the $c_{i}$ are colorings $c_{i}:[\mathbb{N}]^{n} \rightarrow k$ themselves (this can easily be achieved by replacing every value $\geq k$ in the range of $c_{i}$ by 0 ). We compute the coloring $c:[\mathbb{N}]^{n+1} \rightarrow k$ with

$$
c(A \cup\{i\}):=c_{i}(A)
$$

for all $A \in[\mathbb{N}]^{n}$ and $i>\max (A)$. Then $c$ is stable, and we claim that $\mathrm{RT}_{n+1, k}(c) \subseteq$ $\mathrm{RT}_{n, k}\left(c_{\infty}\right)$. To this end, let $M \in \mathrm{RT}_{n+1, k}(c)$, and let $A \in[M]^{n}$. Since $M$ is infinite and $\lim _{i \rightarrow \infty} c(A \cup\{i\})=c_{\infty}(A)$, we obtain $c(M)=c_{\infty}(A)$. Since this holds for all $A \in$ $[M]^{n}$, we obtain that $M$ is homogeneous for $c_{\infty}$, i.e., $M \in \mathrm{RT}_{n, k}\left(c_{\infty}\right)$. We note that we also obtain $c_{\infty}(M)=c(M)$, and hence this proves $\mathrm{CRT}_{n, k}^{\prime} \leq_{\mathrm{sW}} \mathrm{CSRT}_{n+1, k}$.

We obtain the following corollary, since the jump operator is monotone with respect to strong reductions by [7, Proposition 5.6].

Corollary 4.2 (Jumps). $\mathrm{RT}_{n, k}^{(m)} \leq_{\mathrm{W}} \mathrm{SRT}_{n+m, k}$ for all $m, n \geq 1, k \geq 1, \mathbb{N}$.

It is not immediately clear whether this result also holds with a strong reduction. Now we are prepared to formulate our main result on jumps of Ramsey's theorem.

Theorem 4.3 (Jumps). $\mathrm{CRT}_{n, k}^{\prime} \equiv_{\mathrm{W}} \mathrm{SRT}_{n+1, k}$ for all $n \geq 1, k \geq 1, \mathbb{N}$.

Proof. By Proposition 4.1 and Corollary 3.3 we obtain $\mathrm{CRT}_{n, k}^{\prime} \leq_{\mathrm{W}} \mathrm{SRT}_{n+1, k}$, and it remains to prove $\mathrm{SRT}_{n+1, k} \leq_{\mathrm{W}} \mathrm{CRT}_{n, k}^{\prime}$. Let $c:[\mathbb{N}]^{n+1} \rightarrow k$ be a stable coloring. Then we can define a sequence $\left(c_{i}\right)_{i}$ of colorings $c_{i}:[\mathbb{N}]^{n} \rightarrow k$ by

$$
c_{i}(A):= \begin{cases}c(A \cup\{i\}) & \text { if } i>\max (A) \\ 0 & \text { otherwise }\end{cases}
$$

for all $A \in[\mathbb{N}]^{n}$. Then $\left(c_{i}\right)_{i}$ is a converging sequence of colorings, and let the limit be denoted by $c_{\infty}:[\mathbb{N}]^{n} \rightarrow k$. With the help of $\mathrm{CRT}_{n, k}$ we can compute $\left(c_{\infty}\left(M_{\infty}\right), M_{\infty}\right) \in \mathrm{CRT}_{n, k}\left(c_{\infty}\right)$. We will now describe how we can use this set $M_{\infty}$ together with $c_{\infty}$ and $c$ in order to computably enumerate an infinite homogeneous 
set $M \in \mathrm{SRT}_{n+1, k}(c)$. The set $M=\bigcup_{i=0}^{\infty} M_{i}$ will be defined inductively using sets $M_{i} \in\left[M_{\infty}\right]^{n+i}$. We start with choosing some $M_{0} \in\left[M_{\infty}\right]^{n}$. Then we continue in steps $i=0,1,2 \ldots$ as follows:

Let us assume that we have $M_{i} \in\left[M_{\infty}\right]^{n+i}$. For all $A \in\left[M_{i}\right]^{n}$ we obtain

$$
\lim _{j \rightarrow \infty} c(A \cup\{j\})=c_{\infty}(A)=c_{\infty}\left(M_{\infty}\right) .
$$

Hence, we can effectively find an $m>\max \left(M_{i}\right)$ such that $M_{i+1}:=M_{i} \cup\{m\}$ satisfies $c\left(M_{i+1}\right)=c_{\infty}\left(M_{\infty}\right)$.

Since all the sets $M_{i}$ with $i \geq 1$ are homogeneous sets for $c$ with the same color $c_{\infty}\left(M_{\infty}\right)$, the set $M:=\bigcup_{i=0}^{\infty} M_{i}$ is an infinite homogeneous set for $c$ with $c(M)=$ $c_{\infty}\left(M_{\infty}\right)$. This proves the desired reduction $\mathrm{SRT}_{n+1, k} \leq \mathrm{W} \mathrm{CRT}_{n, k}^{\prime}$.

It follows from Corollaries 3.12 and 3.16 that the equivalence in Theorem 4.3 cannot be replaced by a strong equivalence. We note that the color provided by $\mathrm{CRT}_{n, k}^{\prime}$ is not needed if the color of the infinite homogeneous set that is to be constructed is known in advance. Hence, the proof of Theorem 4.3 yields also the following result.

Corollary 4.4 (Jumps in the case of known color). $0-\mathrm{SRT}_{n+1,2} \leq{ }_{\mathrm{W}} \mathrm{RT}_{n, 2}^{\prime}$ for all $n \geq 1$.

We note that Corollaries 4.4 and 3.26 allow us to improve the bound given in Corollary 3.25 somewhat.

Corollary 4.5 (Color reduction with jumps). $\mathrm{SRT}_{n, \mathbb{N}} \leq_{\mathrm{sW}} \mathrm{RT}_{n, 2}^{\prime}$ for all $n \geq 1$.

We collect all a number of lower bound results in the following corollary that strengthen some earlier results mentioned in Corollaries 3.27 and 3.30 .

Corollary 4.6 (Lower bounds with jumps). $\lim _{\mathbb{N}} \leq_{W} \mathrm{RT}_{1,2}^{\prime}, \quad \lim \leq_{\mathrm{W}} \mathrm{RT}_{2,2}^{\prime}$ and $\mathrm{WKL}^{(n)} \leq_{\mathrm{W}} \mathrm{RT}_{n+1,2}^{\prime}$ for all $n \geq 2$.

Proof. We obtain $\lim _{\mathbb{N}} \equiv_{\mathrm{W}} \mathrm{SRT}_{1, \mathbb{N}} \leq_{\mathrm{W}} \mathrm{RT}_{1,2}^{\prime}$ by Proposition 3.4 and Corollary 4.5 , With the help of Fact 2.3. Proposition 3.4. Theorem 3.5 and Corollaries 3.29 and 4.4 we obtain

- $\lim \equiv_{\mathrm{W}} \widehat{\lim _{2}} \equiv_{\mathrm{W}} \widehat{\mathrm{SRT}_{1,2}} \leq_{\mathrm{sW}} 0-\mathrm{SRT}_{3,2} \leq_{\mathrm{W}} \mathrm{RT}_{2,2}^{\prime}$,

- $\mathrm{WKL}^{(n)} \equiv_{\mathrm{W}} \widehat{\mathrm{C}_{2}^{(n)}} \leq{ }_{\mathrm{W}} \widehat{\mathrm{SRT}_{n, 2}} \leq_{\mathrm{W}} 0-\mathrm{SRT}_{n+2,2} \leq_{\mathrm{W}} \mathrm{RT}_{n+1,2}^{\prime}$ for $n \geq 2$.

Analogously to Question 3.31 the following question remains open.

Question 4.7. Does $\mathrm{WKL}^{\prime} \leq_{\mathrm{W}} \mathrm{RT}_{2,2}^{\prime}$ hold?

Roughly speaking, Theorem 4.3 indicates that any increase in the cardinality of Ramsey's theorem corresponds to a jump. We can also conclude from Corollary 4.2 that Ramsey's theorem is increasing with respect to increasing cardinality.

Lemma 4.8 (Increasing cardinality). $\mathrm{SRT}_{n, k} \leq_{\mathrm{W}} \mathrm{RT}_{n, k} \leq_{\mathrm{W}} \mathrm{SRT}_{n+1, k} \leq_{\mathrm{W}} \mathrm{RT}_{n+1, k}$ for all $n \geq 1, k \geq 1, \mathbb{N}$.

Proof. It follows from Lemma 3.2 and Corollaries 4.2 and 3.3 that $\mathrm{SRT}_{n, k} \leq \mathrm{W}_{\mathrm{W}} \mathrm{RT}_{n, k} \leq_{\mathrm{W}} \mathrm{RT}_{n, k}^{\prime} \leq_{\mathrm{sW}} \mathrm{CRT}_{n, k}^{\prime} \leq_{\mathrm{W}} \mathrm{SRT}_{n+1, k} \leq_{\mathrm{W}} \mathrm{RT}_{n+1, k}$.

We will soon see in Corollary 4.21 and 5.3 that the reductions in this lemma are all strict in certain cases. We note that $\mathrm{CRT}_{n, k}^{\prime} \leq{ }_{\mathrm{W}} \mathrm{CRT}_{n, k} * \lim \equiv_{\mathrm{W}} \mathrm{RT}_{n, k} * \lim$. The latter problem is very stable and has several useful descriptions.

Lemma 4.9 (Jump of the cylindrification). For all $n \geq 1, k \geq 1, \mathbb{N}$ we obtain $\mathrm{RT}_{n, k} * \lim \equiv_{\mathrm{W}}\left(\mathrm{RT}_{n, k} \times \mathrm{id}\right)^{\prime} \equiv_{\mathrm{W}} \mathrm{RT}_{n, k}^{\prime} \times \lim$. 
Proof. The second equivalence holds since jumps and products commute by [7, Proposition $5.7(2)$ ]. Since $\mathrm{RT}_{n, k} \times$ id is a cylinder we have by [7, Corollary 5.17]

$\left(\mathrm{RT}_{n, k} \times \mathrm{id}\right)^{\prime} \equiv_{\mathrm{W}}\left(\mathrm{RT}_{n, k} \times \mathrm{id}\right) * \lim \equiv_{\mathrm{W}}\left(\mathrm{RT}_{n, k} * \lim \right) \times \lim \equiv_{\mathrm{W}} \mathrm{RT}_{n, k} * \lim$.

The latter two equivalences hold since lim is idempotent.

Since $\mathrm{RT}_{n, k} \times$ id is the cylindrification of Ramsey's theorem, this lemma characterizes the jump of the cylindrification of Ramsey's theorem up to Weihrauch equivalence. In particular, we obtain the following consequence of Theorem 4.3 .

Corollary 4.10. $\mathrm{SRT}_{n+1, k} \leq_{\mathrm{W}} \mathrm{RT}_{n, k} * \lim$ for all $n \geq 1, k \geq 1, \mathbb{N}$.

This result is the crucial step towards our upper bound result. The next step involves a usage of the cohesiveness problem. We recall that a set $A \subseteq \mathbb{N}$ is called cohesive for a sequence $\left(R_{i}\right)_{i}$ of sets $R_{i} \subseteq \mathbb{N}$ if $A \cap R_{i}$ or $A \cap\left(\mathbb{N} \backslash R_{i}\right)$ is finite for each $i \in \mathbb{N}$. In other words, up to finitely many exceptions, $A$ is fully included in any $R_{i}$ or its complement. By $\mathrm{COH}:\left(2^{\mathbb{N}}\right)^{\mathbb{N}} \rightrightarrows 2^{\mathbb{N}}$ we denote the cohesiveness problem, where $\operatorname{COH}\left(R_{i}\right)$ contains all sets $A \subseteq \mathbb{N}$ that are cohesive for $\left(R_{i}\right)_{i}$. The uniform computational content of $\mathrm{COH}$ has already been studied in [16, 8, 17. The relevance of the cohesiveness problem for Ramsey's theorem has originally been noticed by Cholak et al. who proved over $\mathrm{RCA}_{0}$ that $\mathrm{RT}_{2,2} \Longleftrightarrow \mathrm{SRT}_{2,2} \wedge \mathrm{COH}$ [12, Lemma 7.11] (see [13] for a correction). We use the same idea to prove the following, which was in the case of $n=k=2$ also observed by Dorais et al. [16].

Proposition 4.11. $\mathrm{RT}_{n, k} \leq_{\mathrm{W}} \mathrm{SRT}_{n, k} * \mathrm{COH}$ for all $n \geq 1, k \geq 1, \mathbb{N}$.

Proof. We fix some $n, k \geq 1$. Given a coloring $c:[\mathbb{N}]^{n} \rightarrow k$ we compute a sequence $\left(R_{i}\right)_{i}$ of sets $R_{i} \subseteq \mathbb{N}$ as follows:

$$
R_{\langle i, j\rangle}:=\left\{r \geq \max \vartheta_{n-1}(i): c\left(\vartheta_{n-1}(i) \cup\{r\}\right)=j\right\}
$$

for all $i, j \in \mathbb{N}$. Here $\vartheta_{n-1}$ denotes the numbering of $[\mathbb{N}]^{n-1}$ introduced in Section 3 , With the help of $\mathrm{COH}$ we can compute an infinite cohesive set $Y \in \mathrm{COH}\left(R_{i}\right)_{i}$ for the sequence $\left(R_{i}\right)_{i}$. Let $\sigma: \mathbb{N} \rightarrow \mathbb{N}$ be the principal function of $Y$. Since $Y$ is cohesive for $\left(R_{i}\right)_{i}$ and range $(c)$ is finite, it follows that for all $B \in[\mathbb{N}]^{n-1}$ there is some $j \in \mathbb{N}$ such that $c(B \cup\{r\})=j$ holds for almost all $r \in Y$. Hence, the coloring $c_{\sigma}:[\mathbb{N}]^{n} \rightarrow k$, defined by

$$
c_{\sigma}(A):=c(\sigma(A))
$$

for all $A \in[\mathbb{N}]^{n}$ is stable. With the help of $\mathrm{SRT}_{n, k}$ we can compute an infinite homogeneous set $M_{\sigma} \in \mathrm{SRT}_{n, k}\left(c_{\sigma}\right)$. It is clear that $M:=\sigma\left(M_{\sigma}\right)$ is an infinite homogeneous set for $c$.

Now we can combine Corollary 4.10 with Proposition 4.11 in both possible orders in order to obtain the following result. We recall that the compositional product $*$ is associative [11, Proposition 31].

Corollary 4.12. For all $n \geq 1, k \geq 1, \mathbb{N}$ we obtain

(1) $\mathrm{RT}_{n+1, k} \leq \mathrm{W} \mathrm{RT}_{n, k} * \lim * \mathrm{COH}$,

(2) $\mathrm{SRT}_{n+1, k} \leq \mathrm{W} \mathrm{SRT}_{n, k} * \mathrm{COH} * \lim$.

The diagram in Figure 3 illustrates the situation. The first bound given by Corollary 4.12 is particularly useful, since the following result was proved in 8, Corollary 14.14].

Fact 4.13. $\mathrm{WKL}^{\prime} \equiv_{\mathrm{W}} \lim * \mathrm{COH}$.

Fact 4.13 together with Corollary 4.12 yields the following.

Corollary 4.14 (Induction). $\mathrm{RT}_{n+1, k} \leq_{\mathrm{W}} \mathrm{RT}_{n, k} * \mathrm{WKL}^{\prime}$ for all $n \geq 1, k \geq 1, \mathbb{N}$. 


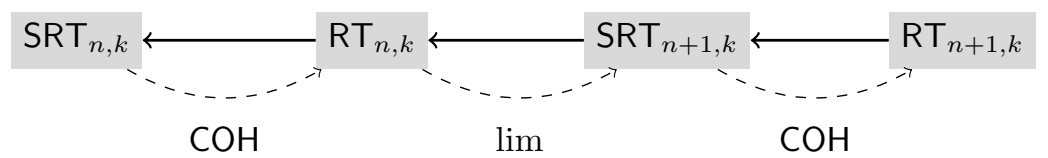

FiguRE 3. Upper bounds for Ramsey's theorem for $n \geq 2, k \geq 2, \mathbb{N}$ : all solid arrows indicated strong Weihrauch reductions against the direction of the arrow, all circled dashed arrows are labeled with an upper bound of the inverse implication.

This corollary can be interpreted such that $\mathrm{WKL}^{\prime}$ is sufficient to transfer $\mathrm{RT}_{n, k}$ into $\mathrm{RT}_{n+1, k}$. An obvious question is whether $\mathrm{WKL}^{\prime}$ is minimal with this property. It follows from Proposition 5.1 below and Lemma 4.9 that $\mathrm{WKL}^{\prime}$ cannot be replaced by lim in Corollary 4.14

Corollary 4.14 could also be proved directly using so-called Erdős-Rado trees 5 It reflects the fact that Ramsey's theorem can be proved inductively, where the induction base follows from the Bolzano-Weierstraß theorem for the $k$-point space by $\mathrm{RT}_{1, k} \equiv_{\mathrm{W}} \mathrm{BWT}_{k} \leq_{\mathrm{W}} \mathrm{WKL}^{\prime}$, which holds by Proposition 3.4 and Fact 2.3. Altogether, this means that we can derive the following result from Corollary 4.14 and Fact 2.3 (6).

Corollary 4.15 (Upper bound). $\mathrm{RT}_{n, k} \leq_{\mathrm{sW}} \mathrm{WKL}^{(n)}$ for all $n \geq 1, k \geq 1, \mathbb{N}$.

We note that we get a strong reduction here, since $\mathrm{WKL}^{(n)}$ is a cylinder. The upper bound is tight in terms of the number of jumps and also up to parallelization. Using the upper bound from Corollary 4.15 together with the lower bound from Corollary 3.30 yields the following characterization of Ramsey's theorem RT.

Corollary 4.16. $\mathrm{RT} \equiv_{\mathrm{W}} \bigsqcup_{n=0}^{\infty} \lim ^{(n)} \equiv_{\mathrm{W}} \bigsqcup_{n=0}^{\infty} \mathrm{WKL}^{(n)}$.

This degree corresponds to the class $\mathrm{ACA}_{0}^{\prime}$ in reverse mathematics (see [22, Theorem 6.27]). We call a problem $f$ countably irreducible if $f \leq_{\mathrm{W}} \bigsqcup_{n=0}^{\infty} g_{n}$ implies $f \leq_{\mathrm{W}} g_{n}$ for some $n \in \mathbb{N} 6$ Since it is clear that $C_{\mathbb{N}^{N}}$ is countably irreducible [3, Corollary 5.6], and it is easy to see that $\bigsqcup_{n=0}^{\infty} \lim ^{(n)} \leq_{\mathrm{W}} \mathrm{C}_{\mathbb{N}^{N}}$, we obtain the following corollary.

Corollary 4.17. $\mathrm{RT}<_{\mathrm{W}} \mathrm{C}_{\mathbb{N}^{\mathrm{N}}}$.

Here $C_{\mathbb{N}^{N}}$ can be seen as a possible counterpart of $A T R_{0}$ in reverse mathematics, in the sense that some statements that are equivalent to $A T R_{0}$ over $R C A_{0}$ in reverse mathematics turn out to be equivalent to $C_{\mathbb{N}^{\mathbb{N}}}$ in the Weihrauch lattice if interpreted as problems 7 We obtain the following characterization of the parallelization of Ramsey's theorem.

Corollary 4.18 (Parallelization). $\widehat{\mathrm{RT}_{n, k}} \equiv_{\mathrm{W}} \mathrm{WKL}^{(n)} \equiv_{\mathrm{sW}} \widehat{\mathrm{CRT}_{n, k}}$ for all $n \geq 1, k \geq$ $2, \mathbb{N}$ and $\widehat{\mathrm{SRT}_{n, k}} \equiv_{\mathrm{W}} \mathrm{WKL}^{(n)} \equiv_{\mathrm{sW}} \widehat{\mathrm{CRRT}}_{n, k}$ for all $n \geq 2, k \geq 2, \mathbb{N}$.

Proof. Using Fact 2.3. Corollary 3.3, the lower bound from Corollary 3.7 the upper bound from Corollary 4.15 and the fact that parallelization is a closure operator we obtain the following reduction chain for $n \geq 2, k \geq 2, \mathbb{N}$ :

$$
\mathrm{WKL}^{(n)} \equiv_{\mathrm{sW}} \widehat{\mathrm{C}_{2}^{(n)}} \equiv_{\mathrm{sW}} \widehat{\mathrm{BWT}_{2}^{(n-1)}} \leq_{\mathrm{sW}} \widehat{\mathrm{CSRT}_{n, k}} \leq_{\mathrm{W}} \widehat{\mathrm{SRT}_{n, k}} \leq \leq_{\mathrm{sW}} \widehat{\mathrm{RT}_{n, k}} \leq_{\mathrm{sW}} \mathrm{WKL}^{(n)}
$$

\footnotetext{
${ }^{5}$ This approach has been used in 34 Proposition 6.6.1]; for a proof theoretic analysis of Ramsey's theorem this method has been applied in [28, 29, 1, and in reverse mathematics it has been used to prove that Ramsey's theorem is provable over $\mathrm{ACA}_{0}$; see [19] for the Erdős-Rado method in general.

${ }^{6}$ This property has been called "join-irreducible" in previous publications, but formally it is a strengthening of join-irreducibility in the lattice theoretic sense. It is also not identical to "countable join-irreducibility" since the countable coproduct is not a supremum in general.

${ }^{7}$ Results in this direction are not yet published, but this emerged during a discussion at a recent Dagstuhl seminar on the subject.
} 
which implies $\widehat{\mathrm{SRT}_{n, k}} \equiv_{\mathrm{W}} \widehat{\mathrm{RT}_{n, k}} \equiv_{\mathrm{W}} \mathrm{WKL}^{(n)}$, and the corresponding strong equivalences $\widehat{C S R T}_{n, k} \equiv_{\mathrm{sW}} \widehat{\mathrm{CRT}_{n, k}} \equiv_{\mathrm{sW}} \mathrm{WKL}^{(n)}$ follow, since the colored versions of Ramsey's theorem are cylinders by Corollary 3.12 , and $\mathrm{WKL}^{(n)}$ is a cylinder too. The proof for $n=1$ follows similarly using Proposition 3.4.

We mention that $\widehat{\mathrm{SRT}_{1, k}} \equiv_{\mathrm{W}} \lim \equiv_{\mathrm{sW}} \mathrm{CSRT}_{1, k}$ holds for $k \geq 2, \mathbb{N}$ by Corollary 3.7 . By Corollary 3.16 the parallelized uncolored versions of Ramsey's theorem are not cylinders, and hence the Weihrauch equivalences $\equiv_{\mathrm{W}}$ in the previous corollary cannot be replaced by strong ones $\equiv_{\mathrm{sW}}$.

Since $\mathrm{WKL}^{(n)} \leq_{\mathrm{W}} \lim ^{(n)}$ and $\mathrm{WKL}^{(n)} \mathbb{L}_{\mathrm{W}} \lim ^{(n-1)}$ for all $n \geq 1$ by Fact 2.3 and $\lim ^{(n)}$ is effectively $\boldsymbol{\Sigma}_{n+2}^{0}$-complete by Facts 2.3 and 2.2 . we obtain the following corollary that characterizes the Borel complexity of Ramsey's theorem.

Corollary 4.19 (Borel complexity). $\mathrm{SRT}_{n, k}$ and $\mathrm{RT}_{n, k}$ are both effectively $\boldsymbol{\Sigma}_{n+2}^{0}$ measurable, but not effectively $\boldsymbol{\Sigma}_{n+1}^{0}$-measurable for all $n \geq 2, k \geq 2, \mathbb{N}$.

Both positive statements and the negative statement on $\mathrm{RT}_{n, k}$ also hold for $n=1$. By an application of Facts 2.2 and 2.3 we can also rephrase the negative statements as follows.

Corollary 4.20. $\mathrm{SRT}_{n, k} \mathbb{W}_{\mathrm{W}} \lim ^{(n-1)}$ for $n \geq 2$ and $\mathrm{RT}_{n, k} \mathbb{L}_{\mathrm{W}} \lim ^{(n-1)}$ for $n \geq 1$ and both for $k \geq 2, \mathbb{N}$.

Since effective $\boldsymbol{\Sigma}_{n}^{0}$-measurability is preserved downwards by Weihrauch reducibility by Fact 2.2. this in turn implies that Ramsey's theorem actually forms a strictly increasing chain with increasing cardinality.

Corollary 4.21 (Increasing cardinality). $\mathrm{RT}_{n, k}<_{\mathrm{W}} \mathrm{RT}_{n+1,2}, \mathrm{RT}_{n, k}<_{\mathrm{sW}} \mathrm{RT}_{n+1,2}$, $\mathrm{SRT}_{n, k}<\mathrm{W} \mathrm{SRT}_{n+1,2}$ and $\mathrm{SRT}_{n, k}<_{\mathrm{sW}} \mathrm{SRT}_{n+1,2}$ for all $n \geq 1$ and $k \geq 2, \mathbb{N}$.

Here the positive parts of the reduction hold by Corollary 3.25. The separations (at least in the case of RT) do also follow from classical non-uniform separation results of Jockusch [26] who showed that every computable instance of $\mathrm{RT}_{n, k}$ has a $\emptyset^{(n)_{-}}$ computable solution, whereas there are computable instance of $\mathrm{RT}_{n+1, k}$ that have no $\emptyset^{(n)}$-computable solution. We can also draw some conclusions on increasing numbers of colors. In [16, Theorem 3.1] Dorais et al. have proved that $\mathrm{RT}_{n, k}<_{\mathrm{sW}} \mathrm{RT}_{n, k+1}$ holds for all $n, k \geq 1$. Their main tool was a version of the squashing theorem (Theorem 1.7) for strong Weihrauch reducibility. With the help of Theorem 3.24 we can strengthen this result to ordinary Weihrauch reducibility, which answers [16, Question 7.1]. This result was independently obtained by Hirschfeldt and Jockusch [23, Theorem 3.3] and Patey [32, Corollary 3.15].

Theorem 4.22 (Increasing numbers of colors). $\mathrm{RT}_{n, k}<_{\mathrm{W}} \mathrm{RT}_{n, k+1}$ for all $n, k \geq 1$.

Proof. Let us assume that $\mathrm{RT}_{n, 2} \times \mathrm{RT}_{n+1, k} \leq \mathrm{W} \mathrm{RT}_{n+1, k}$ holds for some $n, k \geq 1$. Then by the squashing theorem (Theorem 1.7) we obtain $\overline{\mathrm{RT}_{n, 2}} \leq \mathrm{W} \mathrm{RT}_{n+1, k}$, and hence by Corollary 4.18

$$
\lim ^{(n-1)} \leq_{\mathrm{W}} \mathrm{WKL}^{(n)} \equiv_{\mathrm{W}} \widehat{\mathrm{RT}_{n, 2}} \leq{ }_{\mathrm{W}} \mathrm{RT}_{n+1, k}
$$

in contradiction to Corollary 3.9. Hence $\mathrm{RT}_{n, 2} \times \mathrm{RT}_{n+1, k} \not \mathrm{W}_{\mathrm{W}} \mathrm{RT}_{n+1, k}$ for all $n, k \geq 1$. On the other hand, we have $\mathrm{RT}_{n, 2} \times \mathrm{RT}_{n+1, k} \leq_{\mathrm{W}} \mathrm{RT}_{n+1, k+1}$ by Theorem 3.24. This implies $\mathrm{RT}_{n+1, k}<\mathrm{W} \mathrm{RT}_{n+1, k+1}$ for all $n, k \geq 1$. The claim $\mathrm{RT}_{1, k}<\mathrm{W} \mathrm{RT}_{1, k+1}$ for all $k \geq 1$ was already known [7, Theorem 13.4] via Proposition 3.4.

From this result we can also conclude that the two uniform versions of $\mathrm{RT}_{<\infty}^{n}$ are not equivalent. We recall that a problem $f: \subseteq X \rightrightarrows Y$ is called a fractal, if there is an $F: \subseteq \mathbb{N}^{\mathbb{N}} \rightrightarrows \mathbb{N}^{\mathbb{N}}$ with $F \equiv_{\mathrm{W}} f$ and $\left.F\right|_{A} \equiv_{\mathrm{W}} f$ for all clopen $A \subseteq \mathbb{N}^{\mathbb{N}}$ such that 
$A \cap \operatorname{dom}(F) \neq \emptyset$. Analogously, strong fractals are defined with $\equiv_{\mathrm{sW}}$ in place of $\equiv_{\mathrm{W}}$. It is easy to see that $\mathrm{RT}_{n, \mathbb{N}}$ and $\mathrm{SRT}_{n, \mathbb{N}}$ are fractals and hence countably irreducible by [7, Proposition 2.6], and thus the strictness of the reductions given in the following corollary follows from Theorem 4.22 .

Corollary 4.23 (Arbitrary numbers of colors). $\mathrm{RT}_{n,+}<_{\mathrm{W}} \mathrm{RT}_{n, \mathbb{N}}$ and $\mathrm{SRT}_{n,+}<\mathrm{W} \mathrm{SRT}_{n, \mathbb{N}}$ for all $n \geq 1$.

Another consequence of the squashing theorem (Theorem 1.7) is the following result that shows that the complexity of Ramsey's theorem also grows with an increasing number of factors. This generalizes Corollary 3.11.

Proposition 4.24 (Increasing number of factors). $\mathrm{RT}_{n, k}^{m}<\mathrm{W} \mathrm{RT}_{n, k}^{m+1}$ for all $n, m \geq 1$ and $k \geq 2$.

Proof. Let us assume that $\mathrm{RT}_{n, k}^{m+1} \equiv_{\mathrm{W}} \mathrm{RT}_{n, k} \times \mathrm{RT}_{n, k}^{m} \leq \mathrm{W} \mathrm{RT}_{n, k}^{m}$ holds for some $n, m \geq$ 1 and $k \geq 2$. Then by the squashing theorem (Theorem[1.7) we obtain $\widehat{\mathrm{RT}_{n, k}} \leq \mathrm{W} \mathrm{RT}_{n, k}^{m}$, and hence by Corollaries $3.21,3.25$ and 4.18 we can conclude

$$
\lim ^{(n-1)} \leq_{\mathrm{W}} \mathrm{WKL}^{(n)} \equiv_{\mathrm{W}} \widehat{\mathrm{RT}_{n, k}} \leq \mathrm{W} \mathrm{RT}_{n, k}^{m} \leq \mathrm{W} \mathrm{RT}_{n, k}^{*} \leq \mathrm{W} \mathrm{RT}_{n+1,2},
$$

in contradiction to Corollary 3.9. Hence, $\mathrm{RT}_{n, k}^{m+1} \leq \mathrm{W}_{\mathrm{W}} \mathrm{RT}_{n, k}^{m}$.

We note that Question 3.22 remains, whether additional factors can make up for color increases. The characterization given in Corollary 4.18 also implies the following result on the arithmetic complexity of homogeneous sets with the help of the uniform low basis theorem [3, Theorem 8.3].

Corollary 4.25 (Arithmetic complexity). Every computable sequence $\left(c_{i}\right)_{i}$ of colorings $c_{i}:[\mathbb{N}]^{n} \rightarrow k$ for $n \geq 1$ and $k \geq 2$ admits a sequence $\left(M_{i}\right)_{i}$ such that $\left\langle M_{0}, M_{1}, \ldots\right\rangle^{\prime} \leq_{\mathrm{T}} \emptyset^{(n+1)}$ and such that $M_{i}$ is an infinite homogeneous set for $c_{i}$ for each $i \in \mathbb{N}$.

Proof. We use the map $\mathrm{L}:=\mathrm{J}^{-1} \circ \lim$, where $\mathrm{J}: \mathbb{N}^{\mathbb{N}} \rightarrow \mathbb{N}^{\mathbb{N}}, p \mapsto p^{\prime}$ is the Turing jump operation. The uniform low basis theorem [3, Theorem 8.3] states that $\mathrm{WKL} \leq_{\mathrm{sW}} \mathrm{L}$ holds, which implies WKL ${ }^{(n)} \leq_{\mathrm{sW}} \mathrm{L}^{(n)}$, since jumps are monotone with respect to strong Weihrauch reducibility. By Corollary 4.18 we have $\widehat{\mathrm{RT}_{n, k}} \equiv_{\mathrm{W}} \mathrm{WKL}^{(n)}$, and hence any instance $\left(c_{i}\right)_{i}$ of $\widehat{\mathrm{RT}_{n, k}}$ has a solution $M=\left\langle M_{0}, M_{1}, \ldots\right\rangle$ whose jump $M^{\prime}$ is computable in $\emptyset^{(n+1)}$.

Here $n+1$ cannot be replaced by $n$ in $\emptyset^{(n+1)}$ by Corollary 4.18 , and in this sense this result is optimal. Hence, we have a striking difference in the arithmetic complexity between the non-uniform Ramsey theorem as witnessed by Theorems 1.3 and 1.6 and the uniform sequential version of Ramsey's theorem for sequences as witnessed by Corollary 4.25. This yields another proof of Corollary 3.10.

Since jumps commute with parallelization by [7, Proposition 5.7(3)], Corollary 4.18 yields also the following corollary, which states that under parallelization a jump of the colored versions of Ramsey's theorem corresponds exactly to an increase in cardinality by one.

Corollary 4.26 (Parallelized jumps). For all $n \geq 2, k \geq 2, \mathbb{N}$ we obtain

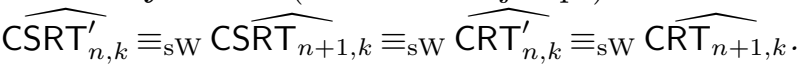

A similar property also holds for the uncolored version of Ramsey's theorem $\mathrm{RT}_{n, k}$, but in this case it cannot be so easily concluded since the parallelized uncolored theorem is not a cylinder by Corollary 3.16. Hence, we need the following reformulation of Corollary 4.10 that is justified by Lemma 4.9 
Corollary 4.27. $\mathrm{SRT}_{n+1, k} \leq_{\mathrm{W}} \mathrm{RT}_{n, k}^{\prime} \times \lim$ for all $n \geq 1, k \geq 1, \mathbb{N}$.

Now we are prepared to prove the following result on parallelized jumps of Ramsey's theorem.

Corollary 4.28 (Parallelized jumps). $\widehat{\mathrm{RT}_{n, k}^{\prime}} \equiv_{\mathrm{W}} \widehat{\mathrm{RT}_{n+1, k}}$ for all $n \geq 1$ and $k \geq 2, \mathbb{N}$.

Proof. It follows from Corollary 4.2 that $\mathrm{RT}_{n, k}^{\prime} \leq_{\mathrm{W}} \mathrm{RT}_{n+1, k}$, and hence one direction of the claims follows since parallelization is a closure operator. By Corollaries 4.18. 4.27 4.6. Fact 2.3 (3) and since parallelization commutes with products it follows that

$$
\mathrm{RT}_{n+1, k} \equiv_{\mathrm{W}} \widehat{\mathrm{SRT}_{n+1}, k} \leq \leq_{\mathrm{W}} \widehat{\mathrm{RT}_{n, k}^{\prime}} \times \lim \equiv_{\mathrm{W}} \widehat{\mathrm{RT}_{n, k}^{\prime}} \times \widehat{\lim _{\mathbb{N}}} \leq \mathrm{W} \widehat{\mathrm{RT}_{n, k}^{\prime}},
$$

which completes the proof.

We leave it open whether a corresponding fact can be established for the stable version of Ramsey's theorem (which is not the case for $n=1$ ). We have now completely provided all the positive information that is displayed in the diagram in Figure 2. What still remains to be done are some of the separations.

\section{RAMSEY's TheOREM FOR PAIRS}

In this section we want to discuss $\mathrm{RT}_{2,2}$, which is of particular interest. For one, we derive some conclusions from the general results that we proved before, we mention some results that have been proved by other authors, and we raise some open questions. The neighborhood of Ramsey's theorem in the Weihrauch lattice is illustrated in the diagram in Figure 4 .

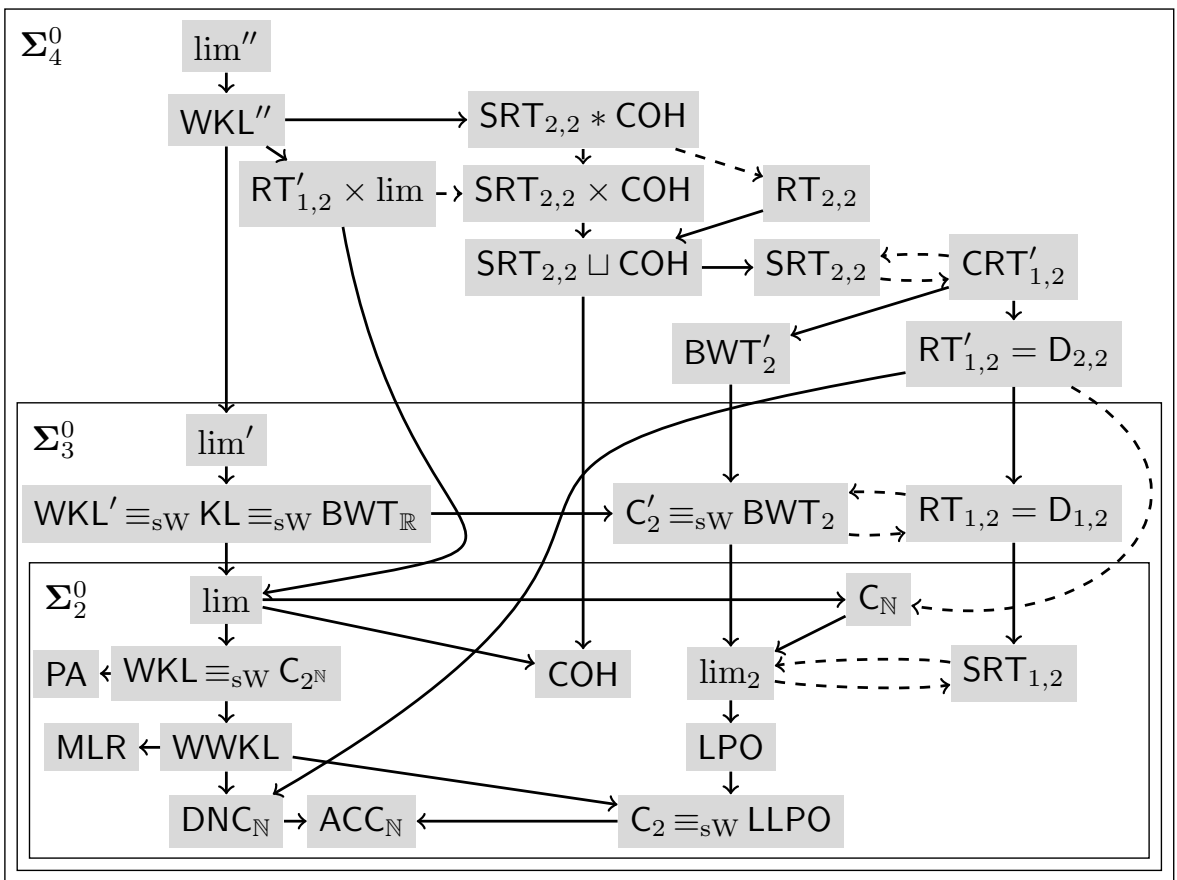

FiguRE 4. Ramsey's theorem for pairs and two colors in the Weihrauch lattice: all solid arrows indicate strong Weihrauch reductions against the direction of the arrow, all dashed arrows indicate ordinary Weihrauch reductions, and the boxes indicate the given levels of the effective Borel hierarchy. 
We start with two straightforward separation results that show that the jump of the cylindrification of $\mathrm{RT}_{1, k}$ (see Lemma 4.9) is incomparable with $\mathrm{RT}_{2,2}$.

Proposition 5.1. $\mathrm{RT}_{2,2} \not_{\mathrm{W}} \mathrm{RT}_{1, \mathbb{N}}^{\prime} \times \lim$ and $\mathrm{RT}_{1,2}^{\prime} \times \lim \not_{\mathrm{W}} \mathrm{RT}_{2, \mathbb{N}}$.

Proof. For every computable input $\mathrm{RT}_{1, \mathbb{N}}^{\prime} \times \lim$ clearly has a limit computable output, since for a limit computable coloring $c:[\mathbb{N}]^{1} \rightarrow \mathbb{N}$ there is always some $k \in \mathbb{N}$ such that $A:=\{i \in \mathbb{N}: c(i)=k\}$ is infinite. Such an $A$ is clearly limit computable and $A \in \mathrm{RT}_{1, \mathbb{N}}(c)$. However, $\mathrm{RT}_{2,2}$ has computable inputs $c:[\mathbb{N}]^{2} \rightarrow 2$, which have no limit computable outputs $A \in \mathrm{RT}_{2,2}(c)$ by Theorem 1.3 . Hence, $\mathrm{RT}_{2,2} \mathbb{E}_{\mathrm{W}} \mathrm{RT}_{1, \mathbb{N}}^{\prime} \times \lim$.

While $\lim \leq_{\mathrm{W}} \mathrm{RT}_{1,2}^{\prime} \times \lim$ holds obviously, we obtain $\lim \not_{\mathrm{W}} \mathrm{RT}_{2, \mathbb{N}}$ by Corollary 3.8 , Hence, $\mathrm{RT}_{1,2}^{\prime} \times \lim \not \mathrm{W}_{\mathrm{W}} \mathrm{RT}_{2, \mathbb{N}}$.

This result yields the following corollary.

Corollary 5.2. $\mathrm{RT}_{1,2}^{\prime} \times\left.\lim \right|_{\mathrm{W}} \mathrm{RT}_{2,2}$.

Since we have $\mathrm{SRT}_{2, \mathbb{N}} \leq_{\mathrm{W}} \mathrm{RT}_{1, \mathbb{N}} * \lim \equiv_{\mathrm{W}} \mathrm{RT}_{1, \mathbb{N}}^{\prime} \times \lim$ by Corollary 4.10 and Lemma 4.9, we also obtain the following corollary of Proposition 5.1

Corollary 5.3. $\mathrm{RT}_{2,2} \not_{\mathrm{W}} \mathrm{SRT}_{2, \mathbb{N}}$ and hence, in particular, $\mathrm{RT}_{2,2} \not \mathbb{W}_{\mathrm{W}} \mathrm{SRT}_{2,2}$.

The corresponding non-uniform result in reverse mathematics was much harder to obtain and solved a longstanding open question [15. The proof uses a non-standard model, whereas our result would follow from a separation with a standard model.

Among other things, we are going to discuss the relation of $\mathrm{RT}_{2,2}$ to the cohesiveness problem. The following proof is essentially the proof of Cholak, Jockusch and Slaman [12, Theorem 12.5].

Proposition 5.4 (Cohesiveness). $\mathrm{COH} \leq_{\mathrm{sW}} \mathrm{RT}_{2,2}$.

Proof. Let $\left(R_{i}\right)_{i}$ be a sequence of sets $R_{i} \subseteq \mathbb{N}$. We compute a sequence $\left(S_{i}\right)_{i}$ by $S_{2 i}:=R_{i}$ and $S_{2 i+1}:=\{i\}$ for all $i \in \mathbb{N}$. We let $d(i, j):=\min \left\{k: \chi_{S_{k}}(i) \neq \chi_{S_{k}}(j)\right\}$. The definition of $\left(S_{i}\right)_{i}$ ensures that $d$ is well-defined for all $i<j$, and it can be computed from $\left(S_{i}\right)_{i}$. We compute a coloring $c:[\mathbb{N}]^{2} \rightarrow 2$ with

$$
c\{i<j\}:=\left\{\begin{array}{ll}
0 & \text { if } i \in S_{d(i, j)} \\
1 & \text { otherwise }
\end{array},\right.
$$

and we consider an infinite homogeneous set $M \in \mathrm{RT}_{2,2}(c)$ and $i \in \mathbb{N}$. We claim that $M$ is cohesive for $\left(S_{i}\right)_{i}$ and hence for $\left(R_{i}\right)_{i}$. Let us assume for a contradiction that $k$ is the smallest number such that $M \cap S_{k}$ and $M \cap\left(\mathbb{N} \backslash S_{k}\right)$ are infinite. Since $k$ is minimal, there is a number $m \in \mathbb{N}$ such that $d(i, j) \geq k$ for all $i, j \geq m$ in $M$. There are also sufficiently large $i_{0}, i_{1}, i_{2} \geq m$ in $M$ such that $i_{0}<i_{1}<i_{2}, \chi_{S_{k}}\left(i_{0}\right) \neq \chi_{S_{k}}\left(i_{1}\right)$ and $\chi_{S_{k}}\left(i_{1}\right) \neq \chi_{S_{k}}\left(i_{2}\right)$. This implies $d\left(i_{0}, i_{1}\right)=k=d\left(i_{1}, i_{2}\right)$, which in turn yields $c\left\{i_{0}, i_{1}\right\} \neq c\left\{i_{1}, i_{2}\right\}$, in contradiction to the homogeneity of $M$. Hence, $M$ is cohesive for $\left(R_{i}\right)_{i}$, and we obtain $\mathrm{COH} \leq_{\mathrm{sW}} \mathrm{RT}_{2,2}$.

We obtain $\mathrm{RT}_{2,2} \not_{\mathrm{W}} \mathrm{COH}$ since $\mathrm{COH} \leq_{\mathrm{sW}} \lim$ by [8, Proposition 12.10$]$, but on the other hand $\mathrm{RT}_{2,2} \mathbb{Z}_{\mathrm{W}} \lim ^{\prime}$ by Corollary 4.20 . We obtain the following corollary.

Corollary 5.5. $\mathrm{SRT}_{2,2} \sqcup \mathrm{COH} \leq_{\mathrm{W}} \mathrm{RT}_{2,2} \leq_{\mathrm{W}} \mathrm{SRT}_{2,2} * \mathrm{COH} \leq_{\mathrm{sW}} \mathrm{WKL}^{\prime \prime}$.

Proof. By Proposition 5.4 Lemma 3.2 and since $\sqcup$ is the supremum with respect to $\leq_{\mathrm{W}}$ we have $\mathrm{SRT}_{2,2} \sqcup \mathrm{COH} \leq_{\mathrm{W}} \mathrm{RT}_{2,2}$. The reduction $\mathrm{RT}_{2,2} \leq_{\mathrm{W}} \mathrm{SRT}_{2,2}, * \mathrm{COH}$ follows from Proposition 4.11. The last mentioned reduction in the corollary follows from Corollary 4.15 and Facts 4.13 and 2.3 since

$$
\mathrm{SRT}_{2,2} * \mathrm{COH} \leq{ }_{\mathrm{W}} \mathrm{WKL}^{\prime \prime} * \mathrm{COH} \leq{ }_{\mathrm{W}} \mathrm{WKL}^{\prime} * \lim * \mathrm{COH} \equiv_{\mathrm{W}} \mathrm{WKL}^{\prime} * \mathrm{WKL}^{\prime} \equiv_{\mathrm{W}} \mathrm{WKL}^{\prime \prime}
$$


Given the fact that $\mathrm{SRT}_{2,2} \sqcup \mathrm{COH} \leq_{\mathrm{sW}} \mathrm{SRT}_{2,2} \times \mathrm{COH} \leq_{\mathrm{W}} \mathrm{SRT}_{2,2} * \mathrm{COH}$, it would be desirable to clarify the relation of $\mathrm{RT}_{2,2}$ to all the three mentioned problems.

Question 5.6. How does $\mathrm{RT}_{2,2}$ exactly relate to $\mathrm{SRT}_{2,2} \sqcup \mathrm{COH}, \mathrm{SRT}_{2,2} \times \mathrm{COH}$ and $\mathrm{SRT}_{2,2} * \mathrm{COH}$ ?

We can at least say something. We have $\mathrm{COH} \leq_{W} \lim$ by $[8$, Proposition 12.10] and by Corollary 4.10 and Lemma 4.9 we know that $\mathrm{SRT}_{2,2} \leq_{\mathrm{W}} \mathrm{RT}_{1,2}^{\prime} \times \lim$. Since lim is idempotent we obtain the following corollary.

Corollary 5.7. $\mathrm{SRT}_{2,2} \times \mathrm{COH} \leq_{\mathrm{W}} \mathrm{RT}_{1,2}^{\prime} \times \lim$.

With Proposition 5.1 we arrive at the following conclusion.

Corollary 5.8. $\mathrm{RT}_{2,2} \not \mathrm{W}_{\mathrm{W}} \mathrm{SRT}_{2,2} \times \mathrm{COH}$.

We also mention that Corollary 3.18 and Proposition 5.4 imply the following.

Corollary 5.9. $\mathrm{SRT}_{2,2} \times \mathrm{COH} \leq_{\mathrm{W}} \mathrm{RT}_{2,4}$.

We recall that the problem $R T_{1,2}^{\prime}$ is also studied under the name $D_{2}^{2}$ (see for instance [12, 14]). We introduce the following notation, where we use lower indices again.

Definition 5.10. We define $\mathrm{D}_{n, k}:=\mathrm{RT}_{1, k}^{(n-1)}$ for all $n, k \geq 1$.

Dzhafarov proved that $\mathrm{COH} \not_{\mathrm{sW}} \mathrm{D}_{2, k}$ holds for all $k \in \mathbb{N}$ [17, Corollary 1.10]. In fact, his key theorem [17, Theorem 1.5] yields even the following stronger result.

Corollary 5.11. $\mathrm{COH} \not \not_{\mathrm{sW}} \mathrm{CRT}_{1, k}^{(m)}$ for all $k, m \in \mathbb{N}$.

In a subsequent paper [18, Dzhafarov proved the following result.

Theorem 5.12. $\mathrm{COH} \not \underline{\mathrm{W}}_{\mathrm{W}} \mathrm{SRT}_{2,+}$ and hence, in particular, $\mathrm{COH} \not \underline{\mathrm{W}}_{\mathrm{SRT}} \mathrm{SR}_{2,2}$.

Since König's lemma KL is often discussed in the context of $\mathrm{RT}_{2,2}$, we mention it briefly in passing. By $K L: \subseteq \operatorname{Tr}_{\mathbb{N}} \rightrightarrows \mathbb{N}^{\mathbb{N}}$ we denote the multi-valued function that is defined on all infinite finitely branching trees $T \subseteq \mathbb{N}^{*}$ and such that $\operatorname{KL}(T)=[T]$ is the set of infinite paths of $T$. The set $\operatorname{Tr}_{\mathbb{N}}$ of trees $T \subseteq \mathbb{N}^{*}$ is represented via characteristic functions of such trees as usually. We can also consider a variant $K L_{+}$of $K L$, where the set of trees is represented by positive information only, i.e., by an enumeration of the corresponding tree $T$. The following theorem is essentially based on results from [7. If $n \in \mathbb{N}$, then we write $\widehat{n} \in \mathbb{N}^{\mathbb{N}}$ for the constant sequence with value $n$.

Theorem 5.13. $\mathrm{KL} \equiv_{\mathrm{sW}} \mathrm{KL}_{+} \equiv_{\mathrm{sW}} \mathrm{WKL}^{\prime}$.

Proof. It is clear that $\mathrm{KL} \leq_{\mathrm{sW}} \mathrm{KL}_{+}$. By [7, Corollaries 11.6 and 11.7] we have that $\mathrm{WKL}^{\prime} \equiv_{\mathrm{sW}} \mathrm{BWT}_{\mathbb{N}^{\mathrm{N}}}$.

It is easy to see that $\mathrm{KL}_{+} \leq_{\mathrm{sW}} \mathrm{BWT}_{\mathbb{N}^{\mathbb{N}}}$ : Given a finitely branching tree $T \subseteq \mathbb{N}^{*}$ by an enumeration $T=\left\{w_{n}: n \in \mathbb{N}\right\}$ we just compute a sequence $\left(x_{n}\right)_{n}$ in $\mathbb{N}^{\mathbb{N}}$ with $x_{n}:=w_{n} \widehat{0}$. Since $T$ is finitely branching, the set $\overline{\left\{x_{n}: n \in \mathbb{N}\right\}}$ is compact, and hence $\left(x_{n}\right)_{n}$ has cluster points. It is clear that all cluster points of $\left(x_{n}\right)_{n}$ are infinite paths of $T$. This proves $\mathrm{KL}_{+} \leq_{\mathrm{sW}} \mathrm{BWT}_{\mathbb{N}^{\mathrm{N}}}$.

We now prove BWT $\mathbb{N}^{\mathbb{N}} \leq_{s W} \mathrm{KL}_{+}$. Given a sequence $\left(x_{n}\right)_{n}$ that lies in a compact subset of $\mathbb{N}^{\mathbb{N}}$, we enumerate a tree $T$ as follows. We start with the empty tree $T$ and in step $n=0,1,2, \ldots$ we inspect $x_{n}$. If $w \sqsubseteq x_{n}$ is the longest prefix of $x_{n}$ that was already enumerated into $T$, then we enumerate $\left.x_{n}\right|_{k}$ into $T$ in step $n$, where $k=|w|+1$. In this way a tree $T$ is enumerated such that $[T]$ is the set of cluster points of $\left(x_{n}\right)_{n}$. The tree $T$ is a finitely branching tree since $\left(x_{n}\right)_{n}$ lies within a compact set. 
Finally, we prove $\mathrm{KL}_{+} \leq_{\mathrm{sW}} \mathrm{KL}$. Given a finitely branching tree $T \subseteq \mathbb{N}^{*}$ by an enumeration $T=\left\{w_{n}: n \in \mathbb{N}\right\}$, we compute a finitely branching tree $S \subseteq \mathbb{N}^{*}$ (by determining its characteristic function) such that $\operatorname{pr}_{1}([S])=[T]$, where

$$
\operatorname{pr}_{1}\left(\left\langle n_{0}, k_{0}\right\rangle,\left\langle n_{1}, k_{1}\right\rangle,\left\langle n_{2}, k_{2}\right\rangle \ldots\right)=\left(n_{0}, n_{1}, n_{2}, \ldots\right) \in \mathbb{N}^{\mathbb{N}}
$$

for all $n_{i}, k_{i} \in \mathbb{N}$. Since $\mathrm{pr}_{1}$ is computable, we obtain $\mathrm{KL}_{+} \leq_{\mathrm{sW}} \mathrm{KL}$ in this way. We create the tree $S$ in stages $n=0,1,2, \ldots$ and in stage $n$ we inspect the $n$-th word $w_{n}$ that is enumerated into $T$. In this stage we decide that a word of the form $u_{w, v}:=\left\langle w_{n}(0), v(0)\right\rangle\left\langle w_{n}(1), v(1)\right\rangle \ldots\left\langle w_{n}(k-1), v(k-1)\right\rangle$ with $k=\left|w_{n}\right|$ and all its prefixes belong to $S$, where $v$ is the lexicographically smallest word $v \in \mathbb{N}^{k}$ such that $u_{w, v}$ and all its prefixes have not yet been decided to belong to $\mathbb{N}^{*} \backslash S$. Additionally, we decide $w \in \mathbb{N}^{*} \backslash S$ for all non-empty words $w$ that have not yet been decided to belong to $S$ and such that $|w| \leq n$ and $w(i) \leq n$ for all $i \leq n-1$. On the one hand, this algorithm ensures that the tree $S$ is decided for larger and larger blocks of size $n$, and on the other hand, the construction guarantees that the resulting tree $S$ is finitely branching: on each level $i$ of $T$ only finitely many different values can occur and at some stage $n$ of the construction all words $w$ of length $i$ in $T$ have been considered and corresponding words $u_{w, v}$ have been added to $S$. The fact that we always choose the lexicographically smallest $v$ ensures that no new strings of length $i$ are added to $S$ after stage $n$. Finally, the construction also guarantees $\operatorname{pr}_{1}([S])=[T]$ as promised.

Now Theorem 5.13 and Corollary 3.30 yield the following result, which was independently proved in [23, Corollary 2.3] by a very different method.

Corollary 5.14. $\mathrm{KL} \leq_{\mathrm{W}} \mathrm{RT}_{3,2}$.

We note that by Corollary 3.15 the reduction cannot be replaced by a strong one since $\mathrm{C}_{2} \leq_{\mathrm{sW}} \mathrm{WKL} \leq_{\mathrm{sW}} \mathrm{KL}$. Likewise, one obtains WWKL $\not_{\mathrm{sW}} \mathrm{RT}_{n, k}$ for all $n, k \geq 1$ since $\mathrm{C}_{2} \leq_{\mathrm{sW}}$ WWKL. We note that by Corollary 3.9 we obtain that Corollary 5.14 is optimal in the following sense.

Corollary 5.15. $\mathrm{KL} \mathbb{L}_{\mathrm{W}} \mathrm{RT}_{2, \mathbb{N}}$.

However, we note the related Questions 3.31 and 4.7. Liu proved the following theorem and lemma [30, Theorem 1.5 and proof of Corollary 1.6].

Theorem 5.16 (Liu 2012 [30]). For any set $C$ not of PA-degree and any $A \subseteq \mathbb{N}$ there exists an infinite subset $G$ of $A$ or $\mathbb{N} \backslash A$ such that $G \oplus C$ is also not of PA-degree.

Lemma 5.17 (Liu 2012 [30]). For any set $C$ not of PA-degree and any uniformly $C$-computable sequence $\left(C_{i}\right)_{i}$ there exists a set $G$ which is cohesive for $\left(C_{i}\right)_{i}$ and such that $G \oplus C$ is also not of PA-degree.

We consider Peano arithmetic as the problem PA : $\mathcal{D} \rightrightarrows \mathcal{D}, \mathbf{b} \mapsto\{\mathbf{a}: \mathbf{a} \gg \mathbf{b}\}$ in the Weihrauch lattice, where $\mathcal{D}$ denotes the set of Turing degrees represented by their members and $\mathbf{a} \gg \mathbf{b}$ expresses the property that $\mathbf{a}$ is a PA-degree relative to b. From Liu's Theorem 5.16 we can directly derive PA $\not_{\mathrm{W}} \mathrm{D}_{n, 2}$ for all $n \geq 1$, and we also obtain the following corollary.

Corollary 5.18. PA $\not_{\mathrm{W}} \mathrm{CRT}_{1,2}^{(n-1)}$ for all $n \geq 1$.

In particular, this implies PA $\not_{\mathrm{W}} \mathrm{SRT}_{2,2}$, since $\mathrm{SRT}_{2,2} \equiv_{\mathrm{W}} \mathrm{CRT}_{1,2}^{\prime}$ by Theorem 4.3 , Using Liu's Lemma 5.17 this can be strengthened as follows.

Corollary 5.19. PA $\not_{\mathrm{W}} \mathrm{SRT}_{2,2} * \mathrm{COH}$.

Since $\mathrm{RT}_{2,2} \leq_{\mathrm{W}} \mathrm{SRT}_{2,2} * \mathrm{COH}$ by Proposition 4.11, this implies $\mathrm{PA} \leq_{\mathrm{W}} \mathrm{RT}_{2,2}$. Since $\mathrm{PA}<\mathrm{W}$ WKL [8, Theorem 5.5, Corollary 6.4], we also get the following conclusion. 
Corollary 5.20. WKL $\mathbb{E}_{\mathrm{W}} \mathrm{RT}_{2,2}$.

Likewise, one should be able to use the methods provided by Liu [31] to show that $M L R \not{ }_{W} R T_{2,2}$ (where MLR denotes the problem to find a point that is Martin-Löf random relative to the input) and hence WWKL $\mathbb{W}_{\mathrm{W}} \mathrm{RT}_{2,2}$. It would also be interesting to find out whether a variant of the above ideas can be used to answer Question 4.7 .

By $C C_{X}$ we denote the restriction of $C_{X}$ to connected subsets. We now want to discuss the intermediate value theorem IVT, which we do not define here, but we identify it with $\mathrm{CC}_{[0,1]}$ (see [10, Corollary 4.10] and [4, Theorem 6.2], where $\mathrm{CC}_{[0,1]}$ appears under the names $\mathrm{CC}_{1}$ and $\mathrm{C}_{I}$, respectively). $\mathrm{CC}_{[0,1]}$ can be seen as a one-dimensional version of the Brouwer fixed point theorem and thus of the equivalence class of WKL (see [10]). Hence, from the uniform perspective it is an obvious question to ask how IVT is related to Ramsey's theorem. In order to approach this question we provide a new upper bound on IVT which is of independent interest. By $\mathrm{CL}_{X}: \subseteq X^{\mathbb{N}} \rightrightarrows X$ we denote the cluster point problem of $X$, which is the problem to find a cluster point of the given input sequence (this is an extension of $\mathrm{BWT}_{X}$ since we do not demand that the input sequence lies within a compact set, but only that it admits a cluster point). By [7, Theorem 9.4] we have $\mathrm{CL}_{X} \equiv_{\mathrm{sW}} \mathrm{C}_{X}^{\prime}$ for every computable metric space $X$.

Proposition 5.21. IVT $\leq_{W} C_{\mathbb{N}}^{\prime}$.

Proof. We have IVT $\equiv_{\mathrm{W}} \mathrm{CC}_{[0,1]} \equiv_{\mathrm{W}} \mathrm{B}_{I}$ [4, Proposition 3.6 and Theorem 6.2], where $\mathrm{B}_{I}: \subseteq \mathbb{R}_{<} \times \mathbb{R}_{>} \rightrightarrows \mathbb{R},(x, y) \mapsto\{z: x \leq z \leq y\}$ with $\operatorname{dom}\left(\mathrm{B}_{I}\right):=\{(x, y): x \leq y\}$ denotes the boundedness problem introduced in [4] and $C_{\mathbb{N}}^{\prime} \equiv_{W} C L_{\mathbb{N}}[7$, Theorem 9.4]. Here $\mathbb{R}_{<}$and $\mathbb{R}_{>}$denote the real numbers represented as supremum and infimum of sequences of rational numbers, respectively. The aim is to prove $\mathrm{B}_{I} \leq_{W} \mathrm{CL}_{\mathbb{N}}$. Hence, we can assume that we have two monotone sequences $\left(a_{n}\right)_{n}$ and $\left(b_{n}\right)_{n}$ of rational numbers in [0,1] with $a_{n} \leq a_{n+1}<b_{n+1} \leq b_{n}$ for all $n \in \mathbb{N}$, and the goal is to find some $x \in[0,1]$ with $a:=\sup _{n \in \mathbb{N}} a_{n} \leq x \leq \inf _{n \in \mathbb{N}} b_{n}=: b$. Now we produce a sequence $\left(c_{n}\right)_{n}$ of natural numbers in stages $n=0,1,2, \ldots$ as follows, where we use a variable $k \in \mathbb{N}$ that is initially $k=0$. If at stage $n$ we find that $\left|b_{n}-a_{n}\right|<2^{-k-1}$, then we let $k:=k+1$, and we choose $c_{n}:=0$. Otherwise, we choose some value $c_{n} \neq 0$ such that $a_{n}<\overline{c_{n}}<b_{n}$, where $\overline{c_{n}}$ is the rational number with code $c_{n}$. If possible, we choose $c_{n}$ such that it is identical to the previous such number chosen, and if that is impossible, then we chose $c_{n}$ with $\overline{c_{n}}:=a_{n}+\frac{b_{n}-a_{n}}{2}$. Now there are two possible cases. If $a=b$, then the sequence $\left(c_{n}\right)_{n}$ contains infinitely many zeros and at most one other number $c$ occurs infinitely often in $\left(c_{n}\right)_{n}$ and it must be such that $a=b=\bar{c}$. Otherwise, if $a \neq b$, then the sequence $\left(c_{n}\right)_{n}$ contains only finitely many zeros, and only exactly one number $c$ different from zero occurs infinitely often. This number satisfies $a \leq \bar{c} \leq b$. The result of $\mathrm{CL}_{\mathbb{N}}\left(c_{n}\right)_{n}$ is one number $c$ that occurs infinitely often in $\left(c_{n}\right)_{n}$. If $c=0$, then we can compute $x:=a=b$ with the help of the input $\left(a_{n}\right)_{n},\left(b_{n}\right)_{n}$. If $c \neq 0$, then $x:=\bar{c}$ is a suitable result.

Propositions 5.21 7.2 and Theorem 7.9, which we are going to prove in Section 7 , yield the reduction IVT $\leq_{\mathrm{W}} \mathrm{SRT}_{2, \mathbb{N}}$. Ludovic Patey (personal communication) improved this result by showing that IVT $\leq_{W} R_{2,2}$. This answered an open question in an earlier version of this article.

$\mathrm{RT}_{2,2}$ and $\mathrm{SRT}_{2,2}$ cannot be Weihrauch reducible to any of PA, MLR or $\mathrm{COH}$ since the latter are reducible to lim and the former are not. However, we can even say more. We recall that a problem $f: \subseteq X \rightrightarrows Y$ is called densely realized, if the set $\{F(p): F \vdash f\}$ is dense in the domain of the representation of $Y$ for all $p$ that are names for an input in the domain of $f$, which intuitively means that a name of a result can start with any arbitrary prefix (that is a prefix of a valid name). Since it is easy to see that PA, MLR and $\mathrm{COH}$ are all densely realized, it follows by [8, Proposition 4.3] 
that $\mathrm{PA}^{(m)}, \mathrm{MLR}^{(m)}$ and $\mathrm{COH}^{(m)}$ are all indiscriminative. In particular we obtain the following corollary.

Corollary 5.22. $\mathrm{SRT}_{1,2} \not \mathrm{W}_{\mathrm{W}} \mathrm{PA}^{(m)}, \mathrm{SRT}_{1,2} \not \mathrm{EW}_{\mathrm{W}} \mathrm{MLR}^{(m)}$ and $\mathrm{SRT}_{1,2} \not \mathbb{W}_{\mathrm{W}} \mathrm{COH}^{(m)}$ for all $m \in \mathbb{N}$.

We mention that it was noted by Hirschfeldt and Jockusch [23, Figure 6] that the following corollary follows from [24, Theorem 2.3].

Corollary 5.23 (Diagonally non-computable functions). $\mathrm{DNC}_{\mathbb{N}} \leq_{\mathrm{sW}} \mathrm{RT}_{1,2}^{\prime}=\mathrm{D}_{2,2}$.

More information on the uniform content and relations between the problems DNC, PA, WKL, WWKL, MLR, COH and other problems can be found in 8$]$.

\section{Separation Techniques for Jumps}

In this section we plan to prove some further separation results related to $\mathrm{RT}_{2,2}$ (that justify some of the missing arrows in the diagram in Figure 4). For this purpose we collect some classical results that yield separation techniques for jumps. Often continuity arguments can be used for separation results. In the case of reductions to jumps of the form $f \leq_{\mathrm{W}} g^{\prime}$, such continuity arguments are not always applicable, since jumps $g^{\prime}$ implicitly involve limits. However, continuity arguments can occasionally be replaced by arguments based on the existence of continuity points, which are still available for limit computable functions. The classical techniques that we recall here concern $\boldsymbol{\Sigma}_{2}^{0}$-measurable functions, which form the topological counterpart of limit computable functions. Baire proved that the set of points of continuity of every $\boldsymbol{\Sigma}_{2}^{0}-$ measurable function is a comeager $G_{\delta}$-set under certain conditions. We recall that a set is called comeager if it contains a countable intersection of dense open sets. The following is result is [27, Theorem 24.14].

Proposition 6.1 (Baire 27). Let $X, Y$ be metric spaces, and let $Y$ additionally be separable. If $f: X \rightarrow Y$ is $\boldsymbol{\Sigma}_{2}^{0}-m e a s u r a b l e$, then the set of points of continuity of $f$ is a comeager $G_{\delta}$-set in $X$.

We recall that a topological space $X$ is called a Baire spac 4 , if every comeager set $C \subseteq X$ is dense in the space $X$. Hence we get the following corollary.

Corollary 6.2. Let $X$ and $Y$ be metric spaces, where $X$ is additionally a Baire space and $Y$ is separable. If $f: X \rightarrow Y$ is $\boldsymbol{\Sigma}_{2}^{0}$-measurable, then $\left.f\right|_{U}$ has a point of continuity for every non-empty open set $U \subseteq X$.

By the Baire category theorem [27, Theorem 8.4], every complete separable metric space is a Baire space. Moreover, closed subspaces of complete separable metric spaces are complete again. Hence Corollary 6.2 easily implies the direction "(1) $\Longrightarrow(2)$ " of the following characterization of $\boldsymbol{\Sigma}_{2}^{0}$-measurable functions [27, Theorem 24.15].

Theorem 6.3 (Baire characterization theorem [27]). Let $X, Y$ be separable metric spaces, and let $X$ additionally be complete. For every function $f: X \rightarrow Y$ the following conditions are equivalent to each other:

(1) $f$ is $\boldsymbol{\Sigma}_{2}^{0}$-measurable,

(2) $\left.f\right|_{A}$ has a point of continuity for every non-empty closed set $A \subseteq X$.

We note that while Theorem 6.3 yields a stronger conclusion than Corollary 6.2, it also requires stronger conditions on the domain $X$. In certain situations, we will be dealing with Baire spaces $X^{\mathbb{N}}$ which are not Polish spaces, and hence we will have to take recourse to Corollary 6.2 .

\footnotetext{
${ }^{8}$ We distinguish between $a$ Baire space $X$ in general and the Baire space $\mathbb{N}^{\mathbb{N}}$, in particular, which is also an instance of a Baire space.
} 
We recall [2, Proposition 9.1] that the limit map lim is a prototype of a $\boldsymbol{\Sigma}_{2}^{0}-$ measurable function (relatively to its domain of convergent sequences), and hence Theorem 6.3 can be used as a separation tool for certain reductions to jumps, since limit computable functions are closed under composition with continuous functions.

Fact 6.4. $\lim : \subseteq \mathbb{N}^{\mathbb{N}} \rightarrow \mathbb{N}^{\mathbb{N}}$ is $\boldsymbol{\Sigma}_{2}^{0}$-measurable relatively to its domain.

For the following result we use the Baire characterization theorem 6.3 as a separation technique. For partial functions $f: \subseteq X \rightarrow Y$ we use the notation $f(A):=$ $\{y \in Y:(\exists x \in A) f(x)=y\}$ for the image of $A$ under $f$.

Theorem 6.5. BWT $_{k+1} \not \underline{W}_{\mathrm{W}} \mathrm{RT}_{1, k}^{\prime} \times \lim$ for all $k \geq 1$.

Proof. Since $\mathrm{RT}_{1, k}^{\prime} \times \lim$ is a cylinder, it suffices to prove $\mathrm{BWT}_{k+1} \not_{\mathrm{sW}} \mathrm{RT}_{1, k}^{\prime} \times \lim$. Let us assume for a contradiction that $\mathrm{BWT}_{k+1} \leq_{\mathrm{sW}} \mathrm{RT}_{1, k}^{\prime} \times \lim$, i.e., there are computable functions $H, K$ such that $H G K$ is a realizer of $\mathrm{BWT}_{k+1}$ whenever $G$ is a realizer of $\mathrm{RT}_{1, k}^{\prime} \times \lim =\left(\mathrm{RT}_{1, k} \times \mathrm{id}\right) \circ(\lim \times \lim )$. Since $\mathrm{BWT}_{k+1}$ is total, it follows that $\left\langle K_{1}, K_{2}\right\rangle:=\langle\lim \times \lim \rangle \circ K$ is total too, and this function is $\boldsymbol{\Sigma}_{2}^{0}-$ measurable by Fact 2.2. We construct a number of points $p_{0}, \ldots, p_{k} \in \mathbb{N}^{\mathbb{N}}$ inductively. By the Theorem 6.3 there is a point $p_{0} \in \mathbb{N}^{\mathbb{N}}$ of continuity of $\left\langle K_{1}, K_{2}\right\rangle$. Let $\left\langle s_{0}, r_{0}\right\rangle:=\left\langle K_{1}\left(p_{0}\right), K_{2}\left(p_{0}\right)\right\rangle$, let $q_{0} \in \mathrm{RT}_{1, k}\left(s_{0}\right)$ be a maximal homogeneous set, and let $c_{0} \leq k-1$ be the corresponding color. Let $k_{0} \leq k$ be such that $H\left\langle q_{0}, r_{0}\right\rangle=k_{0}$. By continuity of $H$ there exists a prefix $w_{0} \sqsubseteq\left\langle q_{0}, r_{0}\right\rangle$ such that $H\left(w_{0} \mathbb{N}^{\mathbb{N}}\right)=\left\{k_{0}\right\}$. We can assume that $w_{0}=\left\langle x_{0}, y_{0}\right\rangle$ is of even length $2 n_{0}$. Since $\left\langle K_{1}, K_{2}\right\rangle$ is continuous at $p_{0}$, there is a $v_{0} \sqsubseteq p_{0}$ such that $\left.K_{1}\left(v_{0} \mathbb{N}^{\mathbb{N}}\right) \subseteq s_{0}\right|_{n_{0}} \mathbb{N}^{\mathbb{N}}$ and $K_{2}\left(v_{0} \mathbb{N}^{\mathbb{N}}\right) \subseteq y_{0} \mathbb{N}^{\mathbb{N}}$. Now we consider $N_{0}:=\{0, \ldots, k\} \backslash\left\{k_{0}\right\}$ and the closed set $A_{0}:=v_{0} N_{0}^{\mathbb{N}}$. Again by Theorem 6.3 there exists a point $p_{1} \in A_{0}$ of continuity of $\left.\left\langle K_{1}, K_{2}\right\rangle\right|_{A_{0}}$. Let $\left\langle s_{1}, r_{1}\right\rangle:=\left\langle K_{1}\left(p_{1}\right), K_{2}\left(p_{1}\right)\right\rangle$, let $q_{1} \in \mathrm{RT}_{1, k}\left(s_{1}\right)$ be a maximal homogeneous set, and let $c_{1} \leq k-1$ be the corresponding color. Let $k_{1} \leq k$ be such that $H\left\langle q_{1}, r_{1}\right\rangle=k_{1}$. Since $p_{1} \in A_{0}$ contains $k_{0}$ at most finitely many times, it follows that $k_{1} \neq k_{0}$. By continuity of $H$ there exists a prefix $w_{1} \sqsubseteq\left\langle q_{1}, r_{1}\right\rangle$ such that $H\left(w_{1} \mathbb{N}^{\mathbb{N}}\right)=\left\{k_{1}\right\}$. Again we can assume that $w_{1}=\left\langle x_{1}, y_{1}\right\rangle$ is of even length $2 n_{1}$, and additionally we can assume $n_{1}>n_{0}$. Since $v_{0} \sqsubseteq p_{1}$ we have $y_{0} \sqsubseteq K_{2}\left(p_{1}\right)=r_{1}$, and we obtain

$$
\left.s_{1}\right|_{n_{0}}=\left.K_{1}\left(p_{1}\right)\right|_{n_{0}}=\left.K_{1}\left(p_{0}\right)\right|_{n_{0}}=\left.s_{0}\right|_{n_{0}} .
$$

If $c_{1}=c_{0}$, i.e., if the maximal homogeneous sets $q_{0}$ and $q_{1}$ of $s_{0}$ and $s_{1}$, respectively, have the same color, then $x_{0}=\left.q_{0}\right|_{n_{0}}=\left.q_{1}\right|_{n_{0}}$ follows due to the maximality of $q_{0}$ and $q_{1}$ and hence $w_{0} \sqsubseteq\left\langle q_{1}, r_{1}\right\rangle$, which implies $H\left\langle q_{1}, r_{1}\right\rangle=k_{0}$ and hence $k_{0}=k_{1}$. Since $k_{0} \neq k_{1}$, we can conclude that $c_{0} \neq c_{1}$. Now we continue as before: since $\left.\left\langle K_{1}, K_{2}\right\rangle\right|_{A_{0}}$ is continuous at $p_{1}$, there is a $v_{1} \sqsubseteq p_{1}$ such that $\left.K_{1}\left(v_{1} \mathbb{N}^{\mathbb{N}}\right) \subseteq s_{1}\right|_{n_{1}} \mathbb{N}^{\mathbb{N}}$ and $K_{2}\left(v_{1} \mathbb{N}^{\mathbb{N}}\right) \subseteq y_{1} \mathbb{N}^{\mathbb{N}}$. Now we consider $N_{1}:=\{0, \ldots, k\} \backslash\left\{k_{0}, k_{1}\right\}$ and the closed set $A_{1}:=v_{1} N_{1}^{\mathbb{N}}$. Again by Theorem 6.3 there exists a point $p_{2} \in A_{1}$ of continuity of $\left\langle K_{1}, K_{2}\right\rangle_{A_{1}}$. In this case we eventually obtain colors $k_{2} \leq k, c_{2} \leq k-1$ such that $k_{2} \notin\left\{k_{0}, k_{1}\right\}$ and $c_{2} \notin\left\{c_{0}, c_{1}\right\}$. This construction can be repeated $k$ times since there are $k+1$ colors in $\{0, \ldots, k\}$ until $p_{0}, \ldots, p_{k}$ and $c_{0}, \ldots, c_{k}$ are determined. However, the construction yields that the $c_{0}, \ldots, c_{k}$ have to be pairwise different, which is a contradiction since there are only $k$ colors $c \in\{0, \ldots, k-1\}$ available.

We get the following immediate corollary with the help of Lemma 4.9 and Corollary 4.10

Corollary 6.6. $\mathrm{BWT}_{k+1} \not \mathrm{LW}_{\mathrm{W}} \mathrm{SRT}_{2, k}$ for all $k \geq 1$.

Since $\mathrm{BWT}_{k+1} \leq_{\mathrm{W}} \mathrm{RT}_{1, k+1} \leq_{\mathrm{W}} \mathrm{SRT}_{2, k+1}$ by Proposition 3.4 and Lemma 4.8, we obtain also the following separation result as a consequence of Corollary 6.6.

Corollary 6.7. $\mathrm{SRT}_{2, k+1} \not \leq \mathrm{W} \mathrm{SRT}_{2, k}$ for all $k \geq 1$. 
As next separation result we want to prove $B \mathrm{BT}_{2}^{\prime} \mathbb{Z}_{\mathrm{W}} \mathrm{RT}_{1,2}^{\prime}$. To this end we are going to use Corollary 6.2 as a separation tool. It is not too difficult to see that for every topological space $X$ that is endowed with the discrete topology, the product space $X^{\mathbb{N}}$ is a Baire space. We will apply this observation to the particular case of the set

$$
X_{2}:=\left\{p \in 2^{\mathbb{N}}:(\exists k)(\forall n \geq k) p(n)=p(k)\right\}
$$

of convergent sequences of zeros and ones. On the other hand, $X_{2}$ is also endowed with the subspace topology that it inherits from the ordinary product topology on $\mathbb{N}^{\mathbb{N}}$. In both cases, we assume that $X_{2}^{\mathbb{N}}$ is equipped with the respective product topology. In order to distinguish both topologies, we speak about "discrete continuity" of a map $f: X_{2}^{\mathbb{N}} \rightarrow Y$, if $X_{2}$ is endowed with the discrete topology and about continuity if the usual subspace topology of $\mathbb{N}^{\mathbb{N}}$ is used for $X_{2}$. In the latter case, the map

$$
h_{2}: X_{2}^{\mathbb{N}} \rightarrow \mathbb{N}^{\mathbb{N}},\left(\left(x_{i, j}\right)_{i}\right)_{j} \mapsto\left\langle\left(x_{0, j}\right)_{j},\left(x_{1, j}\right)_{j},\left(x_{2, j}\right)_{j}, \ldots\right\rangle,
$$

is a computable embedding, i.e., $h_{2}$ as well as the partial inverses $h_{2}^{-1}$ are both computable and continuous. Intuitively, $h_{2}$ swaps rows and columns and applies the Cantor encoding $\langle\ldots\rangle\rangle$, both being purely technical changes. Hence we will identify $X_{2}^{\mathbb{N}}$ with (a subspace of) $\mathbb{N}^{\mathbb{N}}$ via $h_{2}$ in the following proof. We note that $\mathrm{BWT}_{2}^{\prime}$ formally has the same domain as $\mathrm{BWT}_{2}$, namely $2^{\mathbb{N}}$. But a realizer of $\mathrm{BWT}_{2}^{\prime}$ has to use $\lim _{2^{\mathrm{N}}}$ as input representation and hence it will have domain $h_{2}\left(X_{2}^{\mathbb{N}}\right)$, which we identify with $X_{2}^{\mathbb{N}}$. For $w \in \mathbb{N}^{n}$ and $p \in \mathbb{N}^{\mathbb{N}}$ we use the notation $w^{\rightarrow} p:=w p(n) p(n+1) p(n+2) \ldots$ for the sequence that is obtained from $p$ by replacing the first $n$ positions of $p$ by $w$.

Theorem 6.8. $\mathrm{BWT}_{2}^{\prime} \not_{\mathrm{W}} \mathrm{RT}_{1,2}^{\prime}$.

Proof. Let us assume for a contradiction that $\mathrm{BWT}_{2}^{\prime} \leq_{\mathrm{W}} \mathrm{RT}_{1,2}^{\prime}$. Then there are computable functions $H, K$ such that $H\langle\mathrm{id}, G K\rangle$ is a realizer of $\mathrm{BWT}_{2}^{\prime}$ whenever $G$ is a realizer of $\mathrm{RT}_{1,2}^{\prime}$. We tacitly identify $X_{2}^{\mathbb{N}}$ with a subspace of $\mathbb{N}^{\mathbb{N}}$ via $h_{2}$. Since $\mathrm{BWT}_{2}^{\prime}: 2^{\mathbb{N}} \rightrightarrows\{0,1\}$ is total, it follows that $\lim K: X_{2}^{\mathbb{N}} \rightarrow \mathbb{N}^{\mathbb{N}}$ is also total and $\boldsymbol{\Sigma}_{2}^{0}$ measurable. If we endow $X_{2}$ with the discrete topology, then $X_{2}^{\mathbb{N}}$ is a Baire space, and by Corollary 6.2 there exists a point of discrete continuity $x \in X_{2}^{\mathbb{N}}$ of $\lim K$. Let $c:=\lim K(x)$ be the corresponding coloring. Then there is some maximal infinite homogeneous set $q \in \mathrm{RT}_{1,2}^{\prime}(c)$, and there is a realizer $G$ of $\mathrm{RT}_{1,2}^{\prime}$ that maps $K(x)$ to $q$. By continuity of $H$ there is some $m \in \mathbb{N}$ such that $H\left\langle\left. x\right|_{m} X_{2}^{\mathbb{N}},\left.q\right|_{m} \mathbb{N}^{\mathbb{N}}\right\rangle=\{e\}$ for some $e \in\{0,1\}$. Without loss of generality, we assume $e=0$. Hence, by discrete continuity of $\lim K$ at $x$, there is some $k>m$ such that $\left.\lim K\left(\left.x\right|_{k} X_{2}^{\mathbb{N}}\right) \subseteq c\right|_{m} 2^{\mathbb{N}}$.

We construct some $y=\left(\left.x\right|_{k}, z_{0}, z_{1}, z_{2} \ldots\right) \in X_{2}^{\mathbb{N}}$ such that all $z_{i} \in X_{2}$ converge to 1 , and the coloring $c_{y}:=\lim K(y)$ has maximal homogeneous sets $q_{0}, q_{1} \in \mathrm{RT}_{1,2}\left(c_{y}\right)$ of different colors. This yields a contradiction: since $\left.x\right|_{k} \sqsubseteq y$, we obtain $\left.c\right|_{m} \sqsubseteq c_{y}$, and hence $\left.q\right|_{m}$ must be prefix of either $q_{0}$ or $q_{1}$, say $q_{0}$, and this implies $H\left\langle y, q_{0}\right\rangle=e=0$, whereas the correct result would have to be 1 , since all the $z_{i}$ converge to 1 . We describe the inductive construction of $y$ in even and odd stages $i \in \mathbb{N}$.

Stage 0 : Let $y_{0}:=\left.x\right|_{k} \widehat{0} \widehat{0} \ldots, c_{0}:=\lim K\left(y_{0}\right)$, and let $r_{0} \in \mathrm{RT}_{1,2}\left(c_{0}\right)$. Then by continuity of $H$ there is some $m_{0}>k$ such that $H\left\langle\left. x\right|_{k} w_{m_{0}} X_{2}^{\mathbb{N}},\left.r_{0}\right|_{m_{0}} \mathbb{N}^{\mathbb{N}}\right\rangle=\{0\}$ with $w_{m}:=\left(0^{m} \widehat{1}\right)^{m}=\left(0^{m} \widehat{1}, 0^{m} \widehat{1}, \ldots, 0^{m} \widehat{1}\right) \in X_{2}^{m}$ for all $m \in \mathbb{N}$.

Stage 1: Let $y_{1}:=\left.x\right|_{k} w_{m_{0}} \hat{1} \hat{1} \ldots, c_{1}:=\lim K\left(y_{1}\right)$, and let $r_{1} \in \mathrm{RT}_{1,2}\left(c_{1}\right)$. Then also $0^{m_{0} \rightarrow} r_{1} \in \mathrm{RT}_{1,2}\left(c_{1}\right)$, and by continuity of $H$ there is some $m_{1}>k+m_{0}$ such that $H\left\langle\left. y_{1}\right|_{m_{1}} X_{2}^{\mathbb{N}},\left.0^{m_{0} \rightarrow} r_{1}\right|_{m_{1}} \mathbb{N}^{\mathbb{N}}\right\rangle=\{1\}$.

We now assume that $i \geq 1$ and that $y_{0}, \ldots, y_{2 i-1} \in X_{2}^{\mathbb{N}}$ and $m_{0}, \ldots, m_{2 i+1} \in \mathbb{N}$ have already been constructed.

Stage $2 i$ : Let $y_{2 i}:=\left.y_{2 i-1}\right|_{m_{2 i-1}} \widehat{0} \widehat{0} \ldots, c_{2 i}:=\lim K\left(y_{2 i}\right)$, and let $r_{2 i} \in \mathrm{RT}_{1,2}\left(c_{2 i}\right)$. Then by continuity of $H$ there is some $m_{2 i}>m_{2 i-1}$ such that

$$
H\left\langle\left. y_{2 i-1}\right|_{m_{2 i-1}} w_{m_{2 i}} X_{2}^{\mathbb{N}},\left.0^{m_{2 i-1} \rightarrow} r_{2 i}\right|_{m_{2 i}} \mathbb{N}^{\mathbb{N}}\right\rangle=\{0\} .
$$


Stage $2 i+1$ : Let $y_{2 i+1}:=\left.y_{2 i-1}\right|_{m_{2 i-1}} w_{m_{2 i}} \widehat{1} \widehat{1} \ldots, c_{2 i+1}:=\lim K\left(y_{2 i+1}\right)$, and let $r_{2 i+1} \in \mathrm{RT}_{1,2}\left(c_{2 i+1}\right)$. Then also $0^{m_{2 i} \rightarrow} r_{2 i+1} \in \mathrm{RT}_{1,2}\left(c_{2 i+1}\right)$, and by continuity of $H$ there is some $m_{2 i+1}>m_{2 i-1}+m_{2 i}$ such that

$$
H\left\langle\left. y_{2 i+1}\right|_{m_{2 i+1}} X_{2}^{\mathbb{N}},\left.0^{m_{2 i} \rightarrow} r_{2 i+1}\right|_{m_{2 i+1}} \mathbb{N}^{\mathbb{N}}\right\rangle=\{1\} .
$$

This construction yields a sequence $\left(y_{i}\right)_{i}$ in $X_{2}^{\mathbb{N}}$ and a strictly increasing sequence $\left(m_{i}\right)_{i}$ in $\mathbb{N}$ such that $\left.y_{2 i-1}\right|_{m_{2 i-1}} \sqsubseteq y_{2 i+1}$ for all $i$. Since all the blocks $w_{m}$ that are added to the initial $\left.x\right|_{k}$ in the odd stages are of the form $w_{m}=\left(z_{1}, \ldots, z_{m}\right)$, with $z_{i} \in X_{2}$ that all converge to 1 , it follows that $\left(y_{2 i+1}\right)_{i}$ converges to a $y=$ $\left(\left.x\right|_{k}, z_{0}, z_{1}, z_{2} \ldots\right) \in X_{2}^{\mathbb{N}}$, with $z_{i}$ that all converge to 1 . Let $c_{y}:=\lim K(y)$ be the corresponding coloring. We now prove that there are homogeneous sets in $\mathrm{RT}_{1,2}\left(c_{y}\right)$ of different color. We fix some maximal homogeneous set $r \in \mathrm{RT}_{1,2}\left(c_{y}\right)$. It suffices to prove that there are infinitely many $i$ with $r(i)=0$. The inductive proof follows the stages of the construction above and uses the corresponding objects constructed therein.

Stage 0,1: We obtain $\left.r_{0}\right|_{m_{0}} \nsubseteq r$, since otherwise there is some $s \in \mathrm{RT}_{1,2}\left(c_{y}\right)$ such that $\left.r_{0}\right|_{m_{0}} \sqsubseteq s$ and $H\langle y, s\rangle=1$. Since $\left.x\right|_{k} w_{m_{0}} \sqsubseteq y$ this contradicts the choice of $m_{0}$ in Stage 0. In particular, we obtain that there is some $i$ with $0 \leq i<m_{0}$ and $r(i)=0$.

Stages $2 i, 2 i+1$ : Likewise, $\left.0^{m_{2 i-1}} \rightarrow r_{2 i}\right|_{m_{2 i}} \nsubseteq r$, since otherwise there is some $s \in$ $\mathrm{RT}_{1,2}\left(c_{y}\right)$ such that $\left.0^{m_{2 i-1}} \rightarrow r_{2 i}\right|_{m_{2 i}} \sqsubseteq s$ and $H\langle y, s\rangle=1$. Since $\left.y_{2 i-1}\right|_{m_{2 i-1}} w_{m_{2 i}} \sqsubseteq y$ this contradicts the choice of $m_{2 i}$ in Stage $2 i$. In particular, there is some $i$ with $m_{2 i-1} \leq i<m_{2 i}$ and $r(i)=0$.

Altogether, this proves that there are infinitely many $i$ with $r(i)=0$, since $\left(m_{i}\right)_{i}$ is strictly increasing.

By Corollary 4.6 we have $\lim _{\mathbb{N}} \leq_{\mathrm{W}} \mathrm{RT}_{1,2}^{\prime}$. On the other hand, we obtain the following corollary.

Fact 6.9. $\lim _{\mathbb{N}} \not_{\mathrm{W}} \mathrm{BWT}_{k}^{(m)}$ for all $k, m \geq 1$.

Proof. Let us denote by $\mathrm{UC}_{\mathbb{N}}$ the restriction of $\mathrm{C}_{\mathbb{N}}$ to singletons. By [7, Fact 3.2(1)] $U C_{\mathbb{N}}$ is a strong fractal, and $C_{\mathbb{N}}$ is obviously slim, which means that range $\left(\mathrm{UC}_{\mathbb{N}}\right)=$ range $\left(C_{\mathbb{N}}\right)$. Under these conditions [7, Theorem 13.3] yields $C_{\mathbb{N}} \not \mathbb{W}_{\mathrm{W}} \mathrm{BWT}_{k}^{(m)}$ since $\left|\operatorname{range}\left(C_{\mathbb{N}}\right)\right|=|\mathbb{N}|>k=\left|\operatorname{range}\left(\mathrm{BWT}_{k}^{(m)}\right)\right|$. By [7, Proposition 3.8] we have $\lim _{\mathbb{N}} \equiv_{\mathrm{W}} \mathrm{C}_{\mathbb{N}}$, which implies the claim.

We could also replace the application of [7, Theorem 13.3] in this proof by an application of Proposition 7.3 that we prove below. Now it follows with Fact 6.9 that $\mathrm{RT}_{1,2}^{\prime} \leq_{\mathrm{W}} \mathrm{BWT}_{2}^{\prime}$. Hence, together with Theorem 6.8 we obtain the following corollary.

Corollary 6.10. BWT $\left.{ }_{2}^{\prime}\right|_{\mathrm{W}} \mathrm{RT}_{1,2}^{\prime}$.

By Proposition 3.4 we have $\mathrm{BWT}_{2} \equiv_{\mathrm{W}} \mathrm{RT}_{1,2}$, but Corollary 6.10 shows that the situation is very different for strong Weihrauch reducibility. With the help of [7, Proposition 5.6(2)] we obtain the following.

Corollary 6.11. $\left.\mathrm{BWT}_{2}\right|_{\mathrm{sW}} \mathrm{RT}_{1,2}$.

It is clear that $\mathrm{D}_{2,2}=\mathrm{RT}_{1,2}^{\prime} \leq_{\mathrm{sW}} \mathrm{CRT}_{1,2}^{\prime} \equiv_{\mathrm{W}} \mathrm{SRT}_{2,2}$ by Theorem 4.3, and by Theorem 3.5 we also obtain $\mathrm{BWT}_{2}^{\prime} \leq_{\mathrm{sW}} \mathrm{CRT}_{1,2}^{\prime}$. Now Corollary 6.10 also leads to the following separation result which was independently proved by Dzhafarov [18, Corollary 3.3].

Corollary 6.12. SRT $T_{2,2} \not \underline{W}_{\mathrm{W}} \mathrm{D}_{2,2}$.

Altogether, we have now separated several of the degrees illustrated in the diagram of Figure 4 


\section{Boundedness AND InduCtion}

The purpose of this section is to collect some results on how Ramsey's theorem is related to boundedness and induction. In reverse mathematics there is a well-known strict hierarchy of induction principles $\mathrm{I} \Sigma_{n}^{0}$ and boundedness principles $\mathrm{B} \Sigma_{n}^{0}$ (see [22, Theorem 4.32]):

$$
\mathrm{B} \Sigma_{1}^{0} \leftarrow \mathrm{I} \Sigma_{1}^{0} \leftarrow \mathrm{B} \Sigma_{2}^{0} \leftarrow \mathrm{I} \Sigma_{2}^{0} \leftarrow \ldots
$$

While these principles have no immediate interpretation as problems in the Weihrauch lattice, there are equivalent formulations of these principles that do have such interpretations. For instance, it is well-known that $\mid \Sigma_{n}^{0}$ is equivalent to the least number principle $\mathrm{L} \Pi_{n}^{0}$ over a very weak system [21, Theorem 2.4]. The least number principle $\mathrm{L} \Pi_{1}^{0}$ can be interpreted as the the following problem:

$$
\min : \subseteq \mathbb{N}^{\mathbb{N}} \rightarrow \mathbb{N}, p \mapsto \min \{n \in \mathbb{N}:(\forall k \in \mathbb{N}) p(k) \neq n\}
$$

Equivalently, one could also consider the map $\min : \subseteq \mathcal{A}_{-}(\mathbb{N}) \rightarrow \mathbb{N}, A \mapsto \min (A)$ that maps every non-empty closed set $A \subseteq \mathbb{N}$ (given by an enumeration of its complement) to its minimum. The $n$-th jump $\min ^{(n)}$ now clearly corresponds to $\mathrm{L}_{n+1}^{0}$ for all $n \in \mathbb{N}$. The following result is easy to obtain.

Proposition 7.1 (Least number principle). $\mathrm{C}_{\mathbb{N}} \equiv_{\mathrm{sW}} \min$.

Proof. $\mathrm{C}_{\mathbb{N}} \leq_{\mathrm{sW}}$ min is obvious, since given an enumeration $p$ of a the complement of a non-empty set $A \subseteq \mathbb{N}$, we have $\min (p) \in A$. We also have $\min \leq_{\mathrm{sW}} \mathrm{C}_{\mathbb{N}}$ : Given a sequence $p \in \mathbb{N}^{\mathbb{N}}$ we start enumerating the complement of a set $A \subseteq \mathbb{N}$ while we inspect the sequence $p$. The algorithm proceeds in stages $i=0,1,2, \ldots$ In stage $i$ we let $k_{i}:=\min \{n \in \mathbb{N}:(\forall j \leq i) p(j) \neq n\}$ and we remove all $\langle n, m\rangle$ from $A$ for $n, m \leq i$ and $m \neq k_{i}$. Hence, the final set $A$ satisfies

$$
A \subseteq\{\langle n, k\rangle \in \mathbb{N}: n \in \mathbb{N}, k=\min (\mathbb{N} \backslash \operatorname{range}(p))\}
$$

and given a number $\langle n, k\rangle \in A$ we can easily extract $k=\min (\mathbb{N} \backslash \operatorname{range}(p))$. Hence, $\min \leq_{\mathrm{sW}} \mathrm{C}_{\mathbb{N}}$.

Since jumps preserve strong Weihrauch equivalences we obtain $C_{\mathbb{N}}^{(n)} \equiv_{\mathrm{sW}} \min { }^{(n)}$ for all $n \in \mathbb{N}$, and hence $\mathrm{C}_{\mathbb{N}}^{(n)}$ can be seen as a counterpart of $I \Sigma_{n+1}^{0}$ for all $n \in \mathbb{N}$.

Likewise, the boundedness principle $\mathrm{B} \Sigma_{n}^{0}$ has been characterized with the help of the pigeonhole principle and the regularity principle $\mathrm{R} \Sigma_{n}^{0}$. More precisely, it is known that $\mathrm{B} \Sigma_{n+2}^{0}$ is equivalent to $\mathrm{R} \Sigma_{n+1}^{0}$ for all $n \in \mathbb{N}$ over a very weak system [21, Theorem 2.23]. Now $B W T_{\mathbb{N}}$ can be seen as a counterpart of $\mathrm{R} \Sigma_{1}^{0}$ since the computational goal of $\mathrm{BWT}_{\mathbb{N}}$ is to find a number that appears infinitely often in a bounded sequence. More generally, $\mathrm{R} \Sigma_{n+1}^{0}$ corresponds to $\mathrm{BWT}_{\mathbb{N}}^{(n)}$ for all $n \in \mathbb{N}$. We recall that $\mathrm{K}_{\mathbb{N}}$ denotes compact choice on $\mathbb{N}$, which can be defined as closed choice $C_{\mathbb{N}}$, except that additionally an upper bound for the input set is provided (see [7] for a precise definition). Another characterization of $\mathrm{K}_{\mathbb{N}}$ is $\mathrm{K}_{\mathbb{N}} \equiv_{\mathrm{sW}} \mathrm{C}_{2}^{*}$ (which holds by [7, Proposition 10.9] where LLPO $\equiv_{\mathrm{sW}} \mathrm{C}_{2}$ ) and we obtain $\mathrm{K}_{\mathbb{N}}^{\prime} \equiv_{\mathrm{sW}} \mathrm{BWT}_{\mathbb{N}}$ by [7, Corollary 11.10]. Hence, it is justified to say that $\mathrm{K}_{\mathbb{N}}^{(n+1)}$ corresponds to $\mathrm{B} \Sigma_{n+2}^{0}$ for all $n \in \mathbb{N}$.

Analogously to the above implication chain for $I \Sigma_{n}^{0}$ and $\mathrm{B} \Sigma_{n}^{0}$ we obtain

$$
\mathrm{K}_{\mathbb{N}}<{ }_{\mathrm{W}} \mathrm{C}_{\mathbb{N}}<{ }_{\mathrm{W}} \mathrm{K}_{\mathbb{N}}^{\prime}<{ }_{\mathrm{W}} \mathrm{C}_{\mathbb{N}}^{\prime}<\mathrm{W} \mathrm{K}_{\mathbb{N}}^{\prime \prime}<_{\mathrm{W}} \mathrm{C}_{\mathbb{N}}^{\prime \prime}<\mathrm{W} \ldots
$$

This reduction chain is based on the following observation.

Proposition 7.2. $\mathrm{K}_{\mathbb{N}}^{(n)}<_{\mathrm{sW}} \mathrm{C}_{\mathbb{N}}^{(n)}<_{\mathrm{sW}} \mathrm{K}_{\mathbb{N}}^{(n+1)}$ and $\mathrm{K}_{\mathbb{N}}^{(n)}<_{\mathrm{W}} \mathrm{C}_{\mathbb{N}}^{(n)}<_{\mathrm{W}} \mathrm{K}_{\mathbb{N}}^{(n+1)}$ for $n \in \mathbb{N}$.

Proof. We obtain $\mathrm{K}_{\mathbb{N}} \leq_{\mathrm{sW}} \mathrm{C}_{\mathbb{N}} \equiv_{\mathrm{sW}} \lim _{\mathbb{N}} \leq_{\mathrm{sW}} B \mathrm{BWT}_{\mathbb{N}} \equiv_{\mathrm{sW}} \mathrm{K}_{\mathbb{N}}^{\prime}$. Here $\mathrm{C}_{\mathbb{N}} \equiv_{\mathrm{sW}} \lim _{\mathbb{N}}$ holds by [7. Proposition 3.8]. The claimed reductions follow since jumps are monotone with respect to $\leq_{\mathrm{sW}}$. The separation results follow from Fact 2.3 (5) since we 
have $\widehat{\mathrm{C}_{\mathbb{N}}} \equiv_{\mathrm{sW}} \widehat{\lim _{\mathbb{N}}} \equiv_{\mathrm{sW}} \lim$ (see also [3, Example 3.10]), $\widehat{\mathrm{K}_{\mathbb{N}}} \equiv_{\mathrm{sW}} \mathrm{WKL}$ and since parallelization commutes with jumps. Here $\widehat{\mathrm{K}_{\mathbb{N}}} \equiv_{\mathrm{sW}} \mathrm{WKL}$ holds since we obtain that $\mathrm{C}_{2} \leq_{\mathrm{sW}} \mathrm{C}_{2}^{*} \equiv_{\mathrm{sW}} \mathrm{K}_{\mathbb{N}} \leq_{\mathrm{sW}} \widehat{\mathrm{C}_{2}} \equiv_{\mathrm{sW}} \mathrm{WKL}$ and parallelization is a closure operator.

In reverse mathematics a considerable amount of work has been spent in order to calibrate Ramsey's theorem for pairs according to the above boundedness and induction principles. Hence, it is natural to ask how it compares uniformly to choice principles. We start with some easy observations, and as a preparation we prove the following result. The proof is a simple variation of the proof of [7, Theorem 13.3].

Proposition 7.3 (Choice and cardinality). Let $f: \subseteq X \rightrightarrows \mathbb{N}$ and $X \subseteq \mathbb{N}$ be such that $\mathrm{C}_{X} \leq_{\mathrm{w}} f$. Then $|X| \leq|\operatorname{range}(f)|$.

Proof. Let us assume that $\mathrm{C}_{X} \leq_{\mathrm{W}} f$. We use the representation $\psi_{\text {- of closed subsets }}$ of $X$. Then there are computable $H, K$ such that $H\langle$ id, $G K\rangle \vdash \mathrm{C}_{X}$ for all $G \vdash f$. We fix some realizer $G \vdash f$. For simplicity and without loss of generality we assume that $G$ and $H$ have target space $\mathbb{N}$. We consider the following claim: for each $i \in \mathbb{N}$ with $|X|>i$ there exist

(1) $k_{i} \in X \backslash\left\{k_{0}, \ldots, k_{i-1}\right\}$,

(2) $n_{i} \in \operatorname{range}(f) \backslash\left\{n_{0}, \ldots, n_{i-1}\right\}$,

(3) $p_{i}$, a name of a closed subset $A_{i} \subseteq X$,

(4) $w_{i} \sqsubseteq p_{i}$,

such that $w_{i-1} \sqsubseteq w_{i}, G K\left(p_{i}\right)=n_{i}$ and $H\left\langle w_{i} \mathbb{N}^{\mathbb{N}}, n_{i}\right\rangle=k_{i}$. Let $w_{-1}$ be the empty word. We prove this claim by induction on $i$. Let us assume that $|X|>0$, and let $p_{0}$ be a name of $A_{0}:=X$. Then $k_{0}:=H\left\langle p_{0}, G K\left(p_{0}\right)\right\rangle \in A_{0}$ and $n_{0}:=G K\left(p_{0}\right)$. By continuity of $H$ there is some $w_{0} \sqsubseteq p_{0}$ such that such that $H\left\langle w_{0} \mathbb{N}^{\mathbb{N}}, n_{0}\right\rangle=k_{0}$. Let us now assume that $|X|>1$, and let $p_{1}$ be a name of $A_{1}:=X \backslash\left\{k_{0}\right\}$ with $w_{0} \sqsubseteq p_{1}$. Then $k_{1}:=H\left\langle p_{1}, G K\left(p_{1}\right)\right\rangle \in A_{1}$ and $n_{1}:=G K\left(p_{1}\right)$. Since $k_{1} \neq k_{0}$, we obtain $n_{0} \neq n_{1}$ since $H\left\langle w_{0} \mathbb{N}^{\mathbb{N}}, n_{0}\right\rangle=k_{0}$. By continuity of $H$ there is some $w_{1} \sqsubseteq p_{1}$ with $w_{0} \sqsubseteq w_{1}$ such that $H\left\langle w_{1} \mathbb{N}^{\mathbb{N}}, n_{1}\right\rangle=k_{1}$. The proof can now continue inductively as above which proves the claim. The claim implies $|X| \leq|\operatorname{range}(f)|$.

From this result we can conclude the following observation.

Proposition 7.4 (Finite Choice). $\mathrm{C}_{k} \leq_{\mathrm{W}} \mathrm{SRT}_{1, k}$ and $\mathrm{C}_{k+1} \not_{\mathrm{W}} \mathrm{RT}_{1, k}$ for all $k \geq 1$.

Proof. By Lemma 3.4 we have $\lim _{k} \equiv_{\mathrm{W}} \mathrm{SRT}_{1, k}$, hence the first reduction follows from 7. Corollary 13.8] and can easily been proved directly. By Lemma 3.4 we have $\mathrm{BWT}_{k} \equiv_{\mathrm{W}} \mathrm{RT}_{1, k}$, and hence the second claim follows from Proposition 7.3 .

Since $\mathrm{K}_{\mathbb{N}} \equiv_{\mathrm{W}} \mathrm{C}_{2}^{*}$ by [7, Proposition 10.9] we get the following conclusion.

Proposition 7.5 (Compact choice). $\mathrm{K}_{\mathbb{N}} \leq_{\mathrm{W}} \mathrm{SRT}_{1,+}$ and $\mathrm{K}_{\mathbb{N}} \not_{\mathrm{W}} \mathrm{RT}_{1, k}$ for all $k \geq 1$.

Proof. Since $\mathrm{C}_{2} \leq_{\mathrm{W}} \mathrm{SRT}_{1,2}$, we obtain $\mathrm{K}_{\mathbb{N}} \equiv_{\mathrm{W}} \mathrm{C}_{2}^{*} \leq_{\mathrm{W}} \mathrm{SRT}_{1,2}^{*} \leq_{\mathrm{W}} \mathrm{SRT}_{1,+}$ by Corollary 3.21. On the other hand, $\mathrm{C}_{k+1} \leq \leq_{\mathrm{W}} \mathrm{RT}_{1, k}$ and $\mathrm{C}_{k+1} \leq_{\mathrm{W}} \mathrm{K}_{\mathbb{N}}$ implies $\mathrm{K}_{\mathbb{N}} \leq \underline{\mathrm{W}}_{\mathrm{W}} \mathrm{RT}_{1, k}$ for all $k \geq 1$.

We obtain the following corollary.

Corollary 7.6 (Jump of compact choice). $\mathrm{K}_{\mathbb{N}}^{\prime} \equiv_{\mathrm{W}} \mathrm{RT}_{1, \mathbb{N}}$ and $\mathrm{K}_{\mathbb{N}}^{\prime} \underline{\mathrm{E}}_{\mathrm{W}} \mathrm{SRT}_{2,+}$.

Proof. $\mathrm{K}_{\mathbb{N}}^{\prime} \equiv_{\mathrm{W}} \mathrm{RT}_{1, \mathbb{N}}$ follows from Proposition 3.4 since $\mathrm{K}_{\mathbb{N}}^{\prime} \equiv_{\mathrm{sW}} \mathrm{BWT}_{\mathbb{N}}$. Corollary 6.6 implies $\mathrm{K}_{\mathbb{N}}^{\prime} \underline{E}_{\mathrm{W}} \mathrm{SRT}_{2, k}$ for all $k \geq 1$, and since $\mathrm{K}_{\mathbb{N}}^{\prime}$ is countably irreducible (as any jump is by [7, Proposition 5.8]) we obtain $\mathrm{K}_{\mathbb{N}}^{\prime} \not \mathbb{W}_{\mathrm{W}} \mathrm{SRT}_{2,+}$.

The equivalence $\mathrm{K}_{\mathbb{N}}^{\prime} \equiv_{\mathrm{W}} \mathrm{R} T_{1, \mathbb{N}}$ corresponds to a well-known theorem of Hirst [25], which says that $R T_{<\infty}^{1}$ is equivalent to $B \Sigma_{2}^{0}$ over $\mathrm{RCA}_{0}$ (see also [22, Theorem 6.81]), 
whereas the second equivalence $\mathrm{K}_{\mathbb{N}}^{\prime} \mathbb{Z}_{\mathrm{W}} \mathrm{SRT}_{2,+}$ shows that the reverse mathematics result [12] (see also [22, Theorem 6.82]) that $\mathrm{SRT}_{2,2}$ proves $\mathrm{RT}_{<\infty}^{1}$ over $\mathrm{RCA}_{0}$ cannot be proved uniformly (for instance the proof presented in [22, Theorem 6.82] contains a non-constructive case distinction).

Corollary 7.6 yields also one direction of the following corollary, and the other direction follows since $\mathrm{RT}_{1, \mathbb{N}} \leq \mathrm{W} \lim ^{\prime}$, but $\mathrm{SRT}_{2,2} \mathbb{Z W}_{\mathrm{W}} \lim ^{\prime}$ and $\mathrm{SRT}_{2, \mathbb{N}} \mathbb{Z}_{\mathrm{W}}$ lim $^{\prime}$ by Corollary 4.20

Corollary 7.7. $\left.\mathrm{RT}_{1, \mathbb{N}}\right|_{\mathrm{W}} \mathrm{SRT}_{2,2}$ and $\left.\mathrm{RT}_{1, \mathbb{N}}\right|_{\mathrm{W}} \mathrm{SRT}_{2,+}$.

We can conclude the following results on $\mathrm{C}_{\mathbb{N}}$ from earlier results.

Proposition 7.8 (Closed choice). $\mathrm{C}_{\mathbb{N}} \equiv_{\mathrm{W}} \mathrm{SRT}_{1, \mathbb{N}}$ and $\mathrm{C}_{\mathbb{N}} \not_{\mathrm{W}} \mathrm{RT}_{1,+}$.

Proof. The first statement follows from Proposition 3.4] since $\mathrm{C}_{\mathbb{N}} \equiv_{\mathrm{W}} \lim _{\mathbb{N}}$ by 7 , Proposition 3.8], and the second statement follows from the fact that $C_{\mathbb{N}}$ is countably irreducible by [7, Fact 3.2] (since every strong fractal is a fractal and hence countably irreducible), but $C_{\mathbb{N}} \leq_{W} \mathrm{RT}_{1, k}$ for some $k \geq 1$ is impossible by Proposition 7.5 since $\mathrm{K}_{\mathbb{N}} \leq_{\mathrm{W}} \mathrm{C}_{\mathbb{N}}$.

Altogether, we have justified the way choice problems are displayed in Figure 1. Finally, we provide the following reduction that can be derived from earlier results.

Theorem 7.9 (Jumps of compact choice). $\mathrm{K}_{\mathbb{N}}^{(n)} \leq{ }_{\mathrm{W}} \mathrm{SRT}_{n, \mathbb{N}}$ for all $n \geq 2$.

Proof. By Propositions 3.4 and 4.1 and since jumps are monotone with respect to $\leq_{\mathrm{sW}}$ we obtain $\mathrm{K}_{\mathbb{N}}^{(n)} \equiv_{\mathrm{sW}} \mathrm{BWT}_{\mathbb{N}}^{(n-1)} \leq_{\mathrm{sW}} \mathrm{CRT}_{1, \mathbb{N}}^{(n-1)} \leq_{\mathrm{sW}} \mathrm{CSRT}_{n, \mathbb{N}} \leq_{\mathrm{W}} \mathrm{SRT}_{n, \mathbb{N}}$.

The special case for $n=2$ can be seen as the uniform version of a theorem of Cholak, Jockusch and Slaman [12, see also [22, Theorem 6.89], which states that $\mathrm{SRT}_{<\infty}^{2}$ proves $\mathrm{B} \Sigma_{3}^{0}$ over $\mathrm{RCA}_{0}$. In light of Corollary 7.6 and Theorem 7.9 there is quite some gap in between $\mathrm{SRT}_{2,+}$ and $\mathrm{SRT}_{2, \mathbb{N}}$. The diagram summarizes our calibration of choice problems by Ramsey's theorem.

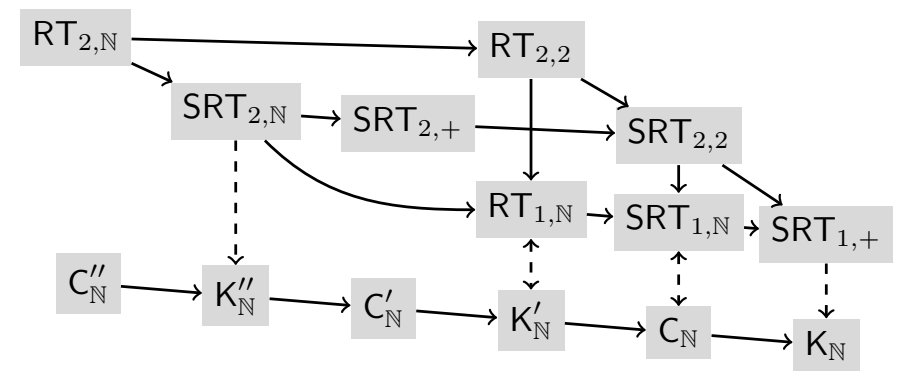

FiguRE 5. Closed and compact choice on natural numbers calibrated with Ramsey's theorem in the Weihrauch lattice.

Further questions could be studied along these lines. We mention the following question. The first part of this question is related to [22, Theorem 6.85] and the second part to [22, Open Question 6.92].

Question 7.10 (Jump of closed choice). Does $C_{\mathbb{N}}^{\prime} \leq_{W} R T_{2,2}$ hold? Does $C_{\mathbb{N}}^{\prime \prime} \leq_{W} R T_{2, \mathbb{N}}$ hold?

In light of [22, Theorem 6.85] a positive answer to the first part of this question seems unlikely. Another natural question is how cluster point problems are related to Ramsey's theorem. We can say at least something.

Corollary 7.11 (Cluster point problem). $\mathrm{CL}_{\mathbb{R}} \leq_{\mathrm{W}} \mathrm{RT}_{3,3}$. 
Proof. We recall that $\mathrm{CL}_{\mathbb{N}} \equiv_{\mathrm{W}} \mathrm{C}_{\mathbb{N}}^{\prime}$ and $\mathrm{CL}_{2^{\mathbb{N}}} \equiv_{\mathrm{W}} \mathrm{C}_{2^{\mathbb{N}}}^{\prime} \equiv_{\mathrm{W}} \mathrm{WKL}^{\prime}$ by $[7$, Theorem 9.4]. By [7, Proposition 9.15] we have $\mathrm{CL}_{\mathbb{R}} \equiv_{\mathrm{W}} \mathrm{C}_{\mathbb{N}}^{\prime} \times \mathrm{CL}_{2^{\mathbb{N}}} \equiv_{\mathrm{W}} \mathrm{C}_{\mathbb{N}}^{\prime} \times \mathrm{WKL}^{\prime}$. Hence, we obtain with Proposition 7.2, Theorem 7.9 and Corollary 3.30

$$
\mathrm{CL}_{\mathbb{R}} \equiv_{\mathrm{W}} \mathrm{C}_{\mathbb{N}}^{\prime} \times \mathrm{WKL}^{\prime} \leq_{\mathrm{W}} \mathrm{K}_{\mathbb{N}}^{\prime \prime} \times \mathrm{WKL}^{\prime} \leq_{\mathrm{W}} \mathrm{RT}_{2, \mathbb{N}} \times \mathrm{RT}_{3,2} \leq_{\mathrm{W}} \mathrm{RT}_{3,3}
$$

The last mentioned reduction holds by Theorem 3.24 .

However, it is not immediately clear whether the following holds.

Question 7.12. $\mathrm{CL}_{\mathbb{R}} \leq_{\mathrm{W}} \mathrm{RT}_{3,2}$ ?

\section{Conclusion}

We have studied the uniform computational content of Ramsey's theorem in the Weihrauch lattice, and we have clarified many aspects of Ramsey's theorem in this context. Key results are the lower bound provided in Theorem 3.5, the theorems on products (Theorem 3.24) and parallelization (Theorem 3.28), as well as the theorem on jumps (Theorem 4.3) and the upper bounds in Corollary 4.15]derived from it. From this tool box of key results (together with the squashing theorem, Theorem 1.7) we were able to derive a number of interesting consequences, such as the characterization of the parallelization of Ramsey's theorem in Corollary 4.18] and the effect of increasing numbers of colors in Theorem 4.22. The separation tools provided in Section 6 have led to some further clarity. A number of important questions regarding the uniform behavior of Ramsey's theorem were left open. Hopefully, some future study will shed further light on this question.

\section{ACKnowledgments}

We would like to thank Steve Simpson and Ludovic Patey for helpful comments on an earlier version of this article and the anonymous referee for his or her very careful proof reading that helped us to improve the presentation of the article.

\section{REFERENCES}

[1] Stefano Berardi and Silvia Steila. Ramsey Theorem for Pairs As a Classical Principle in Intuitionistic Arithmetic. In Ralph Matthes and Aleksy Schubert, editors, 19th International Conference on Types for Proofs and Programs (TYPES 2013), volume 26 of Leibniz International Proceedings in Informatics (LIPIcs), pages 64-83, Dagstuhl, Germany, 2014. Schloss Dagstuhl-Leibniz-Zentrum fuer Informatik.

[2] Vasco Brattka. Effective Borel measurability and reducibility of functions. Mathematical Logic Quarterly, 51(1):19-44, 2005.

[3] Vasco Brattka, Matthew de Brecht, and Arno Pauly. Closed choice and a uniform low basis theorem. Annals of Pure and Applied Logic, 163:986-1008, 2012.

[4] Vasco Brattka and Guido Gherardi. Effective choice and boundedness principles in computable analysis. The Bulletin of Symbolic Logic, 17(1):73-117, 2011.

[5] Vasco Brattka and Guido Gherardi. Weihrauch degrees, omniscience principles and weak computability. The Journal of Symbolic Logic, 76(1):143-176, 2011.

[6] Vasco Brattka, Guido Gherardi, and Rupert Hölzl. Probabilistic computability and choice. Information and Computation, 242:249-286, 2015.

[7] Vasco Brattka, Guido Gherardi, and Alberto Marcone. The Bolzano-Weierstrass theorem is the jump of weak König's lemma. Annals of Pure and Applied Logic, 163:623-655, 2012.

[8] Vasco Brattka, Matthew Hendtlass, and Alexander P. Kreuzer. On the uniform computational content of computability theory. arXiv 1501.00433, 2015.

[9] Vasco Brattka, Peter Hertling, and Klaus Weihrauch. A tutorial on computable analysis. In S. Barry Cooper, Benedikt Löwe, and Andrea Sorbi, editors, New Computational Paradigms: Changing Conceptions of What is Computable, pages 425-491. Springer, New York, 2008.

[10] Vasco Brattka, Stéphane Le Roux, Joseph S. Miller, and Arno Pauly. Connected choice and the Brouwer fixed point theorem. arXiv 1206.4809, 2016.

[11] Vasco Brattka and Arno Pauly. On the algebraic structure of Weihrauch degrees. arXiv 1604.08348, 2016. 
[12] Peter A. Cholak, Carl G. Jockusch, and Theodore A. Slaman. On the strength of Ramsey's theorem for pairs. Journal of Symbolic Logic, 66(1):1-55, 2001.

[13] Peter A. Cholak, Carl G. Jockusch, Jr., and Theodore A. Slaman. Corrigendum to: "On the strength of Ramsey's theorem for pairs". Journal of Symbolic Logic, 74(4):1438-1439, 2009.

[14] Chi Tat Chong, Steffen Lempp, and Yue Yang. On the role of the collection principle for $\Sigma_{2}^{0}$ formulas in second-order reverse mathematics. Proceedings of the American Mathematical Society, 138(3):1093-1100, 2010.

[15] Chi Tat Chong, Theodore A. Slaman, and Yue Yang. The metamathematics of stable Ramsey's theorem for pairs. Journal of the American Mathematical Society, 27(3):863-892, 2014.

[16] François G. Dorais, Damir D. Dzhafarov, Jeffry L. Hirst, Joseph R. Mileti, and Paul Shafer. On uniform relationships between combinatorial problems. Transactions of the American Mathematical Society, 368(2):1321-1359, 2016.

[17] Damir D. Dzhafarov. Cohesive avoidance and strong reductions. Proceedings of the American Mathematical Society, 143(2):869-876, 2015.

[18] Damir D. Dzhafarov. Strong reductions between combinatorial principles. Journal of Symbolic Logic, 81(4):1405-1431, 2016.

[19] Paul Erdős, András Hajnal, Attila Máté, and Richard Rado. Combinatorial set theory: partition relations for cardinals, volume 106 of Studies in Logic and the Foundations of Mathematics. North-Holland Publishing Co., Amsterdam, 1984.

[20] Guido Gherardi and Alberto Marcone. How incomputable is the separable Hahn-Banach theorem? Notre Dame Journal of Formal Logic, 50(4):393-425, 2009.

[21] Petr Hájek and Pavel Pudlák. Metamathematics of first-order arithmetic. Perspectives in Mathematical Logic. Springer-Verlag, Berlin, 1993.

[22] Denis R. Hirschfeldt. Slicing the Truth, On the Computable and Reverse Mathematics of Combinatorial Principles, volume 28 of Lecture Notes Series, Institute for Mathematical Sciences, National University of Singapore. World Scientific, Singapore, 2015.

[23] Denis R. Hirschfeldt and Carl G. Jockusch. On notions of computability-theoretic reduction between $\Pi_{2}^{1}$ principles. Journal of Mathematical Logic, 16(1):1650002, 59, 2016.

[24] Denis R. Hirschfeldt, Carl G. Jockusch, Jr., Bjørn Kjos-Hanssen, Steffen Lempp, and Theodore A. Slaman. The strength of some combinatorial principles related to Ramsey's theorem for pairs. In Computational prospects of infinity. Part II. Presented talks, volume 15 of Lect. Notes Ser. Inst. Math. Sci. Natl. Univ. Singap., pages 143-161. World Sci. Publ., Hackensack, NJ, 2008.

[25] Jeff L. Hirst. Combinatorics in Subsystems of Second Order Arithmetic. Phd thesis, The Pennsylvania State University, State College, USA, 1987.

[26] Carl G. Jockusch, Jr. Ramsey's theorem and recursion theory. The Journal of Symbolic Logic, 37:268-280, 1972.

[27] Alexander S. Kechris. Classical Descriptive Set Theory, volume 156 of Graduate Texts in Mathematics. Springer, Berlin, 1995.

[28] Alexander Kreuzer and Ulrich Kohlenbach. Ramsey's theorem for pairs and provably recursive functions. Notre Dame Journal of Formal Logic, 50(4):427-444 (2010), 2009.

[29] Alexander P. Kreuzer and Ulrich Kohlenbach. Term extraction and Ramsey's theorem for pairs. Journal of Symbolic Logic, 77(3):853-895, 2012.

[30] Jiayi Liu. RT 2 does not imply WKL $\mathrm{WL}_{0}$ Journal of Symbolic Logic, 77(2):609-620, 2012.

[31] Lu Liu. Cone avoiding closed sets. Transactions of the American Mathematical Society, 367(3):1609-1630, 2015.

[32] Ludovic Patey. The weakness of being cohesive, thin or free in reverse mathematics. Israel Journal of Mathematics, 216:905-955, 2016.

[33] Arno Pauly. How incomputable is finding Nash equilibria? Journal of Universal Computer Science, 16(18):2686-2710, 2010.

[34] Tahina Rakotoniaina. On the Computational Strength of Ramsey's Theorem. PhD thesis, Department of Mathematics and Applied Mathematics, University of Cape Town, Rondebosch, South Africa, 2015.

[35] Frank P. Ramsey. On a Problem of Formal Logic. Proceedings of the London Mathematical Society, S2-30(1):264, 1930.

[36] David Seetapun and Theodore A. Slaman. On the strength of Ramsey's theorem. Notre Dame Journal of Formal Logic, 36(4):570-582, 1995. Special Issue: Models of arithmetic.

[37] Stephen G. Simpson. Subsystems of Second Order Arithmetic. Perspectives in Logic, Association for Symbolic Logic. Cambridge University Press, Poughkeepsie, second edition, 2009.

[38] Ernst Specker. Ramsey's theorem does not hold in recursive set theory. In Logic Colloquium '69 (Proc. Summer School and Colloq., Manchester, 1969), pages 439-442. North-Holland, Amsterdam, 1971.

[39] Klaus Weihrauch. Computable Analysis. Springer, Berlin, 2000. 
Faculty of Computer Science, Universität der Bundeswehr München, Germany and Department of Mathematics \& Applied Mathematics, University of Cape Town, South AFRICA 9

E-mail address: Vasco.Brattka@cca-net.de

Department of Mathematics \& Applied Mathematics, University of Cape Town, South AFRICA 10

E-mail address: fenoira@gmail.com

${ }^{9}$ The research leading to these results has received funding from the National Research Foundation of South Africa and by the People Programme (Marie Curie Actions) of the European Union's Seventh Framework Programme FP7/2007-2013/ under REA grant agreement no PIRSES-GA-2011-294962COMPUTAL.

${ }^{10}$ Tahina Rakotoniaina has additionally been supported by the German Academic Exchange Service (DAAD). 